\title{
On the Liouvillian solutions to the perturbation equations of the Schwarzschild black hole
}

\author{
Evangelos Melas ${ }^{1}$
}

(Dated: September 20, 2018)

It is well known that the equations governing the evolution of scalar, electromagnetic and gravitational perturbations of the background geometry of a Schwarzschild black hole can be reduced to a single master equation. We use Kovacic's algorithm to obtain all Liouvillian solutions, i.e., essentially all solutions in terms of quadratures, of this master equation. We show that all solutions in quadratures of this equation contain a polynomial solution to an associated ordinary differential equation (ODE). In the case of the gravitational perturbations only, for a Liouvillian solution $\chi$ to the master equation, this ODE reduces to an algebraic equation of the first order. In all the other cases, apart from a few trivial cases which do not give any Liouvillian solutions, this ODE falls into the confluent Heun class. In the case of the gravitational perturbations, for the Liouvillian solution $\chi \int \frac{\mathrm{d} r_{*}}{\chi^{2}}$, we find in "closed form" the polynomial solution $\mathrm{P}$ to the associated confluent Heun ODE. We prove that the Liouvillian solution $\chi \int \frac{\mathrm{d} r_{*}}{\chi^{2}}$ is a product of elementary functions, one of them being the polynomial P. We extend previous results by Hautot and use the extended results we derive in order to prove that $\mathrm{P}$ admits a finite expansion in terms of truncated confluent hypergeometric functions of the first kind. We also prove, by using the extended results we derive, that $\mathrm{P}$ admits also a finite expansion in terms of associated Laguerre polynomials. Remarkably both expansions entail not constant coefficients but appropriate function coefficients instead. We highlight the relation of these results with inspiring new developments. We prove, save for two unresolved cases, that the Liouvillian solutions $\chi$ and $\chi \int \frac{\mathrm{d} r_{*}}{\chi^{2}}$, initially found by Chandrasekhar, are the only Liouvillian solutions to the master equation. We improve previous results in the literature on this problem and compare our results with them. Comments are made for a more efficient implementation of Kovacic's algorithm to any second order ODE with rational function coefficients. Our results set the stage for deriving similar results in other black hole geometries 4-dim and higher. 


\section{INTRODUCTION}

It is well known (see for instance ${ }^{\underline{1}}$ ) that the equations governing the evolution of external sourceless test fields (be it scalar, electromagnetic, or a perturbation of the gravitational field itself) on the background geometry of a Schwarzschild black hole can be reduced to a single second order ordinary differential equation (ODE) with rational function coefficients. This master equation, equation (6), assumes formally the Schrödinger form, with an effective curvature potential. The functional form of this potential is such that it is difficult, in general, to obtain exact solutions to the master equation. For that reason, most of the work done so far has used approximation methods.

For instance, in the gravitational case, the master equation is the Regge-Wheeler equation $\underline{\underline{2}} \underline{\underline{4}}$ (RWE), and a number of numerical, semianalytic and analytic approximation techniques have been applied over the years for calculating frequencies of the quasinormal modes of Schwarzschild black holes (see for example $e^{-\underline{11}}$ and references therein). The quasinormal modes are solutions of the Regge-Wheeler and Zerilli equations $12, \underline{13}$ (both to be discussed in section 2) that fulfil the boundary conditions: the waves must be outgoing at spatial infinity and ingoing across the event horizon.

However, Chandrasekhar ${ }^{\underline{14}}$ found solutions in terms of quadratures for algebraically special perturbations of the Schwarzschild, Reissner-Nordström and Kerr black holes. These algebraically special perturbations excite gravitational waves that are either purely ingoing or purely outgoing. Such algebraically special perturbations of the Kerr space-time were first considered in passing by Wald $\underset{15}{ }$, who showed that necessary and sufficient condition for the existence of these algebraically special perturbations is the vanishing of the Starobinsky constant, though the explicit solutions in quadratures of the perturbation equations were not given by him. Wald's result generalized an earlier one by Couch \& Newman $\underline{16}$ in the context of the Schwarzschild black hole.

The important point to be noted here is that these algebraically special perturbations solutions can occur only for special values of the frequency of the perturbations as first noticed by Couch \& Newman $\underline{16}$ in the context of the Schwarzschild black hole. The vanishing of the Starobinsky constant, first noticed by Wald $\frac{15}{5}$ in the case of the Kerr black hole, and then studied by Chandrasekhar $\underline{\underline{14}}$ in the case of Kerr, Reissner-Nordström, and Schwarzschild black holes, is precisely the condition that the algebraically special perturbations occur only 
for special values of the frequencies in all black hole geometries. In the Schwarzschild case, Qi and Schutz $\frac{17}{}$ have shown that the algebraically special solution is the linear approximation to the Robinson-Trautman solution, when that solution tends to the Schwarzschild solution.

In this paper we reexamine the issue of obtaining all solutions in quadratures of the perturbation equations of the Schwarzshild geometry by using Kovacic's algorithm $\underline{18-24}$. The employment of Kovacic's algorithm to find all possible Liouvillian solutions of the master equation which governs the evolution of the perturbations of the Schwarzshild geometry was initiated in $\frac{25}{}$ and the only published work on the subject is, to the best of our knowledge ${ }^{26}$, by Couch \& Holder (2007; henceforth C.H.).

In our work we address problems unnoticed in C.H. and in related work ${ }^{27}-\underline{40}$. These problems are described in subsections $1 \mathrm{IB}$, IC, and ID. In a nutshell, these problems are:

1. The search for pairs of Liouvillian solutions $\eta$ and $\eta \int \frac{e^{-\int a}}{\eta^{2}}$ in Kovacic's algorithm.

2. The expression of the second Liouvillian solution $\eta \int \frac{e^{-\int a}}{\eta^{2}}$, initially found by Chandrasekhar $\stackrel{14}{ }$, in the case of the gravitational perturbations of the Schwarzschild geometry, as a product of elementary functions, one of them being a polynomial $\Pi$, which is a solution to an associated confluent Heun equation.

3. The determination of $\Pi$ in "closed-form".

4. The expansion of $\Pi$ in terms of confluent hypergeometric functions of the first kind.

5. The expansion of $\Pi$ in terms of associated Laguerre polynomials.

We comment on problem 1 in subsection IB and solve it in section V] We comment on problems 2 and 3 in subsection [C] and solve them in section $\mathrm{V}$. We comment on problems 4 and 5 in subsection [D and solve them in section VII (Theorem 4 and Theorem 5).

Our results are apt to generalization to other black hole geometries, 4-dim and higher $\stackrel{41}{ }$.

\section{A. Kovacic's algorithm}

Kovacic's algorithm will find all possible "closed-form" solutions (i.e., essentially, all solutions in terms of quadratures) of linear second order homogeneous ODEs with complex 
rational function coefficients of the form

$$
y^{\prime \prime}+a y^{\prime}+b y=0
$$

where $a$ and $b$ are rational functions of a complex variable $x$. The "closed-form" solution means a Liouvillian solution, i.e. one that can be expressed in terms of algebraic functions, exponentials and indefinite integrals. Let $\eta$ be a (non-zero) Liouvillian solution of the differential equation (11). The method of reduction of order produces a second Liouvillian solution, namely

$$
\eta \int \frac{e^{-\int a}}{\eta^{2}}
$$

Kovacic's algorithm reduces the problem of finding Liouvillian solutions to equation (1) to the problem of finding polynomial solutions to an associated set $\mathcal{D}$ of linear ODEs whose coefficients are rational functions over the field of complex numbers. The algorithm is so constructed that if no polynomial solutions exist to any of the differential equations in $\mathcal{D}$ then equation (11) has no liouvillian solutions, whereas, if a polynomial solution exists to a differential equation in $\mathcal{D}$ then this polynomial solution will give rise to a Liouvillian solution to equation (1), and, different polynomial solutions to the differential equations in $\mathcal{D}$ give rise to different Liouvillian solutions to equation (1).

The most difficult aspects of applying Kovacic's algorithm are reduced to the solution of the following three problems:

1. In general, it is not true, that if the polynomial solution to one of the differential equations in $\mathcal{D}$ gives rise to a Liouvillian solution $\eta$ to equation (1) then the polynomial solution to another differential equation in $\mathcal{D}$ will give rise to the second solution $\eta \int \frac{e^{-\int a}}{\eta^{2}}$ of equation (11), which is also Liouvillian. This is precisely the case in the problem we are examining here; it turns out, as we show in subsection $\mathrm{VB}$, that in the case of the gravitational perturbations of the Schwarzschild geometry there exists Liouvillian solution $\eta$, and it takes us considerable effort in subsection $\mathrm{VC}$ to prove that Kovacic's algorithm does also give rise to the second Liouvillian solution $\eta \int \frac{e^{-\int a}}{\eta^{2}}$.

2. In general, it is not easy, to find polynomial solutions, if they exist at all, to the differential equations in $\mathcal{D}$. This difficulty becomes more apparent when the degree of the sought polynomial solution is not fixed but it depends on a parameter which appears in the coefficients of the differential equation in $\mathcal{D}$. When a differential equation in $\mathcal{D}$ 
leads to a 2 -term recurrence relation for the coefficients of its Frobenius solutions the problem of finding a polynomial solution to it can be solved in a systematic way, either its degree is fixed or depends on a parameter which appears in the coefficients of the differential equation in $\mathcal{D}$. However, the difficulty becomes even more prominent when the differential equation in $\mathcal{D}$ leads to a $d$-term recurrence relation, $d=3,4,5, \ldots$, for the coefficients of its Frobenius solutions, since in general, $d$-term recurrences do not admit "closed-form" solutions. This is precisely the case in the problem we are examining here; as we show in subsection $\mathrm{VC}$, one of the differential equations in $\mathcal{D}$ associated to equation (1), in the case of the gravitational perturbations of the Schwarzschild geometry, is equation (87) which falls into the confluent Heun class, and as such, leads to a 3 -term recurrence relation for the coefficients of its Frobenius solutions. Moreover the degree of the sought polynomial solution to equation (87) is not fixed but it depends on a parameter which appears in the coefficients of (87). It is the structure of Kovacic's algorithm in combination with the method we follow in subsection $\mathrm{VC}$ which allow us to find in "closed-form" a polynomial solution to equation (87).

3. As we said Kovacic's algorithm reduces the problem of finding Liouvillian solutions to equation (11) to the problem of finding polynomial solutions to an associated set $\mathcal{D}$ of linear ODEs. Let $\mathcal{M}$ be a member of $\mathcal{D}$. Kovacic's algorithm determines the degree $d$ of a polynomial solution to $\mathcal{M}$, if such a polynomial solution exists. The degree $d$ can be easily determined independently of the determination of $d$ by Kovacic's algorithm. For each $\mathcal{M}$ the following problem must be solved:

Find all polynomial solutions of degree $d$ to $\mathcal{M}$, or else, prove that $\mathcal{M}$ does not admit a polynomial solution.

The difficulty of the problem of finding all polynomial solutions of degree $d$ to $\mathcal{M}$ constitutes the second difficult aspect of applying Kovacic's algorithm. Here we restrict attention to the problem of proving that $\mathcal{M}$ does not admit polynomial solution, when this is the case. We note that when $\mathcal{M}$ leads to a 2 -term recurrence relation for the coefficients of its Frobenius series solutions the problem of proving that the Frobenius series does not terminate, when this is the case, can be solved in a systematic way, either its degree is fixed or it depends on a parameter which appears in the coefficients 
of $\mathcal{M}$. However when $\mathcal{M}$ leads to a $q$-term recurrence relation, $q=3,4,5, \ldots$, for the coefficients of its Frobenius series solutions the problem of proving that the Frobenius series does not terminate, is by no means a trivial problem and a variety of approaches can be employed to solve the problem. These methods, in the case $q=3$, are elaborated in subsection VIIID,

Problems 1 and 2 are respectively problems 1 and 3 in the list of problems in section [1. C.H. $\stackrel{26}{a}$ address and solve partially only problem 3 . We address all problems 1,2 , and 3 . We comment on problem 1 in subsection $\llbracket \mathrm{B}$ and solve it in section $\mathrm{V}$. We comment on problem 2 in subsection $[\mathrm{IC}$ and solve it in section $\mathrm{V}$. We solve partially problem 3 in sections $\nabla$ and VIII.

We improve the results derived by C.H. $\frac{26}{2}$ on the solution of problem 3. Namely, the results derived in subsections VIIIA, VIIIB, VIIIC, VIIID, VIIIE, and VIIIG are new. We compare our results on the solution of problem 3 with the results of C.H. on the solution of problem 3 in subsection VIIIF. This is as far as the application of Kovacic's algorithm is concerned.

In subsection $\mathrm{VD}$ we prove that the second Liouvillian solution $\eta \int \frac{e^{-\int a}}{\eta^{2}}$, initially found by Chandrasekhar $\underline{\underline{14}}$, in the case of the gravitational perturbations of the Schwarzschild geometry, can be expressed as a product of elementary functions, one of these elementary functions being a polynomial solution to a confluent Heun equation.

Moreover, we address and solve a problem which is not addressed at all by C.H. $\underline{\underline{26}}$; this is the union of problems 4 and 5 in the list of problems in section [1. Motivated by previous work on the confluent Heun equation and the previous knowledge and experience acquired from non-relativistic quantum mechanics $\frac{42}{}$, we expect that this polynomial can be expressed as a linear combination of (confluent) hypergeometric functions.

We comment on this problem in subsection ID and solve it in section VII, where we prove that the polynomial in question can be expressed as a sum, with appropriate function coefficients, of truncated confluent hypergeometric functions of the first kind (Theorem 4). Furthermore we prove that the polynomial in question can be expressed as a sum, with appropriate function coefficients, of associated Laguerre polynomials (Theorem 5). 


\section{B. The search for pairs $\eta$ and $\eta \int \frac{e^{-\int a}}{\eta^{2}}$}

It is not generally true ${ }^{20}$ that if the polynomial solution to one of the differential equations in $\mathcal{D}$ gives rise to a Liouvillian solution $\eta$ to equation (11) then the polynomial solution to another differential equation in $\mathcal{D}$ will give rise to $\eta \int \frac{e^{-\int a}}{\eta^{2}}$. In fact establishing when this holds, as we said in subsection IA, is one of the most difficult aspects of applying Kovacic's algorithm and it can take considerable amount of time and effort to find the pair(s) of equations in $\mathcal{D}$, if they exist at all, which yield the Liouvillian solutions $\eta$ and $\eta \int \frac{e^{-\int a}}{\eta^{2}}$ to equation (11).

C.H. do not even mention the possibility that Kovacic's algorithm, besides the Liouvillian solution $\eta$ may also gives rise to the Liouvillian solution $\eta \int \frac{e^{-\int a}}{\eta^{2}}$. In fact in section $\mathrm{V}$ we prove that this is indeed the case for the gravitational perturbations of the Schwarzschild geometry. In particular in subsection $\mathrm{VB}$ we prove that Kovacic's algorithm gives rise to the algebraically special Liouvillian perturbation $\eta$ of Schwarzschild black hole, found by Chandrasekhar ${ }^{14}$, which excites gravitational waves that are either purely ingoing or purely outgoing.

This is the easy part of the proof and has been given in a slightly different form by C.H.. We include it here because it is short and for completeness of the presentation. However, the difficult part of the proof in section $\mathrm{V}$, not given by C.H., is given in subsection V $\mathrm{C}$, In subsection $\mathrm{VC}$ we prove that Kovacic's algorithm, in the case of the gravitational perturbations of the Schwarzschild geometry, gives also rise to the Liouvillian solution $\eta \int \frac{e^{-\int a}}{\eta^{2}}$.

Both algebraically special Liouvillian solutions $\eta$ and $\eta \int \frac{e^{-\int a}}{\eta^{2}}$ to the RWE equation were found by Chandrasekhar $\underline{14}$. However this does not render subsection VB superfluous. The significance of this subsection does not result from the discovery of the second Liouvillian solution $\eta \int \frac{e^{-\int a}}{\eta^{2}}$ but rather from the proof itself that Kovacic's algorithm gives also rise to the second Liouvillian solution $\eta \int \frac{e^{-\int a}}{\eta^{2}}$ and from other results which are obtained in the course of the proof.

In particular, in subsections $\mathrm{VC}$, $\mathrm{VD}$, we prove that $\eta \int \frac{e^{-\int a}}{\eta^{2}}$ can be expressed via elementary functions, one of them being a polynomial solution to a confluent Heun equation. This result remained unnoticed by Chandrasekhar $\stackrel{14}{ }$. The proof in subsection $\mathrm{VC}$ is also an indispensable part of the proof that there are no other Liouvillian solutions to the master equation (6) apart from those found by Chandrasekhar $\underline{14}$. 
We note in passing that it has been erroneously claimed ${ }^{\underline{43}}$ that Chandrasekhar found $\underline{14}$ only the Liouvillian solution $\eta \int \frac{e^{-\int a}}{\eta^{2}}$ and that he missed the other Liouvillian solution $\eta$. A careful reading of Chandrasekhar's paper $\underline{\underline{14}}$ proves that this is false.

Most likely the proof in subsection $\mathrm{VC}$ that Kovacic's algorithm gives also rise to the Liouvillian solution $\eta \int \frac{e^{-\int a}}{\eta^{2}}$ is going to be useful to future applications of the algorithm. Indeed, the logical structure of the proof does not depend on the particular problem at hand, namely the linear perturbations of the Schwarzschild geometry, but it is tailored for identifying Liouvillian pairs $\eta$ and $\eta \int \frac{e^{-\int a}}{\eta^{2}}$ when the order of one of the polynomial solutions associated to the Liouvillian solutions $\eta, \eta \int \frac{e^{-\int a}}{\eta^{2}}$ is not fixed but it depends on a parameter which appears in the coefficients of the polynomial solution.

This situation appears quite frequently in many problems of mathematical physics and in particular on the perturbation theory of the black-hole geometries in 4-dim and higher where this parameter comes from the master equation which governs the evolution of the perturbations. As the relevant analysis in section $\Pi$ shows this parameter is the frequency of the monochromatic waves to which the perturbation is Fourier decomposed. Therefore it is highly likely that analogues and/or generalizations of the proof in subsection $\mathrm{VC}$ are going to be useful in the search for pairs of Liouvillian solutions $\eta$ and $\eta \int \frac{e^{-\int a}}{\eta^{2}}$ in subsequent applications of the algorithm; either these applications refer to black hole geometries, or otherwise.

The absence of search for pairs $\eta$ and $\eta \int \frac{e^{-\int a}}{\eta^{2}}$, which is one of the most difficult aspects of applying Kovacic's algorithm is not unique with C.H., but it is also observed in other applications of Kovacic's algorithm (e.g. $\underline{\underline{40}}$ ). The cause of this negligence can be traced back to the original article by Kovacic $\underline{\underline{18}}$ in which no mention is made of the efficacy of the algorithm to capture the second Liouvillian solution $\eta \int \frac{e^{-\int a}}{\eta^{2}}$ when a Liouvillian solution $\eta$ is identified.

Subsequent works on Kovacic's algorithm $\underline{\underline{19}} \underline{\underline{24}}$ remain insistently silent on this point; the only exception being $\underline{20}$ where on page we 214 we read: "When one liouvillian solution is found, another one, linearly independent, is $\eta \int \frac{e^{-\int a}}{\eta^{2}}$. This second solution is not always detected by the algorithm;". This scarce and inconclusive mention in the literature on the existence of pairs $\eta$ and $\eta \int \frac{e^{-\int a}}{\eta^{2}}$ in the Liouvillian solutions found by Kovacic's algorithm probably explains the lack of search for such pairs in the applications and extensions of the algorithm so far $\underline{18} \underline{2}, \underline{26}, 40$. 


\section{Liouvillian solutions, confluent Heun equation and its polynomial solutions}

In subsections $\mathrm{VB}$ and $\mathrm{VC}$ we prove that RWE equation, which governs the gravitational perturbations of the Schwarzschild geometry, admits two Liouvillian solutions, $\eta$ and $\eta \int \frac{e^{-\int a}}{\eta^{2}}$, initially found by Chandrasekhar $\underline{14}$.

However, Chandrasekhar did not obtain the results of subsection $\mathrm{VC}$ and $\mathrm{VD}$; As we prove in subsection $\overline{\mathrm{VC}}$, the Liouvillian solution $\eta \int \frac{e^{-\int a}}{\eta^{2}}$, initially found by Chandrasekhar $\underline{\underline{14}}$, is the product of functions (equation (93)), one of them being a polynomial solution to an associated confluent Heun equation, equation (87); equation (87) falls into the confluent Heun class after a trivial change of the independent variable, however, this does not affect in any way the results and conclusions stated here.

An immediate corollary of this, as we show in subsection $\overline{\mathrm{VD}}$, is that $\eta \int \frac{e^{-\int a}}{\eta^{2}}$ is a product of elementary functions. This result, was given for the first time by Araujo and MacCallum ${ }^{\underline{44}}$ in an unpublished work; however, these authors do not give the proof, which we give in subsection $\overline{\mathrm{VC}}$, which leads to it.

It is difficult to imagine how we could have obtained the results of subsections $\mathrm{VC}$ and VD without applying Kovacic's algorithm to the master equation (6), RWE equation is a special case of (6) : It is the structure of Kovacic's algorithm in combination with the method we follow in subsection $\mathrm{VC}$ which allow to find in "closed-form" the polynomial factor in $\eta \int \frac{e^{-\int a}}{\eta^{2}}$.

Remarkably, we obtain in "closed-form" the polynomial factor in $\eta \int \frac{e^{-\int a}}{\eta^{2}}$, despite the fact that the associated confluent Heun equation (87), leads to a 3-term recurrence relation for the coefficients of its Frobenius solutions, and in general, 3-term recurrences do not admit "closed-form" solutions.

Regarding the associated confluent Heun equation (87) we note the following: The degree of the sought polynomial solution to equation (87), if such a polynomial solution exists at all, is found either by using Kovacic's algorithm or by a straightforward calculation. The crucial feature of the sought polynomial solution to equation (87) is that its degree is not fixed but it depends on a parameter which appears in the coefficients of (87).

It is precisely this feature which renders difficult to find polynomial solutions to (87) if we proceed with brutal force with any of the textbook methods, eg., by solving the associated linear homogeneous system, by considering the continued fraction associated to the 3 -term 
recurrence relation $\underline{\underline{45}}$ to which the confluent Heun equation leads, or by applying the relevant knowledge and experience from non-relativistic quantum mechanics $\underline{42}$ in relation with the hypergeometric function solutions to 3 -term recurrences arising from the time-independent Schrödinger equation.

The difficulties persist even if we apply not textbook methods but results and methods which have appeared in the relevant research on the subject, e.g., a new method of solving linear differential equations $\frac{46}{}$, of arbitrary order, and the subsequent technique to find polynomial solutions to linear differential equations $\underline{47}$, or, expansion of the solution of (87) in terms of confluent hypergeometric functions and the like $\underline{\underline{48}}, \underline{49}$.

Since previous experience and knowledge and other results on the subject do not offer any clues for finding polynomial solutions to equation (87), we follow instead another method in subsection $\mathrm{VC}$ which allows us to find a polynomial solution to equation (87) and at the same time to prove that Kovacic's algorithm does give rise to a pair of Liouvillian solutions $\eta$ and $\eta \int \frac{e^{-\int a}}{\eta^{2}}$.

The results in subsections $\mathrm{VB}, \mathrm{VC}$, and VIII, show that all Liouvillian solutions to the master equation (6) contain a polynomial; in all cases, this polynomial is solution to an associated confluent Heun differential equation $\underline{\underline{50}} \underline{\underline{56}}$. The only exception is studied in subsection $\nabla B$ and occurs in the case of the gravitational perturbations of the Schwarzschild geometry. In this case we do find a Liouvillian solution $\chi$, given in equation (85), to the master equation (6), and the polynomial which is contained in $\chi$ is of first order and it satisfies an ODE of second order, which reduces to an algebraic equation of first order for the constant term of the polynomial.

A precursor of this result appeared in $\underline{57-59}$ where it was shown that the master equation which governs the evolution of various perturbations on a class of black hole geometries falls into the Heun class after a change of the independent variable and after a subsequent change of the dependent variable. However a systematic, exhaustive construction of all Liouvillian solutions of the master equation (6) which governs the evolution of perturbations of the Schwarzschild geometry is out of the reach of these studies, this can only be accomplished by using Kovacic's algorithm. 


\section{Liouvillian solutions, truncated hypergeometric functions, and associated Laguerre polynomials}

The master equation (6), after the change of the dependent variable (8), becomes a time-independent Schrödinger type equation (equation (9)) with an effective curvature potential. For many potentials, e.g. for the harmonic oscillator potential, the Kratzer potential, the Morse potential, the Bargmann potential, the Pöschl-Teller potential, to mention a few, time-independent Schrödinger equation admits Liouvillian solutions in terms of truncated (confluent) hypergeometric functions $\stackrel{42}{ }$.

This suggests, given the general form of the Liouvillian solution obtained by applying Kovacic's algorithm to the master equation (6) (see e.g. equation (931)), that there may exist polynomial solutions to the confluent Heun equations associated with the master equation (6) which are expressed in terms of (confluent) hypergeometric functions. As it is stated in subsections IA and IC Kovacic's algorithm reduces the problem of finding Liouvillian solutions to master equation (6) to the problem of finding polynomial solutions to an associated set $\mathcal{D}$ of confluent Heun equations.

The suggestion in turn that there may exist polynomial solutions to the confluent Heun equations associated with the master equation (6) which are expressed in terms of (confluent) hypergeometric functions makes contact with the very extensive literature on the Heun equation and its various confluent forms, and in particular, with Hautot's results $\underline{60} \underline{63}$, who proved that when certain sufficient conditions are satisfied, then the confluent Heun equation admits polynomial solutions which can be expressed as a finite sum of truncated confluent hypergeometric functions of the first kind.

What makes relevant Hautot's work with our work, is as we show in subsection VIC, Hautot's sufficient conditions are satisfied in the case of the algebraically special gravitational perturbations of the Schwarzschild geometry, and in particular, are satisfied for the Liouvillian solution $\eta \int \frac{e^{-\int a}}{\eta^{2}}$ initially found by Chanadrasekhar $\underline{14}$. As we show in subsection VIC, in this case, Hautot's sufficient conditions are reduced to the condition that a determinant of fixed order is zero.

Interestingly enough, the vanishing of this determinant is equivalent to the vanishing of the Starobinsky constant, i.e., it is equivalent to the fact that the frequencies of the algebraically special gravitational perturbations of the Schwarzschild geometry take the values 
firstly found by Couch and Newman $\underline{\underline{16}}$, and then subsequently rediscovered by Wald $\underline{15}$ and by Chandrasekhar $\underline{14}$.

We note in passing that the importance of Hautot's result lies in an apparently curious fact: Confluent Heun equation, an equation with three singular points, when certain sufficient conditions are satisfied, admits polynomial solution which can be expressed as a sum of a fixed number, the number being determined by the confluent Heun equation at hand, of truncated confluent hypergeometric functions of the first kind, which satisfy the hypergeometric confluent differential equation, an equation with two singular points!

So it seems, that when Hautot's sufficient conditions are satisfied, a singularity disappears! Hautot does not give an explanation of the phenomenon. To the best of our knowledge the first systematic study and explanation of this phenomenon was given by Craster and Shanin in ${ }^{64}$. Their work is illuminating since their explanation involves isomonodromy mappings of the solution space of the confluent Heun equation. An inspiring recent study $\underline{65}$ sets the stage for a group theoretical explanation of this phenomenon with the use of tridiagonalization.

The results derived in this paper, and in particular the extension of Hautot's results we give in subsections VIIB, VIIC and VIID, provide a test bed for a verification of the results in $\underline{\underline{64}, 65}$, and even more, for extending these results and for unifying them in a coherent framework. We elaborate more on these issues in section IX.

Interestingly enough, in the problem under consideration, Hautot's sufficient conditions are nothing but the requirement that the frequencies of the gravitational perturbations of the Schwarzschild geometry are the frequencies (equation (800) of the algebraically special perturbations!

However, when we try to form Hautot's finite sum of truncated confluent of hypergeometric functions of the first kind, we immediately encounter an obstruction, since, as we show in subsection VIIB, two of the four terms of the sum are not defined in the case of the Liouvillian solution $\eta \int \frac{e^{-\int a}}{\eta^{2}}$ initially found by Chanadrasekhar $\underline{14}$.

So it appears, that oddly enough, although in the case of the the Liouvillian solution $\eta \int \frac{e^{-\int a}}{\eta^{2}}$, initially found by Chanadrasekhar $\stackrel{14}{\underline{1}}$, Hautot's sufficient conditions are satisfied, Hautot's polynomial solution to the associated confluent Heun equation does not arise in this case.

We resolve this apparent paradox in subsections VIIB, VIIC and VIID, where we introduce an extension of Hautot's results $\underline{\underline{60}} \underline{\underline{63}}$, and use this extension in order to prove that 
the confluent Heun equation, associated with Chandrasekhar's Liouvillian solution $\eta \int \frac{e^{-\int a}}{\eta^{2}}$, does admit polynomial solution which can be expressed both as a sum of truncated confluent hypergeometric functions of the first kind and as a sum of associated Laguerre polynomials, with appropriate function coefficients in each case (Theorem 4 and Theorem 5 respectively).

The Euler-Gauss hypergeometric function, the confluent hypergeometric function, and generalizations thereof, play a prominent role in many branches of physics and mathematics ${ }^{66}$. The relation of the Liouvillian solutions of the RWE, and in particular of the Chandrasekhar's algebraic special solution $\eta \int \frac{e^{-\int a}}{\eta^{2}}$, to the hypergeometric functions has not been investigated so far. It is an enigma that even publications devoted to the analytic treatment ${ }^{39}$ of the Chandrasekhar's algebraic special solution $\eta \int \frac{e^{-\int a}}{\eta^{2}}$ do not even address the problem of the relation of this solution with the hypergeometric functions.

The effective curvature potential (10) of the RWE is complicated, so the existence of Liouvillian solutions in the case of the gravitational perturbations of the Schwarzschild geometry comes as a surprise, and their expression as a product of elementary functions, one of them being a polynomial which admits an expansion both in terms of truncated confluent hypergeometric functions of the first kind (Theorem 4), and in terms of associated Laguerre polynomials (Theorem 5), even more so. This opens a new chapter in the ever active area of solvability of Schrödinger potentials ${ }^{67}-\underline{\underline{ }}$ and further study is needed in order to find out how the results we obtain in this paper are generalized to other black hole geometries, 4-dim and higher $\underline{41}$.

This paper is organised as follows. In section \some fundamental formulae are presented. In section III Kovacic's algorithm is outlined. In section IV] Kovacic's algorithm is applied to the master equation (6) which governs the evolution of the first-order perturbations of the Schwarzschild geometry. In section $\mathrm{V}$ two Liouvillian solutions of the master equation (6) are found in the gravitational case, and it is shown that both of them can be written as product of elementary functions. In section VI Hautot's results are summarized and their relation to the perturbations of the Schwarzschild geometry is given. In section VII Hautot's results are extended and the extended results are used in order to express one of the Liouvillian solutions found in section $\mathrm{V}$ as a product of elementary functions, one of them being a polynomial which can be expressed as a sum of truncated confluent hypergeometric functions of the first kind (Theorem 4), and also, it can be expressed as a sum of associated Laguerre polynomials (Theorem 5), with appropriate function coefficients in both cases. In 
section VIII we prove, save for two unresolved cases, that the Liouvillian solutions given, in the case of the gravitational perturbations, in section $\mathrm{V}$, are the only Liouvillian solutions to the master equation (6). We also compare our results with the results of C.H. ${ }^{26}$ and by using the results derived in this paper we make suggestions for a more efficient implementation of Kovacic's algorithm to any second order ODE with rational function coefficients. Finally in section [X] we state interesting open problems related to the results obtained in this paper and highlight connections of these results with inspiring new developments.

\section{FUNDAMENTAL FORMULAE}

It has been found (see, for instance, $\frac{1}{-}$ and references therein) that the equations describing scalar as well as electromagnetic or gravitational radiative perturbations on the static part of the Schwarzschild space-time can be reduced to the master equation that follows, by separating out the dependence on angles and assuming that the time dependence on the perturbations is $\exp [-\mathrm{i} \sigma t]$.

This assumption corresponds to a Fourier analysis of the perturbing field $\Phi(t, r, \theta, \phi)$ :

$$
\Phi(t, r, \theta, \phi)=\frac{1}{2 \pi} \int_{-\infty}^{\infty} \exp [-\mathrm{i} \sigma t]\left(\sum_{l} \frac{1}{r} \psi_{l}(r, \sigma) \mathrm{Y}_{l 0}(\theta, \phi)\right) \mathrm{d} \sigma .
$$

$\Phi(t, r, \theta, \phi)$ is such that from it all components of the perturbed metric tensor can be reconstructed.

Separation of the dependence on angles leads (see, for instance, $\frac{1}{-}$ ) to the equation

$$
\frac{\mathrm{d}^{2}}{\mathrm{~d} r_{*}^{2}} \psi_{l}(r)+\left[\sigma^{2}-\left(1-\frac{2 M}{r}\right)\left(\frac{l(l+1)}{r^{2}}+\frac{2 \beta M}{r^{3}}\right)\right] \psi_{l}(r)=0 .
$$

Equation (3) is the master equation which governs the evolution of first order scalar, electromagnetic and gravitational radiative perturbations on the static part of the Schwarzschild space-time. In equation (3) and throughout in this paper we use geometric unit system in which $\mathrm{c}=\mathrm{G}=1$.

$\mathrm{Y}_{l m}$ are the spherical harmonics, $r$ is the Schwarzschild radial coordinate, $r_{*}=r+$ $2 M \log \left(\frac{r}{2 M}-1\right)$ is the Regge-Wheeler "tortoise coordinate", $\sigma$ is the frequency, and $\beta=$ $-3,0,1$ and distinguishes the various types of external perturbations, gravitational, electromagnetic or massless scalar fields respectively. When a function $\mathrm{f}\left(r_{*}\right)$ is written in terms of the Schwarzschild coordinate $r$, the inverse function, $r\left(r_{*}\right)$, is to be assumed. 
The origins of these equations are reviewed by Leaver ${ }^{1}$. Let us make a few remarks relevant to our work. Regge and Wheeler ${ }^{2}$ showed how odd-parity (axial) gravitational perturbations to the geometry could be expressed in terms of the solutions of equation (3) when $\beta=-3$. A very similar equation obeyed by even-parity (polar) gravitational perturbations, but with a different potential, was derived by Zerilli13 ${ }^{13}$ Chandrasekhar ${ }^{71}, 72$ and Chandrasekhar and Detweiler ${ }^{-\underline{5}}$ subsequently showed that solutions to Zerilli's even-parity equation could be expressed in terms of the Regge-Wheeler odd-parity solutions and vice versa. In particular, the following relations hold

$$
\begin{aligned}
& {\left[\mu^{2}\left(\mu^{2}+2\right)+12 \mathrm{i} \sigma M\right] \mathrm{Z}^{(+)}=\left[\mu^{2}\left(\mu^{2}+2\right)+72 M^{2} \frac{r-2 M}{r^{2}\left(\mu^{2} r+6 M\right)}\right] \mathrm{Z}^{(-)}+12 M \frac{\mathrm{d}}{\mathrm{d} r_{*}} \mathrm{Z}^{(-)},} \\
& {\left[\mu^{2}\left(\mu^{2}+2\right)-12 \mathrm{i} \sigma M\right] \mathrm{Z}^{(-)}=\left[\mu^{2}\left(\mu^{2}+2\right)+72 M^{2} \frac{r-2 M}{r^{2}\left(\mu^{2} r+6 M\right)}\right] \mathrm{Z}^{(+)}-12 M \frac{\mathrm{d}}{\mathrm{d} r_{*}} \mathrm{Z}^{(+)},}
\end{aligned}
$$

$\left(\underline{5}\right.$, equation (61); see also ${ }^{72}$, page 162 , equations $\left.(152),(153)\right)$, where $\mu^{2}=(l-1)(l+2), \mathrm{Z}^{(+)}$ are the solutions to Zerilli's even-parity equation, and $\mathrm{Z}^{(-)} \equiv \psi_{l}(r)$ when $\beta=-3$ and $\frac{\mathrm{d}}{\mathrm{d} r_{*}}=\frac{r-2 M}{r} \frac{\mathrm{d}}{\mathrm{d} r}$.

Therefore (see equation (44)), a solution $\mathrm{Z}^{(-)}$to Regge-Wheeler's equation will yield a solution $\mathrm{Z}^{(+)}$to Zerilli's equation and vice versa (see equation (5)). Due to the particular functional dependence of $\mathrm{Z}^{(-)}$and $\mathrm{Z}^{(+)}$, if $\mathrm{Z}^{(-)}$is expressed in terms of quadratures so is $\mathrm{Z}^{(+)}$ (equation (4)) and vice versa (equation (15)). Thus it is not necessary to consider Zerilli's equation separately. The $\beta=0$ equation for electromagnetic perturbations was first derived by Wheeler ${ }^{73}$, while Ruffini, Tiomno and Vishveshwara ${ }^{74}$ showed how the two equations governing the radial parts of even-parity and odd-parity electromagnetic perturbations with a source are reduced to a single differential equation,namely, Wheeler's equation, when the source is switched off.

The lowest multipoles which can radiate are the gravitational quadrupole, the electromagnetic dipole, and the scalar monopole ${ }^{75}, 76$. The master equation (3) is not applicable for non-radiative multipoles, since the field equations are not the same in this case $\mathrm{e}^{\underline{2}} \underline{\underline{4}}, \underline{12}, \underline{73} \underline{\underline{75}}$. In the gravitational case, exact solutions both of the odd-parity and even-parity perturbation equations when $l=0,1$ can be given ${ }^{12}$. In the electromagnetic case and when $l=0$, the smooth allowed transition from a Schwarzschild to a Reisner-Nordstrom geometry, which takes place through the capture of a charge particle in a given Schwarzschild background, has been studied by Hanni and Ruffini $\underset{77}{ }$. Thus, in our investigation, the angular harmonic 
index $l$ will take the values $2,3, \ldots$ in the gravitational case, $1,2, \ldots$ in the electromagnetic case, and $0,1,2, \ldots$ in the scalar case.

For the purposes of our study we transform the master equation (3) into the standard Schrödinger form, with the Schwarzschild coordinate $\mathrm{r}$ as the independent variable. To accomplish this, first we change the independent variable from $r_{*}$ to $r$, and then we eliminate the term containing the first derivative by a standard transformation of the dependent variable. The details are as follows.

By changing the independent variable from $r_{*}$ to $r$, the master equation (3) is rewritten as follows

$$
\frac{\mathrm{d}^{2}}{\mathrm{~d} r^{2}} \psi_{l}(r)+\frac{2 M}{r(r-2 M)} \frac{\mathrm{d}}{\mathrm{d} r} \psi_{l}(r)-\left[\frac{s^{2} r^{4}}{4 r^{2}(r-2 M)^{2}}+\frac{l(l+1)}{r(r-2 M)}+\frac{2 \beta M}{r^{2}(r-2 M)}\right] \psi_{l}(r)=0,
$$

where

$$
s^{2}=-4 \sigma^{2}
$$

will prove a convenient substitution later on. When $\beta=-3$, equation (66), or equivalently equation (3) , is known as the Regge-Wheeler equation² (equation (6) is identical to equation (23), page $\left.144^{72}\right)$ ). Equation (66) does not change if $s$ is replaced with $-s$. Thus, in case of real $s$, only non-negative values of $s$ need to be considered.

The term containing the first derivative of the dependent variable in (6) can be eliminated by a standard transformation of the dependent variable. To obtain the Schrödinger - like diffential equation in $\mathrm{r}$, we perform this transformation in equation (6)

$$
\psi_{l}^{\prime}(r)=\left(1-\frac{2 M}{r}\right)^{\frac{1}{2}} \psi_{l}(r)
$$

The result is

$$
\frac{\mathrm{d}^{2}}{\mathrm{~d} r^{2}} \psi_{l}^{\prime}+\mathrm{R}_{l}(r) \psi_{l}^{\prime}=0
$$

where

$$
\mathrm{R}_{l}(r)=\frac{r^{2}}{(r-2 M)^{2}}\left[-\frac{s^{2}}{4}-\mathrm{V}_{l}(r)+\frac{2 M}{r^{3}}-\frac{3 M^{2}}{r^{4}}\right]
$$

and

$$
\mathrm{V}_{l}(r)=\left(1-\frac{2 M}{r}\right)\left[\frac{l(l+1)}{r^{2}}+\frac{2 \beta M}{r^{3}}\right] .
$$

By using Kovacic's algorithm on the equation (9), we will investigate all its solutions in terms of quadratures, and hence, due to (8) , all the Liouvillian solutions of the master equation (6). 
For convenience, we rewrite equation (9) in the form

$$
\mathcal{O}_{(r, s, M)} \psi_{l}^{\prime}=0
$$

where $\mathcal{O}_{(r, s, M)}$ is the linear operator

$$
\mathcal{O}_{(r, s, M)}=\frac{\mathrm{d}^{2}}{\mathrm{~d} r^{2}}+\mathrm{R}_{l}(r)
$$

The subscript $(r, s, M)$ in $\mathcal{O}_{(r, s, M)}$ emphasizes the dependence of $\mathcal{O}_{(r, s, M)}$ on the parameters $r, s$ and $M$.

Regarding the parameter $M$, the mass of the black hole, which appears in equation (9) we note the following: The transformation

$$
r=\rho k M, \quad s=\frac{\tau}{k M}
$$

where $k$ is a positive integer, leaves equation (9) quasi-invariant, it replaces the parameter $M$ with $\frac{1}{k}$. Thus we have

$$
\mathcal{O}_{(r, s, M)} \psi_{l}^{\prime}=0 \Leftrightarrow \mathcal{O}_{\left(\rho, \tau, \frac{1}{k}\right)} \psi_{l}^{\prime}=0
$$

We conclude that by making use of the transformation (14) we can set $M$, without loss of generality, to be any positive number. For simplicity, henceforth, we choose $k$ in (14) to be equal to one, i.e., for simplicity, throughout in this paper, we set $M=1$. The same remark, regarding the parameter $M$, applies also to equations (3) and (6). The transformation (14) and the subsequent equivalence (15) are of crucial importance in subsections VIIIF and VIIIG. In subsection VIIIF we compare our results with the results of C.H. $\stackrel{26}{ }$ on the solution of problem 3, mentioned in subsection IA. In subsection VIIIG we make some comments on the implementation of Kovacic's algorithm.

In section III we give Kovacic's algorithm in some detail and in section IV we give in detail the application of Kovacic's algorithm. This is useful and necessary for a number of reasons:

1. It facilitates the clear and detailed exposition of the results presented in this paper.

2. It allows and makes easier the comparison of our results with the results of other researchers on the problem of finding all Liouvillian solutions to the perturbation equations of the Schwarzschild geometry which we examine in this paper. 
3. It facilitates the comparison of our results with other results on the problem of finding all Liouvillian solutions to the perturbation equations of other black hole geometries 4-dim and higher $\stackrel{41}{ }$.

Regarding the second reason we note that C.H..$^{26}$ present the version of Kovacic's algorithm they use, its application, and their results on the problem of finding all Liouvillian solutions to the perturbation equations of the Schwarzschild geometry in a succinct form. This makes difficult to trace the origin of some differences in our and their results on the solution of problem 3, mentioned in subsection IA. These differences are stated in subsection VIIIF. We have checked our calculations several times and we have found them correct. On the other hand the succinct form of presentation of the results in $^{26}$ has prohibited us from repeating all their calculations and so the origin of the differences remains obscure.

\section{KOVACIC'S ALGORITHM}

Kovacic's algorithm 18 finds a "closed-form" solution of the differential equation (11)

$$
y^{\prime \prime}+a y^{\prime}+b y=0
$$

where $a$ and $b$ are rational functions of a complex variable $x$, provided a "closed-form" solution exists. The algorithm is so arranged that if no solution is found, then no solution can exist. The "closed-form" solution means a Liouvillian solution, i.e. one that can be expressed in terms of algebraic functions, exponentials and indefinite integrals. (As functions of a complex variable are considered, trigonometric functions need not be mentioned explicitly, as they can be written in terms of exponentials. Logarithms are indefinite integrals and hence are allowed). A more precise definition involves the notion of Liouvillian field 18 .

Let $\eta$ be a (non-zero) Liouvillian solution of the differential equation (1). It follows that every solution of this differential equation is Liouvillian. Indeed the method of reduction of order produces a second solution, namely

$$
\eta \int \frac{e^{-\int a}}{\eta^{2}}
$$

This second solution is evidently Liouvillian and the two solutions are linearly independent. Thus any solution, being a linear combination of these two, is Liouvillian. 
A well-known change of dependent variable may be used to eliminate the term involving $y^{\prime}$ from the differential equation (1). Let

$$
z=e^{\frac{1}{2} \int a} y
$$

(this is nothing else but the transformation (8) $)$. Then

$$
z^{\prime \prime}+\left(b-\frac{1}{4} a^{2}-\frac{1}{2} a^{\prime}\right) z=0
$$

(thus $\mathrm{R}_{l}(r)$ in equation (10) equals $b-\frac{1}{4} a^{2}-\frac{1}{2} a^{\prime}$ where $a=\frac{2}{r(r-2)}$ and $\quad b=$ $\left.-\left[\frac{s^{2} r^{4}}{4 r^{2}(r-2)^{2}}+\frac{l(l+1)}{r(r-2)}+\frac{2 \beta}{r^{2}(r-2)}\right]\right)$. Equation (18) still has rational function coefficients and evidently (see equation (17) $) y$ is Liouvillian if and only if $z$ is Liouvillian. Thus no generality is lost by assuming that the term involving $y^{\prime}$ is missing from the differential equation (1).

Before giving the main result obtained by Kovacic ${ }^{18}$ we first introduce some notation and some terminology. $C$ denotes the complex numbers and $C(x)$ the rational functions over $C$. An algebraic function $\omega$ of $x$ is of degree $\mathrm{k}$, where $\mathrm{k}$ is a positive integer, when $\omega$ solves an irreducible algebraic equation

$$
\Pi(\omega, x)=\sum_{\mathrm{i}=0}^{\mathrm{k}} \frac{\mathrm{P}_{\mathrm{i}}(x)}{(\mathrm{k}-\mathrm{i}) !} \omega^{\mathrm{i}}=0
$$

where $\mathrm{P}_{\mathrm{i}}(x)$ are rational functions of $x$. Let $\nu \in C(x)$ (to avoid triviality, $\nu \notin C$ ).

Then the following holds $\frac{18}{}$

\section{Theorem 1 Equation}

$$
y^{\prime \prime}=\nu y, \quad, \quad \nu \in C(x)
$$

has a Liouvillian solution if and only if it has a solution of the form

$$
y=e^{\int \omega \mathrm{d} x}
$$

where $\omega$ is an algebraic function of $x$ of degree 1,2,4,6 or 12.

The search of Kovacic's algorithm for $\Pi(\omega, x)$ is based on the knowledge of the poles of $\nu$ and consists in constructing and testing a finite number of possible candidates for $\Pi(\omega, x)$. If no $\Pi(\omega, x)$ is found then the differential equation (20) has no Liouvillian solutions. If such a $\Pi(\omega, x)$ is found and $\omega$ is a solution of the equation (19) then the function $\eta=e^{\int \omega \mathrm{d} x}$ is a Liouvillian solution of (201). 
If

$$
\nu(x)=\frac{s(x)}{t(x)}
$$

with $s, t \in C[x],(C[x]$ denotes the polynomials over $\mathrm{C})$, relatively prime, then the poles of $\nu$ are the zeros of $t(x)$ and the order of the pole is the multiplicity of the zero of $t$. The order of $\nu$ at $\infty, \mathrm{o}(\infty)$, is defined as

$$
\mathrm{o}(\infty)=\max \left(0,4+\mathrm{d}^{\mathrm{o}} s-\mathrm{d}^{\mathrm{o}} t\right)
$$

where $\mathrm{d}^{\mathrm{o}} s$ and $\mathrm{d}^{\mathrm{o}} t$ denote the leading degree of $s$ and $t$ respectively (for a justification of this definition see ${ }^{19}$ page 10; Kovacic originally gave a different definition, see ${ }^{18}$ page 8$)$. We

give now an outline of Kovacic's algorithm. This is the outline of an improved version of the algorithm given by Duval and Loday-Richaud ${ }^{19}$.

\section{A. The algorithm}

Notations. Let $\mathrm{L}_{\max }=\{1,2,4,6,12\}$ and let $h$ be the function defined on $\mathrm{L}_{\max }$ by

$$
h(1)=1, h(2)=4, \quad h(4)=h(6)=h(12)=12 .
$$

Input: A rational function

$$
\nu(x)=\frac{s(x)}{t(x)} \quad(\text { equation }(22)) .
$$

The polynomials $s, t \in C[x]$ are supposed to be relatively prime.

The differential equation under consideration is

$$
y^{\prime \prime}-\nu y=0 \quad(\text { equation }(\underline{20}))
$$

First step: The set $L$ of possible degrees of $\omega$.

We are interested in equation (20) where $\nu(x)$ is given by (22).

1a. If $t(x)=1$, set $m=0$. Otherwise, factorize $t(x)$

$$
t=t_{1} t_{2}^{2} t_{3}^{3} \ldots t_{m}^{m}
$$

where the $t_{\mathrm{i}}, \mathrm{i}=1,2, \ldots, m$, are relatively prime two by two and each $t_{\mathrm{i}}$ either is equal to one or has simple zeros. Let

$$
\Gamma^{\prime}=\{c \in C, t(c)=0\} \text { and } \Gamma=\Gamma^{\prime} \cup\{\infty\}
$$


where $\cup$ denotes set-theoretic union. Associate orders with the elements of $\Gamma$ :

$$
\mathrm{o}(c)=\mathrm{i}
$$

for all $c \in \Gamma^{\prime}$,where $\mathrm{i}$ is such that $t_{\mathrm{i}}(c)=0$, and

$$
\mathrm{o}(\infty)=\max \left(0,4+\mathrm{d}^{\mathrm{o}} s-\mathrm{d}^{\mathrm{o}} t\right) .
$$

Let

$$
m^{+}=\max (m, o(\infty))
$$

For $0 \leq \mathrm{i} \leq m^{+}$let

$$
\Gamma_{\mathrm{i}}=\{c \in \Gamma \mid \mathrm{o}(c)=\mathrm{i}\} .
$$

1b. If $m^{+} \geq 2$ define $\gamma_{2}$ and $\gamma$ by

$$
\gamma_{2}=\left|\Gamma_{2}\right| \text { and } \gamma=\gamma_{2}+\left|\cup \Gamma_{\mathrm{k}}\right|, \mathrm{k} \text { is odd, and } 3 \leq \mathrm{k} \leq \mathrm{m}^{+}
$$

respectively, where, if $\mathrm{S}$ is a set then $|\mathrm{S}|$ denotes the number of elements of $\mathrm{S}$.

1c. For each $c \in \Gamma_{2}$, if $c \neq \infty$, calculate the number $\mathrm{a}_{c}$ so that

$$
\nu(x)=\frac{\mathrm{a}_{c}}{(x-c)^{2}}+\mathrm{O}\left(\frac{1}{x-c}\right)
$$

where the O symbol has its usual meaning, $f(x)=\mathrm{O}(g(x))$ if $|f(x)| \leq \mathrm{M} g(x)$ for some positive constant M; $g(x)$ is assumed to be positive. The coefficient $\mathrm{a}_{c}$ in (31) is the coefficient of $1 /(x-c)^{2}$ in the Laurent series expansion of $\nu(x)$ about $c$.

1d. Construct $\mathrm{L}$, a subset of $\mathrm{L}_{\max }$, as follows

$$
\begin{gathered}
1 \in \mathrm{L} \Longleftrightarrow \gamma=\gamma_{2}, \\
2 \in \mathrm{L} \Longleftrightarrow \gamma \geq 2, \text { and, } \\
4,6 \text { and } 12 \in \mathrm{L} \Longleftrightarrow m^{+} \leq 2 .
\end{gathered}
$$

1e. If $\mathrm{L}=\emptyset$ go to the stage of the algorithm END. Otherwise, let $\mathrm{n}$ be equal to the smallest element of L.

Second step: The sets $\mathrm{E}_{c}$ associated to the singular points.

Construction of the sets $\mathrm{E}_{c}, c \in \Gamma$.

2a. When $\mathrm{n}=1$, for each $c \in \Gamma_{2}$, define $\mathrm{E}_{c}$ as follows

$$
\mathrm{E}_{c}=\left\{\frac{1}{2}\left(1 \pm \sqrt{1+4 \mathrm{a}_{c}}\right)\right\} \text {. }
$$


2b. When $\mathrm{n} \geq 2$, for each $c \in \Gamma_{2}$, define $\mathrm{E}_{c}$ as follows

$$
\mathrm{E}_{\mathrm{c}}=\left\{\frac{h(\mathrm{n})}{2}\left(1-\sqrt{1+4 \mathrm{a}_{c}}\right)+\frac{h(\mathrm{n})}{\mathrm{n}} \mathrm{k} \sqrt{1+4 \mathrm{a}_{c}} \mid \mathrm{k}=0,1, \ldots, \mathrm{n}\right\} \cap Z,
$$

where $\cap$ denotes set-theoretic intersection and $Z$ denotes the set of integers.

2c. When $\mathrm{n}=1$, for each $c \in \Gamma_{2 \mathrm{q}}$ with $\mathrm{q} \geq 2$, calculate one of the two "square roots" $[\sqrt{\nu}]_{c}$ of $\nu$ defined as follows:

If $c=\infty$,

$$
[\sqrt{\nu}]_{\infty}=\mathrm{a}_{\infty} x^{\mathrm{q}-2}+\sum_{\mathrm{i}=0}^{\mathrm{q}-3} \delta_{\mathrm{i}, \infty} x^{\mathrm{i}}
$$

and

$$
\nu-[\sqrt{\nu}]_{\infty}^{2}=-\beta_{\infty} x^{\mathrm{q}-3}+\mathrm{O}\left(x^{\mathrm{q}-4}\right)
$$

Let

$$
\mathrm{E}_{\infty}=\left\{\frac{1}{2}\left(\mathrm{q}+\epsilon \frac{\beta_{\infty}}{\mathrm{a}_{\infty}}\right) \mid \epsilon= \pm 1\right\} .
$$

Define a function "sign" $\mathrm{S}$ with domain $\mathrm{E}_{\infty}$ as follows

$$
\mathrm{S}\left(\frac{1}{2}\left(\mathrm{q}+\epsilon \frac{\beta_{\infty}}{\mathrm{a}_{\infty}}\right)\right)= \begin{cases}\epsilon & \text { if } \beta_{\infty} \neq 0 \\ 1 & \text { otherwise }\end{cases}
$$

2d. When $\mathrm{n}=2$, for each $c \in \Gamma_{\mathrm{q}}$ with $\mathrm{q} \geq 3$, define $\mathrm{E}_{c}$ as follows

$$
\mathrm{E}_{c}=\{\mathrm{q}\}
$$

In equation (37) $[\sqrt{\nu}]_{\infty}$ is the sum of terms involving $x^{\mathrm{i}}$ for $0 \leq \mathrm{i} \leq \mathrm{q}-2$ in the Laurent series for $\sqrt{\nu}$ at $\infty$. In practice, one would not form the Laurent series for $\sqrt{\nu}$, but rather would determine $[\sqrt{\nu}]_{\infty}$ by using undetermined coefficients, i.e. by equating $\left([\sqrt{\nu}]_{\infty}\right)^{2}=\left(\mathrm{a}_{\infty} x^{\mathrm{q}-2}+\delta_{\nu-3, \infty} x^{\mathrm{q}-3}+\ldots+\delta_{0, \infty}\right)^{2}$ with the corresponding part of the Laurent series expansion of $\nu$ at $\infty$. There are two possibilities for $[\sqrt{\nu}]_{\infty}$, one being the negative of the other, and any of these may be chosen. In equation (38), $\nu$ denotes its Laurent series expansion at $\infty$. This equation defines $\beta_{\infty}$.

Third step: Possible degrees for $P$ and possible values for $\theta$.

3a. For each family $\underline{\mathrm{e}}=\left(\mathrm{e}_{c}\right)_{c \in \Gamma}$ of elements $\mathrm{e}_{c} \in \mathrm{E}_{c}$ calculate

$$
\mathrm{d}(\underline{\mathrm{e}})=\mathrm{n}-\frac{\mathrm{n}}{h(\mathrm{n})} \sum_{c \in \Gamma} \mathrm{e}_{c}
$$


3b. Retain the families $\underline{e}$ which are such that

i) $\mathrm{d}(\underline{\mathrm{e}}) \in N$ where $N$ is the set of non - negative integers and

ii) when $\mathrm{n}=2$ or 4 at least two $\mathrm{e}_{\mathrm{c}}$ are odd numbers in each family.

If none of the families $\underline{e}$ is retained, go to the stage of the algorithm $\underline{\text { CONTINUATION. }}$

3c. For each family e retained from step $\mathbf{3 b}$, form the rational function

$$
\begin{aligned}
\theta=\frac{\mathrm{n}}{h(\mathrm{n})} \sum_{c \in \Gamma^{\prime}} \frac{\mathrm{e}_{c}}{x-c}+ & \delta_{\mathrm{n}}^{1} \sum_{c} \mathrm{~S}\left(\mathrm{e}_{c}\right)[\sqrt{r}]_{c}, \\
\mathrm{q} & \geq 2
\end{aligned}
$$

where $\delta_{\mathrm{n}}^{1}$ is the Kronecker symbol.

Fourth Step: Tentative computation of $P$.

Search for a polynomial P of degree d (as defined in step 3a) such that

$$
\begin{aligned}
& \mathrm{P}_{\mathrm{n}}=-\mathrm{P} \\
& \cdots \\
& \mathrm{P}_{\mathrm{i}-1}=-\mathrm{P}_{\mathrm{i}}^{\prime}-\theta \mathrm{P}_{\mathrm{i}}-(\mathrm{n}-\mathrm{i})(\mathrm{i}+1) \nu \mathrm{P}_{\mathrm{i}+1} \\
& \cdots \\
& \mathrm{P}_{-1}=0
\end{aligned}
$$

where the prime in $\mathrm{P}^{\prime}(x)$ denotes differentiation with respect to the independent variable $x$.

\section{Output:}

OUTPUT1: If such a polynomial is found and $\omega$ is a solution of the irreducible algebraic equation

$$
\left.\sum_{\mathrm{i}=0}^{\mathrm{n}} \frac{\mathrm{P}_{\mathrm{i}}(x)}{(\mathrm{n}-\mathrm{i}) !} \omega^{\mathrm{i}}=0 \quad \text { (equation (19) }\right) \text {, }
$$

where the rational functions $\mathrm{P}_{\mathrm{i}}(x)$ are defined in (44), then the function $\eta=e^{\int \omega}$ is a Liouvillian solution of the equation under consideration

$$
y^{\prime \prime}=\nu y \quad(\text { equation }(20)) \text {. }
$$

If no such polynomial is found for any family retained from step $\mathbf{3 b}$, go to the stage of algorithm CONTINUATION.

CONTINUATION : If $\mathrm{n}$ is different from the largest element of $\mathrm{L}$ then set $\mathrm{n}$ equal to the next (in increasing order) element of L and go to Step 2. 
OUTPUT2: The equation $y^{\prime \prime}=\nu y$ has no Liouvillian solutions.

We sketched Kovacic's algorithm in the form needed in the subsequent section. Now, besides the original formulation of this algorithm $\frac{18}{2}$ we have its several versions, improvements ${ }^{19-21}$, and extensions to higher order equations $22-24$. For example, the formulation of Kovacic's algorithm given in ${ }^{21}$ is different both from its original formulation ${ }^{18}$ and from the formulation of Kovacic's algorithm given in this paper. It seems that the formulation in 21 is much more convenient for computer implementation and in fact has been implemented in Maple.

However, it seems that for differential equations with simple structure of singularities, and depending on parameters, the formulation of the algorithm given in this paper, which is an outline of the formulation given in $\frac{19}{}$, is well suited. Moreover, the original formulation of the algorithm $\frac{18}{}$ consists in fact of three separated algorithms each of them repeating similar

steps. In $\frac{19}{}$ one can find a modification of the original formulation unifying and improving these three algorithms in one. This form is very convenient for applications and it is the one employed here.

\section{APPLICATION OF KOVACIC'S ALGORITHM}

Input: Equation (9) is the same as equation (20) with

$$
\begin{aligned}
\nu & =-\mathrm{R}_{l}(r)=\frac{r^{2}}{(r-2)^{2}}\left[\frac{s^{2}}{4}+\left(1-\frac{2}{r}\right)\left(\frac{l(l+1)}{r^{2}}+\frac{2 \beta}{r^{3}}\right)-\frac{2}{r^{3}}+\frac{3}{r^{4}}\right] \\
& =\frac{\frac{s^{2}}{4} r^{4}+l(l+1) r^{2}+2[\beta-l(l+1)-1] r+3-4 \beta}{r^{2}(r-2)^{2}} \equiv \frac{s(r)}{t(r)} .
\end{aligned}
$$

The partial fraction expansion for $\nu$ is the following

$$
\begin{aligned}
\nu(r)= & \frac{s^{2}}{4}+\frac{1}{4}(3-4 \beta) \frac{1}{r^{2}}+\frac{\frac{1}{4}(-2 \beta-2 l(l+1)+1)}{r} \\
& +\frac{\frac{1}{4}\left(4 s^{2}-1\right)}{(r-2)^{2}}+\frac{\frac{1}{4}\left(4 s^{2}+2 l(l+1)+2 \beta-1\right)}{r-2} .
\end{aligned}
$$

We now apply Kovacic's algorithm to equation (9):

\section{First step:}


1a. From (45) we obtain

$$
\begin{array}{rcc}
t(r)=r^{2}(r-2)^{2} . \text { Hence } m=2 \text { and } & \Gamma^{\prime}=\{0,2\}, & \Gamma=\{0,2, \infty\} \\
\mathrm{o}(0)= & \mathrm{o}(2)= & 2 \\
\mathrm{~d}^{\mathrm{o}} s=\mathrm{d}^{\mathrm{o}} t=4, & \mathrm{o}(\infty)=4 \text { and } \quad & m^{+}=4 \\
\Gamma_{\mathrm{o}}=\Gamma_{1}=\emptyset, \Gamma_{2}=\{0,2\}, \Gamma_{3}=\emptyset \text { and } \Gamma_{4}=\{\infty\} .
\end{array}
$$

1b. Equations (47) give

$$
\gamma_{2}=2 \quad \text { and } \quad \gamma=\gamma_{2}=2 .
$$

1c. From (46) we obtain

$$
\mathrm{a}_{\mathrm{o}}=\frac{1}{4}(3-4 \beta) \quad \text { and } \quad \mathrm{a}_{2}=\frac{1}{4}\left(4 s^{2}-1\right) .
$$

1d. Equations (48) imply

$$
\mathrm{L}=\{1,2\}
$$

1 e.

$$
\mathrm{n}=1 .
$$

\section{Second step:}

2a. We are considering the case $\mathrm{n}=1$. The roots $0,2 \in \Gamma_{2}$ and therefore by using equations (35), (49) one finds that

$$
\mathrm{E}_{0}=\left\{\frac{1}{2}\left(1 \pm \sqrt{1+4 \mathrm{a}_{\mathrm{o}}}\right)\right\}=\left\{\frac{1}{2} \pm \sqrt{1-\beta}\right\} .
$$

Equation (52) implies

$$
\begin{gathered}
\mathrm{E}_{0}=\left\{\frac{5}{2},-\frac{3}{2}\right\} \text { when } \beta=-3, \\
\mathrm{E}_{0}=\left\{\frac{3}{2},-\frac{1}{2}\right\} \text { when } \beta=0, \\
\mathrm{E}_{0}=\left\{\frac{1}{2}\right\} \text { when } \beta=1 .
\end{gathered}
$$

Combining equations (35) and (49) yields

$$
\mathrm{E}_{2}=\left\{\frac{1}{2}\left(1 \pm \sqrt{1+4 \mathrm{a}_{2}}\right)\right\}=\left\{\frac{1}{2}+s, \frac{1}{2}-s\right\} .
$$


2b. It is not applicable since $n=1$.

2c. $\infty \in \Gamma_{4}$ and therefore

$$
q=2 \text {. }
$$

Equations (37) and (57) give

$$
[\sqrt{\nu}]_{\infty}=\mathrm{a}_{\infty}
$$

From equations (45) and (58) we obtain

$$
\left([\sqrt{\nu}]_{\infty}\right)^{2}=\mathrm{a}_{\infty}^{2}=\frac{s^{2}}{4}
$$

We choose

$$
\mathrm{a}_{\infty}=\frac{s}{2}
$$

Combining equations (38), (45) and (59) yields

$$
\beta_{\infty}=-s^{2}
$$

Making use of equations (39), (157), (60), and (61), one readily finds that

$$
\mathrm{E}_{\infty}=\{1-s, 1+s\}
$$

Combining equations (40), (57), (60), and (62), yields

$$
\mathrm{S}(1-s)=1 \text { and } \mathrm{S}(1+s)=-1
$$

2d. It is not applicable since $\mathrm{n}=1$.

\section{Third Step:}

3a. For each family $\underline{\mathrm{e}}=\left(\mathrm{e}_{c}\right)_{c \in \Gamma}$ of elements $\mathrm{e}_{c} \in \mathrm{E}_{c}$ we calculate the degree $\mathrm{d}(\underline{\mathrm{e}})$ of the corresponding, prospective polynomial P. The sets $\mathrm{E}_{c}$ are given by equations (52), (56) and (62). Since $\mathrm{E}_{\mathrm{o}}$ depends on the value of $\beta$ we distinguish three cases:

$$
\beta=-3 \quad \text { (Gravitational case) }
$$




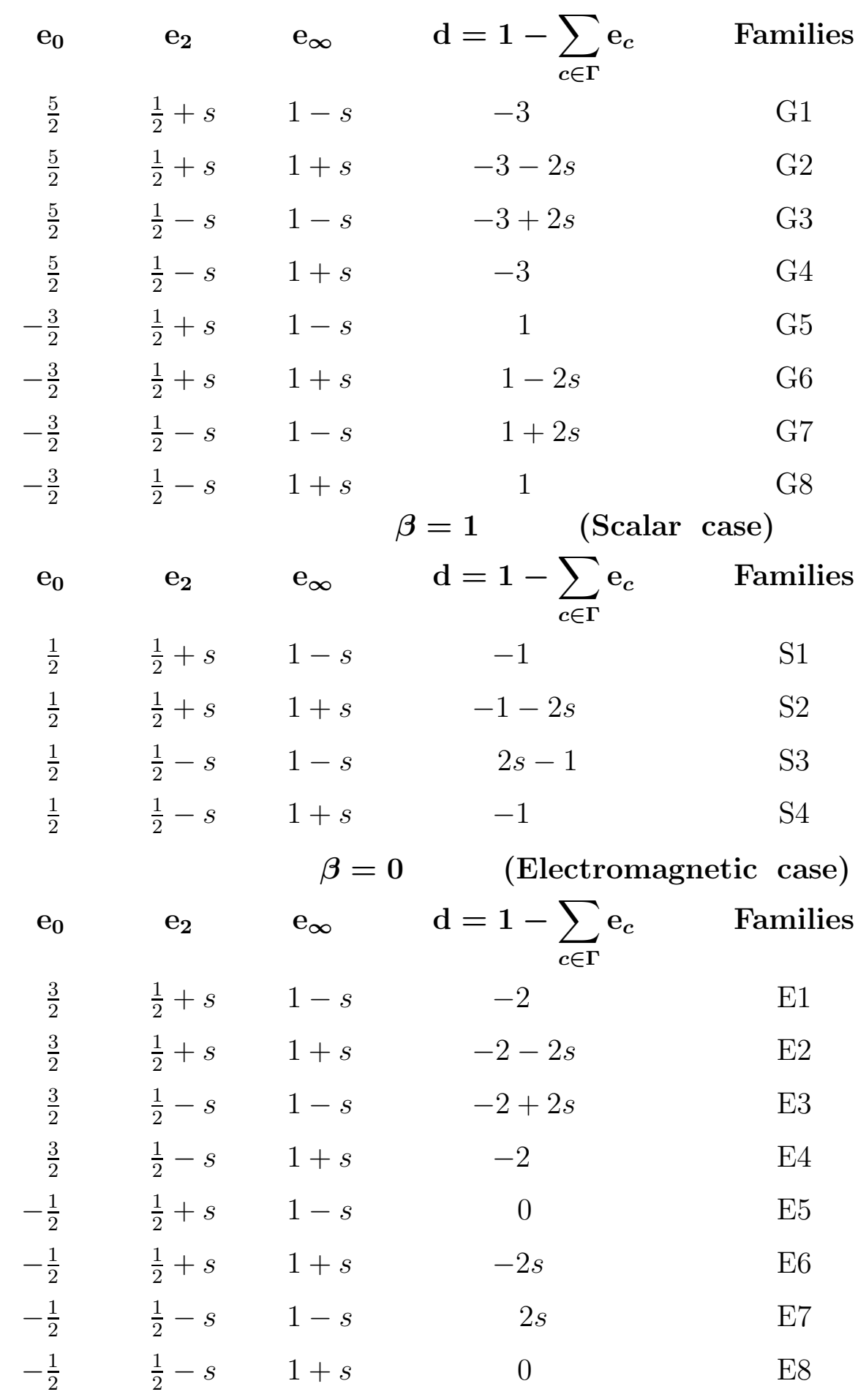

In the last column we enumerated the different families arising within each of the three cases.

3b. i) If $\mathrm{d}$ is a non-negative integer, the family should be retained, otherwise the family is discarded. Thus the families G1, G4, E1, E4, S1 and S4 are discarded. It is assumed that $s$ is non-negative and therefore the families G2, E2 and S2 are also discarded since for all of them $\mathrm{d}$ becomes negative for the non-negative values of $s$. For the family 
G6 there are only two non-negative values of $s$ for which d becomes non-negative, $\mathrm{d}=0$ when $s=1 / 2$ and $\mathrm{d}=1$ when $s=0$, and, for the family E6 there is only one, $\mathrm{d}=0$ when $s=0$. We easily find that for these values of $s$ the families G6 and E6 do not give rise to any Liouvillian solutions. A quick check shows that the same result holds for the families G5, E5 and E8. Therefore the families which remain for consideration are the following: G3, G7, G8, E3, E7 and S3.

ii) It is not applicable since $n=1$.

3c. For each family retained from step $\mathbf{3 b}$, we form the rational function $\theta$ given by equation (43). Since $n=1, \Gamma_{2}=\{0,2\}$ and $\Gamma_{4}=\{\infty\}$ equation (43) implies

$$
\theta=\frac{\mathrm{e}_{0}}{r}+\frac{\mathrm{e}_{2}}{r-2}+\mathrm{S}\left(\mathrm{e}_{\infty}\right)[\sqrt{\nu}]_{\infty}
$$

where $\mathrm{e}_{c}$ denotes any element of $\mathrm{E}_{c}, \mathrm{~S}\left(\mathrm{e}_{\infty}\right)$ are given by equations (63) and $[\sqrt{\nu}]_{\infty}$ is given by equation (60). By making use of equation (64) for each of the retained families we obtain

$$
\begin{aligned}
& \beta=-3 \quad \text { (Gravitational case) } \\
& \theta \\
& \frac{5 / 2}{r}+\frac{\frac{1}{2}-s}{r-2}+\frac{s}{2} \\
& \frac{-3 / 2}{r}+\frac{\frac{1}{2}-s}{r-2}+\frac{s}{2} \\
& \frac{-3 / 2}{r}+\frac{\frac{1}{2}-s}{r-2}-\frac{s}{2} \\
& \beta=1 \quad \text { (Scalar case) } \\
& \frac{1 / 2}{r}+\frac{\frac{1}{2}-s}{r-2}+\frac{s}{2} \\
& \beta=0 \quad \text { (Electromagnetic case) } \\
& \frac{3 / 2}{r}+\frac{\frac{1}{2}-s}{r-2}+\frac{s}{2} \\
& \frac{-1 / 2}{r}+\frac{\frac{1}{2}-s}{r-2}+\frac{s}{2}
\end{aligned}
$$

\section{Families}

\section{Families}

\section{Fourth step - Output:}


For $n=1$ equations (44) imply

$$
\begin{gathered}
\mathrm{P}_{1}=-\mathrm{P}, \\
\mathrm{P}_{0}=\mathrm{P}^{\prime}+\theta \mathrm{P},
\end{gathered}
$$

and

$$
\mathrm{P}_{-1}=0=\mathrm{P}^{\prime \prime}+2 \theta \mathrm{P}^{\prime}+\left(\theta^{2}+\theta^{\prime}-\nu\right) \mathrm{P} .
$$

Combining equations (19), (71) and (72) yields

$$
\omega=\frac{\mathrm{P}^{\prime}}{\mathrm{P}}+\theta
$$

For each of the retained families we search for a polynomial $\mathrm{P}$ of degree $\mathrm{d}$ (as defined in step 3a) such that equation (73) is satisfied. If such a polynomial $\mathrm{P}$ is found then $\omega$ is given by equation (74) and the function

$$
\eta=y=e^{\int \omega \mathrm{d} r}=e^{\int\left(\frac{\mathrm{P}^{\prime}}{\mathrm{P}}+\theta\right) \mathrm{d} r}=\mathrm{P} e^{\int \theta \mathrm{d} r}
$$

is a Liouvillian solution of equation (9).

In section $\mathrm{V}$ it will be shown that the families G7 and G8 give the solutions found by Chandrasekhar $\underline{14}$. Finally, the families G3, E3, E7 and S3 are considered in section VIII. Now the algorithm can be considered complete when $\mathrm{n}=1$.

CONTINUATION: The analysis of the case $n=2$ is given in Appendix $\mathrm{A}$.

\section{THE FAMILIES G7 AND G8}

In this section we prove that the family G8 gives the Liouvillian solution $\chi$ and more

importantly we also prove that the family G7 gives the Liouvillian solution $\chi \int \frac{\mathrm{d} r_{*}}{\chi^{2}}$. As a spin off, in subsection $\mathrm{VD}$, we prove that the integral $\int \frac{\mathrm{d} r_{*}}{\chi^{2}}$ has an elementary functions answer for every value of the angular harmonic index $l, l=2,3, \ldots$. Consequently, given the functional form of $\chi$ (equations (76) and (79) ), the Liouvillian solution $\chi \int \frac{\mathrm{d} r_{*}}{\chi^{2}}$ has an elementary functions answer for every value of the angular harmonic index $l, l=2,3, \ldots$. The Liouvillian solutions $\chi$ and $\chi \int \frac{\mathrm{d} r_{*}}{\chi^{2}}$ were firstly found by Chandrasekhar 14 in the case of the first order gravitational perturbations of the Schwarzschild geometry. We start by describing Chandrasekhar's Liouvillian solutions. 


\section{A. Chandrasekhar's Liouvillian solutions}

Chandrasekhar found 14 "closed-form" solutions to the Regge-Wheeler and Zerilli equations. These solutions correspond to algebraically special perturbations, i.e. perturbations in which we have only incoming $\left(\Psi_{0} \neq 0\right)$ or only outgoing $\left(\Psi_{4} \neq 0\right)$ waves. The vanishing of the Starobinsky constant, which is the necessary and sufficient condition for the existence of these solutions, implies that the frequency $\sigma$ of the perturbations takes pure imaginary values.

The algebraically special solutions ${ }^{43}$, initially found by Chandrasekhar, to the RWE, which describe outgoing waves, are the following

$$
\chi=\frac{\mu^{2} r+6}{r} \mathrm{e}^{-\mathrm{i} \sigma r_{*}}
$$

and

$$
\chi \int \frac{\mathrm{d} r_{*}}{\chi^{2}}
$$

where,

$$
\begin{gathered}
\mu^{2}=(l-1)(l+2) \quad l=2,3, \ldots, \\
r_{*}=r+2 \log \left(\frac{r}{2}-1\right) \text { or equivalently } \frac{\mathrm{d}}{\mathrm{d} r_{*}}=\left(1-\frac{2}{r}\right) \frac{\mathrm{d}}{\mathrm{d} r},
\end{gathered}
$$

and,

$$
\sigma_{0} \equiv \mathrm{i} \sigma=\frac{\mu^{2}\left(\mu^{2}+2\right)}{12}=\frac{l(l-1)(l+1)(l+2)}{12} .
$$

Once the solution (76) is derived the method of reduction of order produces the solution (77) (cf. equation (16) with $a=0$ ). Now we prove the following:

\section{B. The family G8 gives the Liouvillian solution $\chi$}

Proposition 1 The family G8 gives rise to the solution $\chi=\frac{\mu^{2} r+6}{r} \mathrm{e}^{-\mathrm{i} \sigma r_{*}}$.

Proof We need to find a polynomial $\mathrm{P}=r+\mathrm{k}$ such that equation (73) is satisfied, where, $\theta$ now is given by equation (67). Combination of equations (45), (67) and (173) yields

$$
(-\mathrm{k} s+l(l+1)) r+(l+2)(l-1) \mathrm{k}-6=0 .
$$

From equation (81) it follows that

$$
\mathrm{k}=\frac{6}{(l+2)(l-1)} \quad \text { and } \quad s=\frac{l(l-1)(l+1)(l+2)}{6} .
$$


Since a polynomial solution $\mathrm{P}=r+\mathrm{k}$ to equation (73) is found the family G8 does give rise to a Liouvillian solution to equation (9). This solution is given by equation (75):

$$
\eta=\frac{e^{-\frac{s}{2} r}}{r^{\frac{3}{2}}(r-2)^{s-\frac{1}{2}}}(r+\mathrm{k}),
$$

where $s$ and $\mathrm{k}$ are given by equations (82). By combining equations (8) and (83) one obtains the corresponding Liouvillian solution $\eta^{\prime}$ to equation ([6):

$$
\eta^{\prime}=\frac{(r+\mathrm{k})}{r(r-2)^{s}} e^{-\frac{s}{2} r}
$$

The solution $\chi$ found by Chandrasekhar (equation ( $(\mathbf{7 6})$ ) is the same as the solution $\eta^{\prime}$. Indeed, by combining equations (76), (179) and (80) we obtain

$$
\chi=2^{2 \sigma_{0}} \frac{\mu^{2} r+6}{r(r-2)^{2 \sigma_{0}}} e^{-\sigma_{0} r} .
$$

On the other hand, from equations (78), (80), (82) and (84) one finds that

$$
\eta^{\prime}=\frac{1}{\mu^{2}} \frac{\mu^{2} r+6}{r(r-2)^{2 \sigma_{0}}} e^{-\sigma_{0} r}
$$

From equations (85) and (86) it follows that the solution $\eta^{\prime}$ is the same as the solution $\chi$; the solution to an homogeneous equation is determined up to an arbitrary multiplicative constant. This completes the proof.

\section{The family G7 gives the Liouvillian solution $\chi \int \frac{\mathrm{d} r_{*}}{\chi^{2}}$}

The significance of this subsection does not stem from the discovery of the Liouvillian solution $\chi \int \frac{\mathrm{d} r_{*}}{\chi^{2}}$; a Liouvillian solution $\chi$ to the master equation (3) was found in the previous subsection and it is well known that a second linearly independent solution to equation (3) is $\chi \int \frac{\mathrm{d} r_{*}}{\chi^{2}}$ which is also Liouvillian. Moreover, both solutions $\chi$ and $\chi \int \frac{\mathrm{d} r_{*}}{\chi^{2}}$ were found by Chandrasekhar a long time ago $\underline{14}$.

It is the proof itself, that the family G7 gives rise to the Liouvillian solution $\chi \int \frac{\mathrm{d} r_{*}}{\chi^{2}}$, and other results which are obtained in the course of the proof that constitute the new findings of this subsection and contribute to its significance. In particular

- It is verified that Kovacic's algorithm may capture the second Liouvillian solution $\eta \int \frac{e^{-\int a}}{\eta^{2}}$ when a Liouvillian solution $\eta$ is identified. So far in the applications of Kovacic's algorithm the search for pairs $\eta$ and $\eta \int \frac{e^{-\int a}}{\eta^{2}}$, which as it is stated in subsections 
$[\mathrm{A}$ and $[\mathrm{B}$ is one of the most difficult aspects of the application of the algorithm, has been neglected (e.g. $\underline{\underline{26}}, \underline{40})$. The cause of this negligence can be traced back to the original article by Kovacio $\underline{18}$.

- The structure and the logic of the proof does not depend on the particular problem at hand, namely the linear perturbations of the Schwarzschild geometry, but it is tailored for identifying Liouvillian pairs $\eta$ and $\eta \int \frac{e^{-\int a}}{\eta^{2}}$. Therefore it is highly likely that analogues and/or generalizations of the proof are going to be useful in the search for pairs of Liouvillian solutions $\eta$ and $\eta \int \frac{e^{-\int a}}{\eta^{2}}$ in subsequent applications of the algorithm; either these applications refer to black hole geometries, or otherwise.

- Equation (87) after a change of the independent variable falls into the confluent Heun class. Therefore in this case Kovacic's algorithm reduces the problem of finding Liouvillian solutions to the RWE equation to the problem of finding polynomial solutions to equation (87) which falls into the confluent Heun class and one may hope that the literature on the solutions of the confluent Heun equation, which is rather extensive (see e.g. $\underline{\underline{46}} \underline{\underline{49}}$ ), may provide some clues or even provide the answer. However the literature is not helpful and does not provide any clues. The polynomial solution we find here (equations (109), (110) and (117)) has not appeared in the literature so far. We find it by combining Kovacic's algorithm with a new approach.

- In general the coefficients of the series solutions of ODEs with rational function coefficients cannot be calculated in "closed-form" when the ODEs lead to three (or four, five...)-term recurrence relations for these coefficients. Equation (87) is constructed by Kovacic's algorithm and plays a prominent role: Polynomial solutions to equation (87) lead to Liouvillian solutions to the RWE equation (6). We find a "closed-form" polynomial solution (equations (109), (110), (117), and (119)) to equation (87) at odds with what generally happens with ODEs which lead to three-term recurrence relations for the coefficients of their series solutions.

- The second Liouvillian solution $\eta \int \frac{e^{-\int a}}{\eta^{2}}$, initially found by Chandrasekhar $\underline{\underline{14}}$, can be expressed via elementary functions for all values of the angular harmonic index $l$, $l=2,3, \ldots$. This result was given for the first time by Araujo and MacCallum $\underline{44}$ in an unpublished work; however, these authors do not give the proof which leads to it. 
This result was not derived by Chandrasekhar $\underline{\underline{14}}$ and it is difficult to imagine how one could obtain it without applying Kovacic's algorithm to the RWE.

- It is a nice and unexpected surprise that we can combine the result (equations (109), (110), (117)) regarding the polynomial solution to equation (87) in this subsection, with the extension of Hautot's results we give in subsection VIIB, and express, in subsection VIIC, the polynomial solution to equation (87) as a finite sum of truncated confluent hypergeometric functions of the first kind (equation (221)), and also express, in subsection VIID, this polynomial as a finite sum of associated Laguerre polynomials (equation (239)). Both sums hold for every value of the angular harmonic index $l$, $l=2,3, \ldots$.

- As a result, in subsection VIIC, we express in equation (222), the second Liouvillian solution $\eta \int \frac{e^{-\int a}}{\eta^{2}}$ as a finite sum of truncated confluent hypergeometric functions multiplied by a factor involving elementary functions, and also, in subsection VIID, we express in equation (251), the second Liouvillian solution $\eta \int \frac{e^{-\int a}}{\eta^{2}}$ as a finite sum of associated Laguerre polynomials multiplied by the same factor of elementary functions as before. It is again difficult to imagine how we could obtain this result without combining the application of Kovacic's algorithm to the RWE equation with the extension of Hautot's results we give in subsection VIIB, .

- Last but not least, the proof we give in this subsection, showing that the family G7 gives rise to the Liouvillian solution $\chi \int \frac{\mathrm{d} r_{*}}{\chi^{2}}$, is an indispensable part of the proof that there are no other Liouvillian solutions to the master equation (66) apart from those found by Chandrasekhar $\underline{14}$. The proof continues in section VIII, The proof we give in this subsection is totally absent in the consideration of the problem by C.H. $\underline{26}$. What is even more curious is that in $\underline{\underline{26}}$ there is no family, in the case of the gravitational perturbations of the Schwarzschild geometry, which gives rise to the Liouvillian solution $\chi \int \frac{\mathrm{d} r_{*}}{\chi^{2}}$; we elaborate more on this issue in subsection VIIIF However, in $\underline{26}$, the family which leads to the Liouvillian solution $\chi$, we examine this family in subsection $\sqrt{\mathrm{BB}}$, does appear. Due to the succinct form of the presentation of the results $\operatorname{in}^{26}$ we have not been able to trace the origin of this discrepancy.

It is not generally true $e^{20}$ that if one of the retained families gives rise to a Liouvillian 
solution $\eta$ to equation (11) then another retained family will give rise to $\eta \int \frac{e^{-\int a}}{\eta^{2}}$. In fact, as it is stated in subsections $[\mathrm{IA}$ and $\mathrm{IB}$, establishing when this holds is one of the most difficult aspects of applying Kovacic's algorithm and it can take considerable amount of time and effort to find the pair(s) of families, if they exist at all, which yield the Liouvillian solutions $\eta$ and $\eta \int \frac{e^{-\int a}}{\eta^{2}}$. We prove now that in the particular problem under consideration

Theorem 2 The family Gr gives rise to the solution $\chi \int \frac{\mathrm{d} r_{*}}{\chi^{2}}$.

Proof In this case, where $\theta(r)$ and $\nu(r)$ are given respectively by equations (66) and (45), with $\beta=-3$, equation (73) reads

$$
\mathrm{P}^{\prime \prime}(r)+\frac{6-2 r-4 r s+r^{2} s}{r(r-2)} \mathrm{P}^{\prime}(r)+\frac{2-l(l+1)+6 s-r s(1+2 s)}{r(r-2)} \mathrm{P}(r)=0 .
$$

The last equation has two regular singular points at $r=0, r=2$, and, one irregular singular point of Poincare rank 1 at $r=\infty$. The roots of the associated indicial equations are the following

$$
\begin{array}{cr}
\text { Singular points } & \text { Roots } \\
r=0 & \rho_{1}=0, \rho_{2}=4, \\
r=2 & \rho_{1}=0, \rho_{2}=2 s, \\
r=\infty & \rho=1+2 s .
\end{array}
$$

One can easily check that if equation (87) admits a polynomial solution at all then this solution will have degree $1+2 s$, which is precisely the degree determined by Kovacic's algorithm. One can easily check that equation (87) cannot admit polynomial solution of zero or first degree. Thus in this proof we will assume hereafter that $2 s$ is an integer which takes values in the set $\{1,2,3, \ldots\}$.

According to Fuchs' Theorem a basis of the space of solutions of equation (87) in the punctured neighbourhood $(0,2) \cup(2,4)$ of 2 , when $\rho_{2}-\rho_{1}=2 s-0=2 s \in Z^{+}, Z^{+}=$ $\{1,2,3, \ldots\}$, is given by

$$
\begin{aligned}
& p_{1}(r)=(r-2)^{2 s} \sum_{\mathrm{n}=0}^{\infty} \mathrm{P}_{\mathrm{n}}(r-2)^{\mathrm{n}}, \quad \mathrm{P}_{0} \neq 0, \quad \text { and } \\
& p_{2}(r)=\sum_{\mathrm{n}=0}^{\infty} \mathrm{a}_{\mathrm{n}}(r-2)^{\mathrm{n}}+\mathrm{C} p_{1} \ln |r-2|, \quad \mathrm{a}_{0} \neq 0 .
\end{aligned}
$$

The constant $\mathrm{C}$ may turn out to be zero. 
A remark regarding the constant $\mathrm{C}$ is here in order. The constant $\mathrm{C}$ may be determined by substituting $p_{1}(r)=(r-2)^{2 s} \sum_{\mathrm{n}=0}^{\infty} \mathrm{P}_{\mathrm{n}}(r-2)^{\mathrm{n}}, \mathrm{P}_{0} \neq 0$, (equation (88)) ) into equation (87), in order to find the coefficients $\mathrm{P}_{\mathrm{n}}$, and then by substituting $p_{2}(r)=\sum_{\mathrm{n}=0}^{\infty} \mathrm{a}_{\mathrm{n}}(r-2)^{\mathrm{n}}+$ $\mathrm{C} p_{1} \ln |r-2|, \mathrm{a}_{0} \neq 0$, (equation (⒐9) ) into equation (87) in order to find the coefficients $\mathrm{a}_{\mathrm{n}}$ and the constant $\mathrm{C}$. The constant $\mathrm{C}$ may turn out to be zero.

Whenever in this paper, power series solutions of the form (88) and (89) are used in the proofs of Lemmas, Corollaries and Theorems, and in these proofs it is stated that "The constant C may turn out to be zero" this will mean that the proof will not necessitate the evaluation of $\mathrm{C}$. If the value of $\mathrm{C}$ is required from the proof, this will be explicitly stated, $\mathrm{C}$ will be evaluated with the method described above, and its value will be given.

By substituting $\mathrm{P}(r)=(r-2)^{\rho} \sum_{\mathrm{n}=0}^{+\infty} \mathrm{P}_{\mathrm{n}}(r-2)^{\mathrm{n}}$ into equation (87), where $\rho=0$ or $\rho=2 s$, we find that the coefficients $\mathrm{P}_{\mathrm{n}}$ satisfy the following three-term recurrence relation

$$
\begin{aligned}
& -s(2-\mathrm{n}-\rho+2 s) \mathrm{P}_{\mathrm{n}-1}+\left(2-l(l+1)+\mathrm{n}^{2}+\mathrm{n}(2 \rho-3)+\rho(\rho-3)+4 s(1-s)\right) \mathrm{P}_{\mathrm{n}} \\
& +2(1+\mathrm{n}+\rho)(1+\mathrm{n}+\rho-2 s) \mathrm{P}_{\mathrm{n}+1}=0, \quad \text { where, } \quad \mathrm{P}_{-1}=0 .
\end{aligned}
$$

We note that when $\rho=2 s$ equation (90) gives the three-term recurrence relation satisfied by the coefficients $\mathrm{P}_{\mathrm{n}}$ of the power series solution $p_{1}(r)=(r-2)^{2 s} \sum_{\mathrm{n}=0}^{\infty} \mathrm{P}_{\mathrm{n}}(r-2)^{\mathrm{n}}, \mathrm{P}_{0} \neq 0$, given by equation (88). When $\rho=0$ equation (90) gives the three-term recurrence relation satisfied by the coefficients $\mathrm{a}_{\mathrm{n}}$ of the solution $p_{2}(r)=\sum_{\mathrm{n}=0}^{\infty} \mathrm{a}_{\mathrm{n}}(r-2)^{\mathrm{n}}+\mathrm{C} p_{1} \ln |r-2|, \mathrm{a}_{0} \neq 0$, given by equation (89), only when $\mathrm{C}=0$. When $\mathrm{C} \neq 0$ the coefficients $\mathrm{a}_{\mathrm{n}}$ of the power series $\sum_{\mathrm{n}=0}^{\infty} \mathrm{a}_{\mathrm{n}}(r-2)^{\mathrm{n}}$ appearing in the solution $p_{2}(r)$, given by equation (89), are determined by substituting $p_{2}(r)$ into equation (87).

From the three-term recurrence relation (90) we easily verify, by examining the system of equations resulting from (90) when $\rho=2 s$ and $\mathrm{n}=0,1,2$, that the series solution $p_{1}$ given by equation (188), can in principle terminate to give a polynomial solution of degree $2 s+1$, but this can only happen when $s=-\frac{l(l-1)(l+1)(l+2)}{6}, l=2,3, \ldots$. Consequently this case is dismissed since the only accepted values of $s$ are $s=2 \sigma_{0}=\frac{l(l-1)(l+1)(l+2)}{6}, l=2,3, \ldots$, (equation (80)).

However, as we easily verify from the three-term recurrence relation (90), by examining the system of equations resulting from (90) when $\rho=0$ and $\mathrm{n}=2 s+1,2 s+2$, the series solution $p_{2}$ given by equation (89), can, in principle, terminate and give a polynomial solution of degree $2 s+1$, when $s=\frac{l(l-1)(l+1)(l+2)}{6}, l=2,3, \ldots$, if $\mathrm{C}$ is zero. 
If this is indeed the case the coefficients $\mathrm{P}_{\mathrm{n}}$ of the polynomial solution $\mathrm{P}(r)=\sum_{\mathrm{n}=0}^{2 s+1} \mathrm{P}_{\mathrm{n}}(r-$ $2)^{\mathrm{n}}$ to equation (87), satisfy the 3 -term recurrence relation

$$
\begin{array}{r}
s(\mathrm{n}-2(s+1)) \mathrm{P}_{\mathrm{n}-1}+(2-l(l+1)+\mathrm{n}(\mathrm{n}-3)-4 s(s-1)) \mathrm{P}_{\mathrm{n}}+2(1+\mathrm{n})(1+\mathrm{n}-2 s) \mathrm{P}_{\mathrm{n}+1}=0 \\
\mathrm{P}_{-1}=0,(91)
\end{array}
$$

which results from the 3 -term recurrence relation (90) when $\rho=0$.

We search now for a polynomial solution $\mathrm{P}(r)$ of degree $1+2 s$ to equation (87). Proceeding with brutal force and trying to find a polynomial solution to equation (87) is not effective because the degree of the polynomial solution sought for the equation (87) is not fixed but it depends on the parameter $s$ which appears in the coefficients of $\mathrm{P}^{\prime}$ and of $\mathrm{P}$ in equation (87). Remarkably equation (87) after a change of the independent variable from $r$ to $z=\frac{r}{2}$ falls into the confluent Heun class. The extensive literature on the solutions of the confluent Heun equation (see e.g. $\underline{46} \underline{-49}$ ) does not provide any clues either for obtaining polynomial solutions to equation (87).

For this reason, we follow instead another approach: We assume that a polynomial solution $\mathrm{P}(r)$ to equation (73) of degree $1+2 s$ exists, i.e., we assume that the family G7 gives rise to a Liouvillian solution $y$. By making use of equations (66) and (75) we find that

$$
y=\frac{\mathrm{P}(r) e^{\frac{s}{2} r}}{r^{\frac{3}{2}}(r-2)^{s-\frac{1}{2}}} .
$$

By combining equations (8) and (92) we find the corresponding Liouvillian solution $y^{\prime}$ to equation (6) :

$$
y^{\prime}=\frac{\mathrm{P}(r) e^{\frac{s}{2} r}}{r(r-2)^{s}} .
$$

We also assume that $y^{\prime}$ is equal to the solution $\chi \int \frac{\mathrm{d} r_{*}}{\chi^{2}}$ given by equation (77) and that $s$ in equation (93) takes the values given by (equation (80)), i.e., we assume that

$$
s=2 \sigma_{0}=\frac{\mu^{2}\left(\mu^{2}+2\right)}{6}=\frac{l(l-1)(l+1)(l+2)}{6},
$$

where $l=2,3, \ldots$. By making use of equations (176), (79), (193) and (94) the last assumption reads

$$
\int \frac{\mathrm{d} r_{*}}{\chi^{2}}=\int \frac{r^{3}(r-2)^{4 \sigma_{0}-1} e^{2 \sigma_{0} r}}{\left(\mu^{2} r+6\right)^{2}}=\frac{\mathrm{P}(r) e^{2 \sigma_{0} r}}{\mu^{2} r+6} .
$$

Equation (95) implies

$$
\left(\mathrm{P}^{\prime}(r)+2 \sigma_{0} \mathrm{P}(r)\right)\left(\mu^{2} r+6\right)-\mu^{2} \mathrm{P}(r)=r^{3}(r-2)^{4 \sigma_{0}-1} .
$$


By making the change of the independent variable $r=w+2$ in equation (96) we obtain

$$
\left(\mathrm{P}^{\prime}(w)+2 \sigma_{0} \mathrm{P}(w)\right)\left(\mu^{2} w+2 \mu^{2}+6\right)-\mu^{2} \mathrm{P}(w)=w^{4 \sigma_{0}-1}(w+2)^{3} .
$$

$\mathrm{P}(w)$ is a polynomial of degree $1+2 s=1+4 \sigma_{0}$. By setting

$$
\mathrm{P}(w)=\sum_{\mathrm{n}=0}^{1+4 \sigma_{0}} \mathrm{P}_{\mathrm{n}} w^{n}
$$

in (97) we find

$$
\begin{array}{r}
\sum_{\mathrm{n}=0}^{1+4 \sigma_{0}}\left\{2 \sigma_{0} \mu^{2} \mathrm{P}_{\mathrm{n}-1}+\left[\mu^{2} \mathrm{n}+2 \sigma_{0}\left(6+2 \mu^{2}\right)-\mu^{2}\right] \mathrm{P}_{\mathrm{n}}+\left(6+2 \mu^{2}\right)(\mathrm{n}+1) \mathrm{P}_{\mathrm{n}+1}\right\} w^{\mathrm{n}}+ \\
2 \sigma_{0} \mu^{2} \mathrm{P}_{4 \sigma_{0}+1} w^{4 \sigma_{0}+2}=8 w^{4 \sigma_{0}-1}+12 w^{4 \sigma_{0}}+6 w^{4 \sigma_{0}+1}+w^{4 \sigma_{0}+2}
\end{array}
$$

where, $\mathrm{P}_{-1}=\mathrm{P}_{4 \sigma_{0}+2}=0$. From equation (99) all the coefficients $\mathrm{P}_{\mathrm{n}}$ can be found.

This derivation of the coefficients $\mathrm{P}_{\mathrm{n}}$ depends on the validity of the initial assumptions. In order to verify the correctness of the result, we derive again the values of the coefficients $\mathrm{P}_{\mathrm{n}}$ by using in addition a theoretical input independent of any initial assumption. In case of agreement, we do expect the coefficients determined by equation (99) to be proportional to the new coefficients, i.e., we expect the following relation to hold

$$
\mathrm{P}_{\mathrm{n}}=c \mathrm{P}_{\mathrm{n}}^{\prime} \quad \forall \quad 0 \leq \mathrm{n} \leq 1+4 \sigma_{0}
$$

where, $\mathrm{P}_{\mathrm{n}}^{\prime}$ are the new coefficients and $c$ is a constant of proportionality. We use the same symbol $\mathrm{P}_{\mathrm{n}}$ for both derivations. This hopefully does not give rise to any confusion.

Now the second derivation of the coefficients $\mathrm{P}_{\mathrm{n}}$ is as follows. The points $r=0$ and $r=2$ are regular singular points of equation (6). By Fuchs' Theorem a basis of the space of solutions of equation (6), in in the punctured neighbourhood $(0,2) \cup(2,4)$ of 2 , is given by

$$
\begin{aligned}
& \psi_{1}=(r-2)^{s} \sum_{\mathrm{n}=0}^{\infty} \mathrm{b}_{\mathrm{n}}(r-2)^{\mathrm{n}}, \quad \mathrm{b}_{0} \neq 0, \quad \text { and }, \\
& \psi_{2}=(r-2)^{-s} \sum_{\mathrm{n}=0}^{\infty} \mathrm{c}_{\mathrm{n}}(r-2)^{\mathrm{n}}+\mathrm{C} \psi_{1} \ln |r-2|, \quad \mathrm{c}_{0} \neq 0 .
\end{aligned}
$$

The constant $\mathrm{C}$ may turn out to be zero, and, $s$ and $-s$ are the roots of the indicial equation associated with the singular point $r=2$. Their difference $s-(-s)=2 s=4 \sigma_{0}$ is a strictly positive integer (equation (94)). 
The two linearly independent solutions $\chi$ and $y^{\prime}$ of equation (6), given by equations (85) and (93) respectively, may be expressed as linear combinations of $\psi_{1}$ and $\psi_{2}$, i.e., there are constants A, B, D, and F, such that

$$
\begin{aligned}
\frac{\left(\mu^{2} r+6\right) e^{-\sigma_{0} r}}{r(r-2)^{2 \sigma_{0}}} & =\mathrm{A} \psi_{1}+\mathrm{B} \psi_{2}, \\
\frac{\mathrm{P}(\mathrm{r}) \mathrm{e}^{\sigma_{0} \mathrm{r}}}{r(r-2)^{2 \sigma_{0}}} & =\mathrm{D} \psi_{1}+\mathrm{F} \psi_{2} .
\end{aligned}
$$

By combining equations (101), (102), (103), and (104), we find that the functions $\left(\mu^{2} w+\right.$ $\left.2 \mu^{2}+6\right) e^{-\sigma_{0} w}$ and $\mathrm{P}(w) e^{\sigma_{0} w}$ have proportional expansions up to order $w^{4 \sigma_{0}-1}$, i.e., we find that

$$
\mathrm{P}(w) \sim\left(\mu^{2} w+2 \mu^{2}+6\right) e^{-2 \sigma_{0} w}, \quad \text { up to order } w^{4 \sigma_{0}-1}
$$

where, in order to simplify notation, we have used the same symbol $\mathrm{P}$ for the functions $\mathrm{P}(r)$ and $\mathrm{p}(w)=\mathrm{P}(w+2)$.

Now

$$
\left(\mu^{2} w+2 \mu^{2}+6\right) \sum_{\mathrm{n}=0}^{\infty} \frac{\left(-2 \sigma_{0} w\right)^{\mathrm{n}}}{\mathrm{n} !}
$$

has $n^{\text {th }}$ coefficient

$$
\frac{\left(-2 \sigma_{0}\right)^{\mathrm{n}-1}}{\mathrm{n} !}\left[\left(\mathrm{n}-4 \sigma_{0}\right) \mu^{2}-12 \sigma_{0}\right],
$$

and therefore equation (105) gives

$$
\mathrm{P}_{\mathrm{n}} \sim \frac{\left(-2 \sigma_{0}\right)^{\mathrm{n}-1}}{\mathrm{n} !}\left[\left(\mathrm{n}-4 \sigma_{0}\right) \mu^{2}-12 \sigma_{0}\right] \quad \text { for } \quad 0 \leq \mathrm{n} \leq 4 \sigma_{0}-1 \text {. }
$$

From equation (106) it follows that

$$
\mathrm{P}_{4 \sigma_{0}-1} \sim \frac{\left(-2 \sigma_{0}\right)^{4 \sigma_{0}-2}}{\left(4 \sigma_{0}-1\right) !}\left(-\mu^{2}-12 \sigma_{0}\right), \quad \mathrm{P}_{4 \sigma_{0}-2} \sim \frac{\left(-2 \sigma_{0}\right)^{4 \sigma_{0}-3}}{\left(4 \sigma_{0}-2\right) !}\left(-2 \mu^{2}-12 \sigma_{0}\right) .
$$

Equation (107) gives

$$
\frac{\mathrm{P}_{4 \sigma_{0}-1}}{\mathrm{P}_{4 \sigma_{0}-2}}=\frac{\sigma_{0}}{1-4 \sigma_{0}} \frac{\mu^{2}+12 \sigma_{0}}{\mu^{2}+6 \sigma_{0}} .
$$

By a straightforward calculation we verify that the set of values of the coefficients $\mathrm{P}_{\mathrm{n}}$ given by equation (106) satisfy equation (99) for all powers up to $w^{4 \sigma_{0}-2}$. This is a partial verification of the validity of our initial assumptions. There was no reason to anticipate that the coefficients given by equation (106) should satisfy equation (199) for all powers up to $w^{4 \sigma_{0}-2}$, unless our initial assumptions are valid. 
To complete the verification we need to show that these values, i.e. the values (106), can give a consistent solution to the four equations which are obtained by equating the coefficients of $w^{4 \sigma_{0}+2}, w^{4 \sigma_{0}+1}, w^{4 \sigma_{0}}$, and $w^{4 \sigma_{0}-1}$, from both sides of (99). By equating the coefficients of $w^{4 \sigma_{0}+2}$ we find

$$
\mathrm{P}_{4 \sigma_{0}+1}=\frac{1}{2 \sigma_{0} \mu^{2}} .
$$

By equating the coefficients of $w^{4 \sigma_{0}+1}$ and by making use of equation (109) we have that

$$
\mathrm{P}_{4 \sigma_{0}}=\frac{\mu^{2}-3}{\sigma_{0} \mu^{4}}
$$

Equating the coefficients of $w^{4 \sigma_{0}}$ and combining equations (109) and (110) yields

$$
\mathrm{P}_{4 \sigma_{0}-1}=\frac{3\left(6 \sigma_{0}-\mu^{2}\right)}{\sigma_{0}^{2} \mu^{6}} .
$$

Finally, by equating the coefficients of $w^{4 \sigma_{0}-1}$ and by making use of equations (110) and (111) we obtain

$$
\mathrm{P}_{4 \sigma_{0}-2}=\frac{3\left(-36 \sigma_{0}^{2}-12 \mu^{2} \sigma_{0}^{2}+12 \mu^{2} \sigma_{0}+4 \mu^{4} \sigma_{0}-\mu^{4}\right)}{\sigma_{0}^{3} \mu^{8}} .
$$

From equations (111) and (112) it follows that

$$
\frac{\mathrm{P}_{4 \sigma_{0}-1}}{\mathrm{P}_{4 \sigma_{0}-2}}=\frac{\left(\mu^{2}-6 \sigma_{0}\right) \sigma_{0} \mu^{2}}{36 \sigma_{0}^{2}+12 \mu^{2} \sigma_{0}^{2}-12 \mu^{2} \sigma_{0}-4 \mu^{4} \sigma_{0}+\mu^{4}} .
$$

The values of the coefficients $\mathrm{P}_{\mathrm{n}}$ given by equation (106) agree with the values given by equations (109), (110), (111), and (112), if and only if the ratio (108) is equal to the ratio (113). It is straightforward to verify that the equality of the two ratios (108) and (113)

$$
\frac{\sigma_{0}}{1-4 \sigma_{0}} \frac{\mu^{2}+12 \sigma_{0}}{\mu^{2}+6 \sigma_{0}}=\frac{\left(\mu^{2}-6 \sigma_{0}\right) \sigma_{0} \mu^{2}}{36 \sigma_{0}^{2}+12 \mu^{2} \sigma_{0}^{2}-12 \mu^{2} \sigma_{0}-4 \mu^{4} \sigma_{0}+\mu^{4}}
$$

holds if and only if

$$
\sigma_{0}=\frac{\mu^{2}\left(\mu^{2}+2\right)}{12}
$$

But this happens to be one of our initial assumptions (equation (94)). This completes the proof.

We adopt the values of the coefficients $\mathrm{P}_{\mathrm{n}}$ determined by the first derivation (equations (99), (108), (109), (110), and (111) ). The values of the coefficient $\mathrm{P}_{4 \sigma_{0}-1}$ given by the two derivations are different (equations (107) and (111)). Their ratio is equal to the costant of proportionality $c$ (cf.equation (100) $)$

$$
c=\frac{3\left(4 \sigma_{0}\right) !\left(\mu^{2}-6 \sigma_{0}\right)}{\left(-2 \sigma_{0}\right)^{4 \sigma_{0}}\left(12 \sigma_{0}+\mu^{2}\right) \sigma_{0} \mu^{2}} .
$$


To make the values of the coefficients $\mathrm{P}_{\mathrm{n}}$ given by equation (106) consistent with the adopted values (equation (99)) we multiply them by the constant of proportionality $c$ given by equation (116). By doing so we obtain

$$
\mathrm{P}_{\mathrm{n}}=\frac{3\left(-2 \sigma_{0}\right)^{\mathrm{n}-4 \sigma_{0}-1}\left(4 \sigma_{0}\right) !\left(\mu^{2}-6 \sigma_{0}\right)\left[\left(\mathrm{n}-4 \sigma_{0}\right) \mu^{2}-12 \sigma_{0}\right]}{\mathrm{n} !\left(\mu^{2}+12 \sigma_{0}\right) \sigma_{0} \mu^{6}}, \quad 0 \leq \mathrm{n} \leq 4 \sigma_{0}-1 .
$$

Equations (109), (110) and (117) give all the coefficients $\mathrm{P}_{\mathrm{n}}, 0 \leq \mathrm{n} \leq 1+4 \sigma_{0}$, of the polynomial $\mathrm{P}(w)$. As expected, these coefficients also satisfy the 3 -term recurrence relation (91), when $s=2 \sigma_{0}=\frac{l(l-1)(l+1)(l+2)}{6}$.

$\mathrm{P}(w)$ is a polynomial of degree $1+4 \sigma_{0}$ in $w=r-2$ related to the polynomial $\mathrm{P}(r)$ appearing in the numerator of the solution $y^{\prime}$ (equation (93)) by the relation

$$
\mathrm{P}(r)=\sum_{\mathrm{n}=0}^{1+4 \sigma_{0}} \mathrm{p}_{\mathrm{n}} r^{\mathrm{n}}=\sum_{\mathrm{n}=0}^{1+4 \sigma_{0}} \mathrm{P}_{\mathrm{n}}(r-2)^{\mathrm{n}}
$$

where $\mathrm{P}_{\mathrm{n}}$ are given by equations (109), (110) and (117). From equation (118) one can find the coefficients $\mathrm{p}_{\mathrm{n}}$ by using Newton's binomial formula to expand $(r-2)^{\mathrm{n}}$. We easily find

$$
\mathrm{p}_{\mathrm{n}}=\sum_{i=0}^{\left(1+4 \sigma_{0}\right)-\mathrm{n}} \mathrm{P}_{\mathrm{n}+i}\left(\begin{array}{c}
\mathrm{n}+i \\
i
\end{array}\right)(-2)^{i}, \quad 0 \leq \mathrm{n} \leq 1+4 \sigma_{0} .
$$

This completes the proof.

\section{D. $\quad \chi \int \frac{\mathrm{d} r_{*}}{\chi^{2}}$ has an elementary functions answer for all $l$}

The integral $\int \frac{\mathrm{d} r_{*}}{\chi^{2}}$ in the solution $\chi \int \frac{\mathrm{d} r_{*}}{\chi^{2}}$ (see equation (77)) was left unevaluated by Chandrasekhar. Equation (95) shows that it has an elementary function answer for all $l$. For example, for $l=2$ we have $\mu^{2}=4$ and $\sigma_{0}=2$ (equations (78) and (800) and by combining equations (195), (109), (110), (117), and (118), we find

$$
\begin{array}{r}
\int \frac{\mathrm{d} r_{*}}{\chi^{2}}=\int \frac{r^{3}(r-2)^{7} e^{4 r}}{(4 r+6)^{2}} \mathrm{~d} r=\frac{\mathrm{P}(r) e^{4 r}}{4 r+6}= \\
\left(-\frac{1164765}{16384}+\frac{1941275}{8192} r-\frac{388255}{1024} r^{2}+\frac{388255}{1024} r^{3}-\frac{132149}{512} r^{4}+\right. \\
\left.\frac{31345}{256} r^{5}-40 r^{6}+\frac{275}{32} r^{7}-\frac{35}{32} r^{8}+\frac{1}{16} r^{9}\right) \frac{e^{4 r}}{4 r+6} .
\end{array}
$$




\section{HAUTOT'S RESULTS AND PERTURBATIONS OF THE SCHWARZSCHILD GEOMETRY}

In 1969 Hautot wrote a paper $\underline{\underline{60}}$ on the polynomial solutions of the differential equations of the form

$$
z(z-1) \mathrm{P}^{\prime \prime}(z)+\left(a z^{2}+b z+c\right) \mathrm{P}^{\prime}(z)+\left(d+e z+f z^{2}\right) \mathrm{P}(z)=0 .
$$

Kovacic's algorithm key result, stated in subsection IA and in section IV is that there are Liouvillian solutions to the master equation (6) if and only if there are polynomial solutions to equation (73) which reads

$$
\mathrm{P}^{\prime \prime}+2 \theta \mathrm{P}^{\prime}+\left(\theta^{2}+\theta^{\prime}-\nu\right) \mathrm{P}=0
$$

. Equation (121) is of interest to us because in the problem we are examining, in all cases, apart from a few cases examined in section IV and a case examined in subsection $\mathrm{VB}$, equation (73), as it is shown in subsection $\mathrm{VC}$ and in section VIII, falls into the class of equations (121).

Hautot starts his paper by finding the necessary and sufficient conditions for the existence of polynomial solutions to equation (121). These conditions amount to the requirement that the determinant of a tridiagonal matrix, the matrix of the linear homogeneous system associated with the differential equation (121), must be zero. The elements of this determinant are the coefficients of the three-term recurrence relation which results from equation (121) when the polynomial solution $\mathrm{P}_{\mathrm{n}}(z)=\sum_{k=0}^{\mathrm{n}} \lambda_{k} z^{k}$ of degree $\mathrm{n}$ is substituted into it.

Hautot proceeds to point out that the key difficulty in satisfying this requirement is that the order of the determinant increases with the order of the polynomial solution. In fact it is precisely this difficulty which has deterred up to this day the derivation of all the polynomial solutions to equation (121) despite the numerous efforts (e.g. $\underline{\underline{46}} \underline{\underline{49}}$ ).

Hautot then makes a crucial remark upon which his whole paper is based: The key difficulty can be circumvented at the expense of not obtaining the whole set of polynomial solutions but a subset of them by arriving at sufficient, but not necessary, conditions for the existence of polynomial solutions to equation (121).

These new sufficient conditions reduce to the requirement that the determinant of a fixed order $(j+1) \times(j+1)$, the fixed number $j$ being determined by equation (121), must be 
zero. So now one is only faced with the easy task of checking if a determinant of fixed order is zero. If it is, and if in addition, as the relevant analysis shows, $f=0$ and $e=-a$, then equation (121) admits polynomial solutions of degree $n$.

Hautot manages to obtain the necessary conditions by using the structure of the three-term recurrence relation which results from equation (121) and an elementary fact about determinants which one can find in any textbook on linear algebra! Hautot's work is a nice reminder that significant results can be obtained with elementary methods. What adds more to the significance of Hautot's results is that Hautot carries on and proves that when the sufficient conditions are satisfied, i.e., when the aforementioned determinant of fixed order is zero, then the resulting polynomial solutions of degree $n$ can be written as a sum of $j+1$ truncated confluent hypergeometric functions of the first kind!

\section{A. Hautot's results and Liouvillian solutions}

What makes Hautot's results relevant to our analysis is that the sufficient conditions derived by Hautot for the existence of polynomial solutions to equation (121) are met precisely in the case of the Liouvillian solution $\chi \int \frac{\mathrm{d} r_{*}}{\chi^{2}}$, initially found by Chandrasekhar $\frac{14}{4}$, in the case of the gravitational perturbations of the Schwarzschild geometry. In fact Hautot's sufficient

conditions in the case of the Liouvillian solution $\chi \int \frac{\mathrm{d} r_{*}}{\chi^{2}}$ reduce to the requirement that the frequency of the gravitational perturbations can only take the values of the frequencies of the algebraically special gravitational perturbations of the Schwarzschild geometry initially found by Couch and Newman 16 !

After the application, in section [V], of Kovacic's algorithm to the master equation (6), and the examination of the families G8 and G7 in subsections $\mathrm{VB}$ and $\mathrm{VC}$ respectively, there remain four families, namely the families G3, E3, E7, and S3.

In section VIII the four remaining families G3, E3, E7, and S3 are reduced into two families G3 and E7. There is strong evidence, given in subsection VIIID, that the two families G3 and E7 do not give rise to Liouvillian solutions, but a proof of this is still missing.

More to the evidence given in section VIII adds the fact that Hautot's sufficient conditions are not satisfied in cases G3, E3, and S3 and therefore no Liouvillian solutions arise in these cases from Hautot's sufficient conditions. 
Moreover, in the case E7, which arises in the electromagnetic perturbations of the Schwarzschild geometry, Hautot's sufficient conditions are satisfied but they give rise to Liouvillian solutions only when the angular harmonic index $l$ takes the value 0 . This solution describes the first order perturbation of the Schwarzschild geometry due to the presence of a point charge located at the center of the mass-source of the gravitational field, and it is interesting in its own right. However, it does not describe gravitational waves travelling on the Schwarzschild background and as such it is dismissed.

Hautot's sufficient conditions are derived for and are tailored to equation (121) which appears in the application of Kovacic's algorithm to the Schwarzschild geometry in the form of equation (73). In section $\mathrm{VC}$ we prove that the application of Kovacic's algorithm to the Schwarzschild geometry leads to the result that Chandrasekhar's Liouvillian solution $\chi \int \frac{\mathrm{d} r_{*}}{\chi^{2}}$, in the case of the gravitational perturbations of the Schwarzschild geometry, is a product of elementary functions, one of them being a polynomial of degree $2 s+1$, where $s=2 \mathrm{i} \sigma$ and $\sigma$ is the frequency of the monochromatic waves to which the perturbation of the Schwarzschild geometry is Fourier decomposed.

By using an appropriate extension of Hautot's results, which we derive in subsection VIIB, we prove in subsection VIIC that this polynomial, of degree $2 s+1$, admits a finite expansion in terms of truncated confluent hypergeometric functions of the first kind (equation (221) ). Moreover in subsection VIID, by using the same extension of Hautot's results, we prove that this polynomial, of degree $2 s+1$, admits also a finite expansion in terms of associated Laguerre polynomials (equation (239) $)$. Remarkably, both expansions hold for every value of the angular harmonic index $l, l=2,3, \ldots$.

We expect that Hautot's analysis, appropriately modified and extended, is applicable to other black-hole geometries, 4-dim and higher, and will help, in conjunction with Kovacic's algorithm, to find Liouvillian solutions to the perturbation equations of these other geometries, and express them in certain cases, as finite sums of truncated (confluent) hypergeometric functions.

A modification of Hautot's results is necessary in order to apply them to other black hole geometries, because in other geometries Kovacic's algorithm will lead in general to equations which will not fall into the class (121), and an extension of Hautot's results is needed, for the reasons explained in subsection VIIB, in order to apply them to the Schwarzschild geometry in 4-dim, and also in order to apply them, to other black-hole geometries, 4-dim and 
higher.

With this motivation we present Hautot's results $\$ \underline{60}$ emphasizing those points which are more relevant to our analysis and highlighting the underlying reasoning. This will make more intelligible

1. The necessary modification of Hautot's results, in order to apply them to other black hole geometries.

2. A rectification made in subsection VID2 of an oversight by Hautot.

3. More importantly, the necessary extension of Hautot's results, introduced in subsection VIIB, in order to apply them to the problem under consideration.

Most likely a similar extension is needed in order to apply Hautot's results to other black hole geometries.

\section{B. Necessary and sufficient conditions}

By assuming that equation (121) admits a polynomial solution $\mathrm{P}_{\mathrm{n}}(z)=\sum_{k=0}^{\mathrm{n}} \lambda_{k} z^{k}$ of degree $\mathrm{n}$ and by substituting $\mathrm{P}_{\mathrm{n}}(z)$ into equation (121) we obtain $f=0, e=-a \mathrm{n}$, and that the coefficients $\lambda_{k}, k=0,1,2, \ldots$, satisfy the three-term recurrence relation

$$
a(k-1-\mathrm{n}) \lambda_{k-1}+(d+k(b+k-1)) \lambda_{k}+(c-k)(k+1) \lambda_{k+1}=0 .
$$

If equation (121) admits polynomial solutions $\mathrm{P}_{\mathrm{n}}(z)$ then the determinant of the associated linear homogeneous system $(\mathrm{n}+1) \times(\mathrm{n}+1)$ is zero.

Let us denote this determinant by $\operatorname{det}(\mathcal{M})$, where $\mathcal{M}$ is the $(n+1) \times(n+1)$ matrix of the coefficients of the $\mathrm{n}+1$ unknowns $\lambda_{0}, \lambda_{1}, \lambda_{2}, \ldots, \lambda_{\mathrm{n}+1}$ of this linear homogeneous system. The determinant $\operatorname{det}(\mathcal{M})$ is tridiagonal and it involves the coefficients

$$
R_{k}=a(k-1-\mathrm{n}), \quad S_{k}=d+k(b+k-1), \quad T_{k}=(c-k)(k+1),
$$

$k=0,1,2, \ldots, \mathrm{n}$, of the three-term recurrence relation (122).

We conclude that the necessary conditions for the existence of polynomial solutions to equation (121) are:

1.

$$
f=0
$$


2.

$$
e=-a \mathrm{n}
$$

3.

$$
\operatorname{det}(\mathcal{M})=\left|\begin{array}{ccccccc}
S_{0} & T_{0} & 0 & \cdots & 0 & 0 & 0 \\
R_{1} & S_{1} & T_{1} & \cdots & 0 & 0 & 0 \\
\cdot & \cdot & \cdot & \cdots & \cdot & \cdot & \cdot \\
\cdot & \cdot & \cdot & \cdots & \cdot & \cdot & \cdot \\
\cdot & \cdot & \cdot & \cdots & \cdot & \cdot & \cdot \\
\cdot & \cdot & \cdot & \cdots & \cdot & \cdot & \cdot \\
0 & \cdot & \cdot & \cdots & R_{\mathrm{n}-1} & S_{\mathrm{n}-1} & T_{\mathrm{n}-1} \\
0 & \cdot & \cdot & \cdots & \cdot & R_{\mathrm{n}} & S_{\mathrm{n}}
\end{array}\right|=0
$$

Conditions 1, 2, and 3, are also sufficient for the existence of polynomial solutions to equation (121). As pointed out by Hautot $\underline{60}$ the key difficulty in satisfying condition 3 is that the order of the determinant increases with the order $n$ of the polynomial solution.

This difficulty, as expected, appears not only when we try to solve the linear homogeneous system associated with the differential equation $\underline{\underline{60}}$ but also when we try to find polynomial solutions to equation (121) with any of the standard methods, eg., by studying the structure of the three-term recurrence relation (122), by considering the continued fraction associated to the three-term recurrence relation (122), by expanding the solution of (121) in terms of confluent hypergeometric functions and the like $e^{48} \underline{\underline{49}}$, etc..

When $f=0$ equations (173) and (121) fall into the confluent Heun class. It is worth noting that there is a monograph on the Heun's differential equation and its various confluent forms ${ }^{54}$, and on page 118 of this monograph we read that the necessary and sufficient conditions for the existence of polynomial solutions to equation (121) are the aforementioned conditions 1,2 , and 3 .

Some of the subsequent work on the exact solutions of the Regge-Wheeler equation, see e.g. $\underline{30}, 31$, was based on this monograph and on the conditions 1, 2, and 3 which were also stated there. As a result, there is no overlap between that work and this work which is not based on the conditions 1,2 , and 3 , but it is based instead on the sufficient conditions found by Hautot $\underline{\underline{60}}$ which are stated in the next subsection. 


\section{Hautot's sufficient conditions and $\chi \int \frac{d r_{*}}{\chi^{2}}$}

Hautot's sufficient conditions are based on the structure of the three-term recurrence relation (122) and on an elementary fact about determinants which one can find in any introductory textbook on linear algebra.

If $\mathcal{C}$ is a square matrix of order $m+q$ and has the form

$$
\left(\begin{array}{ll}
\mathcal{A} & \mathrm{O} \\
\mathcal{D} & \mathcal{B}
\end{array}\right),
$$

where $\mathcal{A}$ and $\mathcal{B}$ are square matrices of order $m$ and $q$ respectively, $\mathbf{O}$ is a $m \times q$ zero matrix, and $\mathcal{D}$ is a $q \times m$ matrix, then

$$
\operatorname{det}(\mathcal{C})=\operatorname{det}(\mathcal{A}) \cdot \operatorname{det}(\mathcal{B})
$$

where, $\operatorname{det}(\mathcal{C}), \operatorname{det}(\mathcal{A})$, and $\operatorname{det}(\mathcal{B})$, denote respectively the determinants of the matrices $\mathcal{C}$, $\mathcal{A}$, and $\mathcal{B}$.

The crucial remark made by Hautot is that if $c=j(=0,1,2, \ldots$, fixed $)$ then the coefficient $T_{j}$ of the three-term recurrence relation (122) vanishes, and the square matrix $\mathcal{M}$ of order

$\mathrm{n}+1$, whose determinant appears in condition 3 , takes the form of the matrix $\mathcal{C}$, i.e., when $c=j(=0,1,2, \ldots$, fixed $)$, we have

$$
\mathcal{M}=\left(\begin{array}{ll}
\mathcal{A} & \mathrm{O} \\
\mathcal{D} & \mathcal{B}
\end{array}\right) .
$$

In this case $\mathcal{A}, \mathcal{B}$ are square matrices of order $j+1$ and $\mathrm{n}-j$ respectively, $\mathbf{O}$ is a $(j+1) \times$ $(\mathrm{n}-j)$ zero matrix, and $\mathcal{D}$ is a $(\mathrm{n}-j) \times(j+1)$ matrix.

The matrices $\mathcal{A}, \mathcal{B}$ and $\mathcal{D}$ are given explicitly by

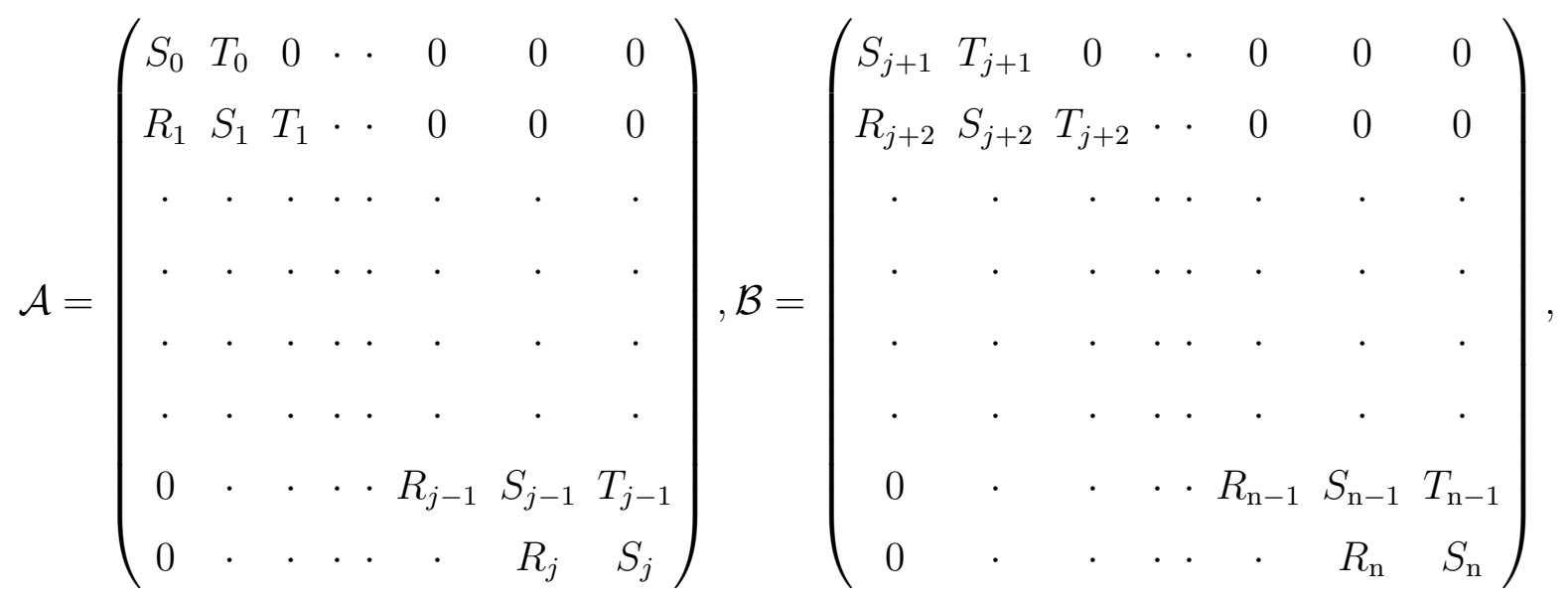


and

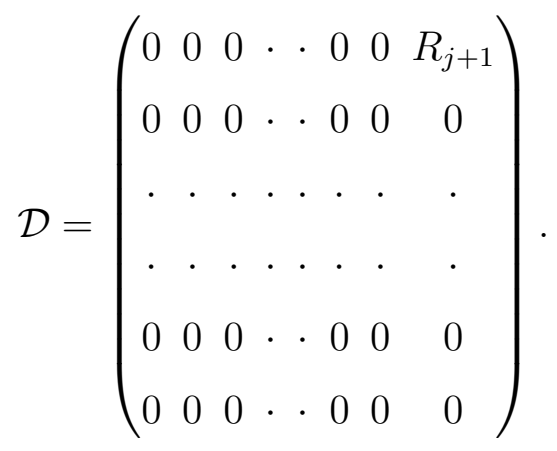

In accordance with equation (128)

$$
\operatorname{det}(\mathcal{M})=\operatorname{det}(\mathcal{A}) \cdot \operatorname{det}(\mathcal{B})
$$

where $\operatorname{det}(\mathcal{M})$ is given in equation $(\underline{126})$, and $(\mathcal{A}),(\mathcal{B})$ are given in equation (130).

Equation (132), Hautot notices, suggests sufficient conditions for obtaining polynomial solutions to equation (121). In fact there are two possibilities:

1st possibility: $\operatorname{det}(\mathcal{A})=0$, and consequently, $\operatorname{det}(\mathcal{M})=0$ and as a result equation (121) has polynomial solutions.

2nd possibility: $\operatorname{det}(\mathcal{B})=0$, and consequently, $\operatorname{det}(\mathcal{M})=0$ and as a result equation (121) has polynomial solutions.

A few remarks are now in order

- In the second possibility it is assumed that $\operatorname{det}(\mathcal{A}) \neq 0$, and therefore it is assumed that $\lambda_{1}=\lambda_{2}=\ldots=\lambda_{j}=0$.

- It is difficult in general to pursue the second possibility further because $\operatorname{det}(\mathcal{B})$ shares the same main feature with $\operatorname{det}(\mathcal{M})$ : the order of $\operatorname{det}(\mathcal{B})$ increases with the order of the polynomial solution to equation (121); so we restrict our attention to the first possibility.

- In the case of the Liouvillian solution $\chi \int \frac{\mathrm{d} r_{*}}{\chi^{2}}$ found by Chandrasekhar, the first possibility reduces to the requirement that the frequency of the gravitational perturbations can only take the values of the frequencies of the algebraically special gravitational perturbations of the Schwarzschild geometry initially found by Couch and Newman 16 ! 
- The difficulty in obtaining the whole set of polynomial solutions to equation (121) is brought again to the fore: Even in the special case where $c=j$ it is difficult to obtain all the polynomial solutions to equation (121) since there are in general such solutions which arise from the second possibility which cannot in general be pursued further.

Hautot concludes that if we dismiss the second possibility, since it is difficult to be pursued further, and keep only the first possibility we arrive at the following sufficient conditions for the existence of polynomial solutions to equation (121):

1.

$$
f=0
$$

2.

$$
e=-a \mathrm{n}
$$

3.

$$
c=j(=0,1,2, \ldots, \text { fixed })
$$

4.

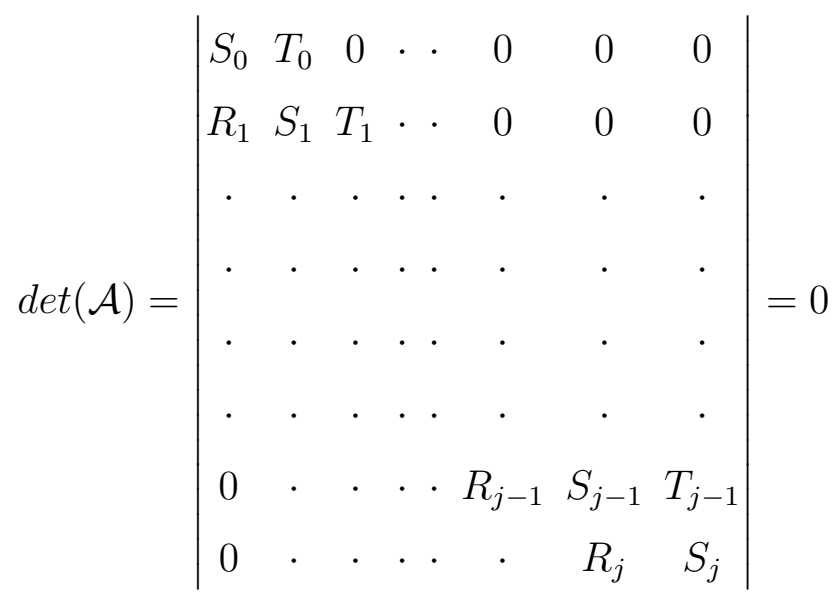

Hautot's sufficient conditions, when satisfied, do not give the whole set of polynomial solutions to equation (121), even in the special case $c=j$, since in general there are polynomial solutions to equation (121) which arise from the second possibility, $\operatorname{det}(\mathcal{B})=0$, which is difficult in general to be pursued further. To put it differently, Hautot's sufficient conditions are not necessary, precisely because there are in general polynomial solutions to equation (121) resulting from the second possibility, $\operatorname{det}(\mathcal{B})=0$.

In subsection $\mathrm{VC}$ we proved that the family $\mathrm{G} 7$ gives rise to the Liouvillian solution $\chi \int \frac{\mathrm{d} r_{*}}{\chi^{2}}$, found by Chandrasekhar ${ }^{14}$, which describes algebraically special perturbations of the 
Schwarzschild geometry which excite only incoming or only outgoing waves. The frequencies $\sigma$ of these algebraically special perturbations can only take values in a denumerable infinite $\operatorname{set}^{14,16}$ (equation (800)

$$
\mathrm{i} \sigma=\frac{l(l-1)(l+1)(l+2)}{12}, \quad l=2,3, \ldots
$$

In the case of the family G7, $\theta(r)$ and $\nu(r)$ are given respectively by (67) and (45), with $\beta=-3$, and equation (73) reads (equation(

$$
r(r-2) \mathrm{P}^{\prime \prime}(r)+\left(6-2 r-4 r s+r^{2} s\right) \mathrm{P}^{\prime}(r)+(2-l(l+1)+6 s-r s(1+2 s)) \mathrm{P}(r)=0,
$$

where $s=2 \mathrm{i} \sigma$. With a change of the independent variable from $r$ to $z=\frac{r}{2}$ the previous equation becomes

$$
z(z-1) \mathrm{P}^{\prime \prime}(z)+\left(2 s z^{2}-2(2 s+1) z+3\right) \mathrm{P}^{\prime}(z)+(-2 s(2 s+1) z+2-l(l+1)+6 s) \mathrm{P}(z)=0,
$$

where now the differentiation is understood with respect to $z$.

This equation, which falls into the confluent Heun class, has the same form as equation (121) which is the object of study of Hautot's paper ${ }^{60}$ with

$$
\begin{aligned}
& f=0, \\
& e=-a \text { n }=-2 s(2 s+1), \text { and } \\
& c=3 .
\end{aligned}
$$

Moreover, the requirement

$$
\operatorname{det}(\mathcal{A})=\left|\begin{array}{cccc}
S_{0} & T_{0} & 0 & 0 \\
R_{1} & S_{1} & T_{1} & 0 \\
0 & R_{2} & S_{2} & T_{2} \\
0 & 0 & R_{3} & S_{3}
\end{array}\right|=0
$$

where the coefficients $R_{k}, S_{k}$, and $\mathrm{T}_{k}, k=0,1,2,3$, are the coefficients of the three-term recurrence relation (122) and are given by equation (123), reads

$$
\operatorname{det}(\mathcal{A})=\left|\begin{array}{cccc}
2-l(l+1)+6 s & 3 & 0 & 0 \\
-2 s(2 s+1) & 2 s-l(l+1) & 4 & 0 \\
0 & -4 s^{2} & -2 s-l(l+1) & 3 \\
0 & 0 & 2 s(1-2 s) & 2-6 s-l(l+1)
\end{array}\right|=0 .
$$


Requirement (142) is equivalent to

$$
s= \pm \frac{l(l-1)(l+1)(l+2)}{6} \Leftrightarrow \mathrm{i} \sigma= \pm \frac{l(l-1)(l+1)(l+2)}{12}, \quad l=2,3, \ldots
$$

which are precisely the frequencies $\frac{14,16}{16}$ of the algebraically special perturbations of the Schwarzschild geometry! The frequencies in equation (143) appear with + or - sign. To restore the ambiguity regarding the sign we note that so far the frequencies of the algebraically special perturbations appear with the $+\operatorname{sign}($ equation (80)) because of the form of the time dependence $\exp [-\mathrm{i} \sigma t]$ we have assumed in the Fourier decomposition of the perturbing field $\Phi(t, r, \theta, \phi)$ at the beginning of our study, equation (2); perturbations which become infinite as $t \rightarrow+\infty$ are not physically acceptable.

Hautot's sufficient conditions (133), (134), (135), and (136), when satisfied, lead to a

polynomial solution $\mathrm{P}_{\mathrm{n}}(z)=\sum_{k=0}^{\mathrm{n}} \lambda_{k} z^{k}$ of degree $\mathrm{n}$ to equation (121). The coefficients of this polynomial solution satisfy the three-term recurrence relation (122). Hautot carries on and proves that when these sufficient conditions are satisfied, the polynomial solution to equation (121), resulting from the sufficient conditions, can be expressed in "closed-form" as a finite sum of truncated confluent hypergeometric functions of the first kind. This is the content of subsection VID.

In subsection $\mathrm{VC}$, in the case of Chandrasekhar's Liouvillian solution $\chi \int \frac{\mathrm{d} r_{*}}{\chi^{2}}$, we found in "closed-form" the polynomial solution to the ODE (87), associated to the family G7. As we said before, equation (87), after a change of the independent variable from $r$ to $z=\frac{r}{2}$, becomes equation (137) which falls into the confluent Heun class and has the same form as equation (121). This suggests that the "closed-form" polynomial solution, given by equations (109), (110) and (117), we found to the ODE (87) admits an expansion in terms of truncated confluent hypergeometric functions of the first kind.

In subsection we prove that this is precisely the case. However the proof involves an unexpected twist: Hautot's results need to be extended in order to prove that the polynomial solution to the ODE (121) admits such an expansion. We give this extension in subsection VIIB.

\section{Polynomial solutions to the confluent Heun equation}

Hautot's sufficient conditions (133), (134), (135), and (136), when satisfied, ensure the existence of polynomial solutions to equation (121). The coefficients of these solutions satisfy 
the three-term recurrence relation (122). In general solutions to three-term recurrence relations cannot be expressed in "closed-form". Remarkably, Hautot $\underline{60}$ proved that the polynomial solutions to equation (121) ensuing from the sufficient conditions he found, can be expressed in "closed-form" as a finite sum of truncated confluent hypergeometric functions.

This is an unexpected result and constitutes the most significant part of Hautot's nice paper ${ }^{60}$. We note in passing that several confluent hypergeometric expansions of the solutions of the confluent Heun equation have been considered recently in $\stackrel{48}{ }$. These expansions differ from the expansion given by Hautot which is more appropriate for our purposes.

The crucial remark which underlies Hautot's proof is the following. When Hautot's sufficient conditions (133), (134), (135), and (136) are satisfied then equation (121), where $f=0, e=-a$ n, and $c=j$, admits polynomial solution $\mathrm{P}_{\mathrm{n}}(z)=\sum_{k=0}^{\mathrm{n}} \lambda_{k} z^{k}$ of degree $\mathrm{n}$, and therefore yields

$$
z(z-1) \mathrm{P}_{\mathrm{n}}^{\prime \prime}(z)+\left(a z^{2}+b z+j\right) \mathrm{P}_{\mathrm{n}}^{\prime}(z)+(d-a \mathrm{n} z) \mathrm{P}_{\mathrm{n}}(z)=0
$$

This equation, falls into the confluent Heun class and has two regular singular points at $z=0$ and at $z=1$ and one irregular singular point at $z=\infty$. Möbius transformations of the independent variable play a central role in the study of linear ODEs with rational function coefficients (see e.g. ${ }^{56.78}$ ). As expected prominent role have the transformations which interchange and/or shift the locations of the singular points.

Under the linear transformation

$$
u=a(1-z)
$$

of the independent variable $z$ equation (144) becomes

$$
(u-a)\left(u \mathrm{P}_{\mathrm{n}}^{\prime \prime}(u)+((a+b+j)-u) \mathrm{P}_{\mathrm{n}}^{\prime}(u)+\mathrm{nP}_{\mathrm{n}}(u)\right)-u j \mathrm{P}_{\mathrm{n}}^{\prime}(u)+d \mathrm{P}_{\mathrm{n}}(u)=0
$$

where for simplicity we use the same symbol $\mathrm{P}_{\mathrm{n}}(\mathrm{u})$ for the polynomial of degree $\mathrm{n}, \mathrm{P}_{\mathrm{n}}\left(\frac{a-u}{a}\right)$.

\section{Polynomial solutions in terms of truncated confluent hypergeometric functions of the first kind}

Regarding equation (146) we note that equation

$$
u y^{\prime \prime}(u)+(q-u) y^{\prime}(u)-e y(u)=0
$$


is the confluent hypergeometric equation. This equation has a regular singular point at $u=0$ and an irregular singular point at $u=\infty$. The roots $\rho_{1}, \rho_{2}$ of the indicial equation associated to the regular singular point $u=0$, are $\rho_{1}=0$ and $\rho_{2}=1-q$.

For future reference we note the following. According to Fuchs' Theorem a basis of the space of solutions of equation (VIIIA) in the neighbourhood $|u|<\infty$ of $u=0$ depends on $q$, and, when $q$ is not an integer is given by

$$
y_{1}(u)=\sum_{k=0}^{\infty} \frac{(e)_{k}}{(q)_{k}} \frac{u^{k}}{k !} \equiv F(e, q ; u) \quad \text { and } \quad y_{2}(u)=u^{1-q} F(e+1-q, 2-q ; u),
$$

when $1-q$ is a positive integer is given by

$$
\begin{aligned}
& y_{1}(u)=u^{1-q} F(e+1-q, 2-q ; u) \text { and } \\
& y_{2}(u)=\sum_{\mathrm{n}=0}^{\infty} \mathrm{d}_{\mathrm{n}} u^{\mathrm{n}}+\mathrm{C} y_{1}(u) \ln |u|, \text { where } \mathrm{C} \text { may turn out to be zero, }
\end{aligned}
$$

and finally, when $q=1$, is given by

$$
y_{1}(u)=F(e, 1 ; u) \quad \text { and } \quad y_{2}(u)=\sum_{\mathrm{n}=0}^{\infty} \mathrm{e}_{\mathrm{n}} u^{\mathrm{n}}+y_{1}(u) \ln |u| .
$$

It is the second case, when 1-q is a positive integer, which is more relevant in subsection VIIB.

The symbol $(w)_{k}$, is the Pochammer's symbol, and is defined by

$$
(w)_{k}=w(w+1) \ldots(w+k-1)
$$

The function $F(e, q ; u)$ is called confluent hypergeometric function of the first kind or Kummer's function of the first kind. When $e=-\mathrm{n}$, $\mathrm{n}$ being a natural number, $F(e, q ; u)$ is truncated and it reduces to a polynomial $\mathrm{P}_{\mathrm{n}}(u)=F(-\mathrm{n}, q ; u)$ of degree $\mathrm{n}$

$$
\mathrm{P}_{\mathrm{n}}(u)=F(-\mathrm{n}, q ; u)=1-\frac{\mathrm{n}}{q} u+\frac{\mathrm{n}(\mathrm{n}-1)}{q(q+1)} \frac{u^{2}}{2 !}+\ldots+\frac{(-1)^{\mathrm{n}} \mathrm{n} !}{q(q+1) \ldots(q+\mathrm{n}-1)} \frac{u^{\mathrm{n}}}{\mathrm{n} !} .
$$

Obviously equation (146) is not satisfied by $\mathrm{P}_{\mathrm{n}}(u)=F(-\mathrm{n}, a+b+j ; u)$. However the possibility arises that equation (146) is satisfied by a linear combination of truncated confluent hypergeometric functions. With this motivation Hautot ${ }^{60}$ starts with the ansatz

$$
\mathrm{P}_{\mathrm{n}}(u)=\sum_{k=0}^{j} A_{k} F(k-\mathrm{n}, a+b+j ; u)
$$


By following Hautot we substitute $P_{\mathrm{n}}(u)$ into equation (146) with this sum of truncated confluent hypergeometric functions of the first kind and we obtain

$$
\begin{aligned}
& (u-a)\left(\sum_{k=0}^{j} A_{k}\left(u F^{\prime \prime}(k-\mathrm{n})+((a+b+j)-u) F^{\prime}(k-\mathrm{n})+(\mathrm{n}-k) F(k-\mathrm{n})\right)\right) \\
& +\sum_{k=0}^{j} A_{k}\left(-u j F^{\prime}(k-\mathrm{n})+(d-a k) F(k-\mathrm{n})+u k F(k-\mathrm{n})\right)=0
\end{aligned}
$$

where for simplicity we write $F(k-\mathrm{n})$ instead of $F(k-\mathrm{n}, a+b+j ; u)$; the differentiation is with respect to $u$. The polynomial $F(k-\mathrm{n})$ satisfies the confluent hypergeometric equation $u F^{\prime \prime}(k-\mathrm{n})+((a+b+j)-u) F^{\prime}(k-\mathrm{n})+(\mathrm{n}-k) F(k-\mathrm{n})=0$ and therefore the last equation yields

$$
\sum_{k=0}^{j} A_{k}\left(-u j F^{\prime}(k-\mathrm{n})+(d-a k) F(k-\mathrm{n})+u k F(k-\mathrm{n})\right)=0 .
$$

By using the recurrence relations

$$
\begin{aligned}
u F^{\prime}(k-\mathrm{n}) & =(\mathrm{n}-k) F(k-\mathrm{n})+(k-\mathrm{n}) \mathrm{F}(k-\mathrm{n}+1), \\
u F(k-\mathrm{n}) & =(k-\mathrm{n}) \mathrm{F}(k-\mathrm{n}+1)-(2(k-\mathrm{n})-(a+b+j)) F(k-\mathrm{n}) \\
& -(a+b+j-(k-\mathrm{n})) \mathrm{F}(k-\mathrm{n}-1)
\end{aligned}
$$

satisfied by the confluent hypergeometric functions of the first $\operatorname{kind} F(k-\mathrm{n})$ (see e.g. ${ }^{79} \mathrm{p}$. 506, 507), equation (156) yields

$$
\begin{aligned}
& \sum_{k=0}^{j}\left\{(k-1-j)(k-1-\mathrm{n}) A_{k-1}+(d-j \mathrm{n}+k(b+2 j-2 k+2 \mathrm{n})) A_{k}\right. \\
& \left.\quad+(k+1)(k+1-\mathrm{n}-(a+b+j)) A_{k+1}\right\} F(k-\mathrm{n})=0 .
\end{aligned}
$$

The last condition can be satisfied for non zero values of the coefficients $A_{k}, k=0,1, \ldots, j$, if and only if

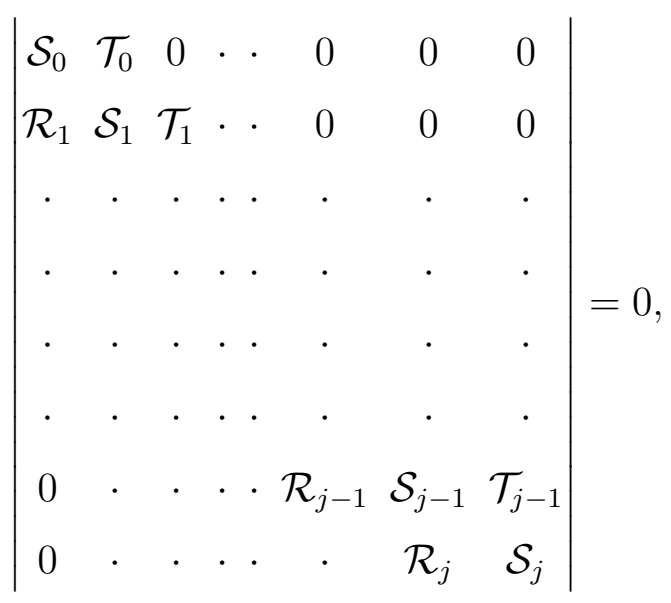


where

$$
\begin{aligned}
\mathcal{R}_{k} & =(k-1-j)(k-1-\mathrm{n}), \\
\mathcal{S}_{k} & =d-j \mathrm{n}+k(b+2 j-2 k+2 \mathrm{n}), \text { and }, \\
\mathcal{T}_{k} & =(k+1)(k+1-\mathrm{n}-a-b-j) .
\end{aligned}
$$

We conclude that when condition (160) is satisfied then equation (146), and therefore equation (144), admits a polynomial solution of degree n. Therefore condition (160) is equivalent to condition (136) . Indeed, it can be verified that for every value of $j=0,1,2, \ldots$, the following equation holds

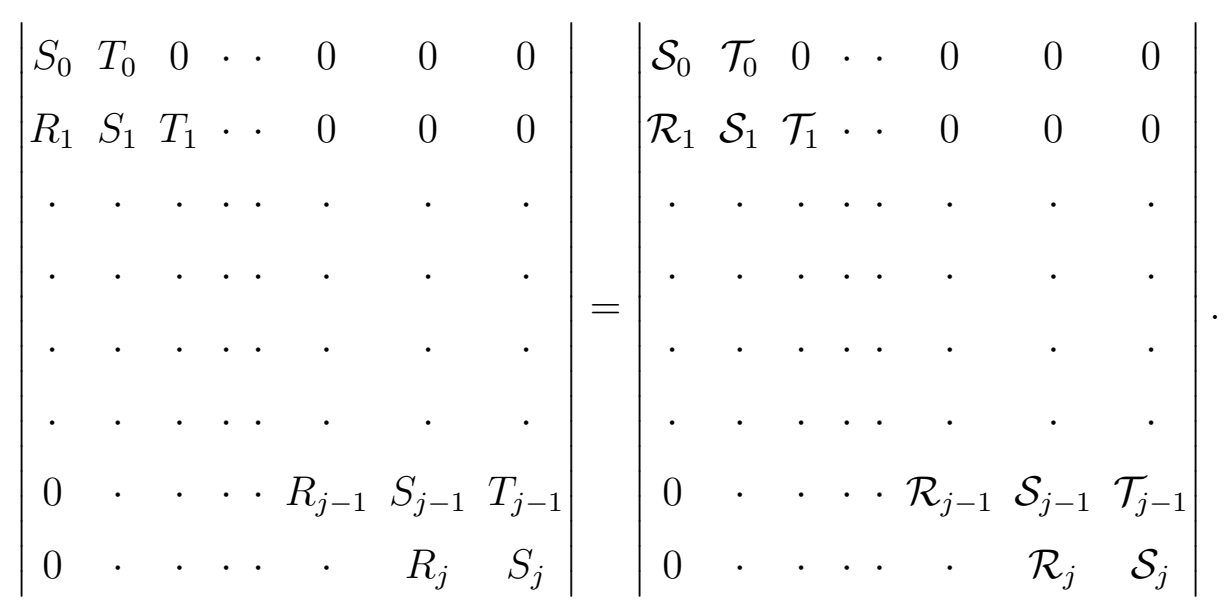

The coefficients $A_{k}, k=0,1, \ldots, j$, in the expansion $\mathrm{P}_{\mathrm{n}}(u)=\sum_{k=0}^{j} A_{k} F(k-\mathrm{n}, a+b+j ; u)$, are determined up to a multiplicative constant, since the polynomial $\mathrm{P}_{\mathrm{n}}(u)$ satisfies the linear homogeneous equation (146).

Two remarks are here in order

- The crucial element in Hautot's derivation is the following: The Polynomial $P_{\mathrm{n}}(u)$ satisfies equation (146), an equation with three singular points. The previous analysis shows that $P_{\mathrm{n}}(u)$ admits an expansion in terms of a finite sum of truncated confluent hypergeometric functions of the first kind which satisfy the confluent hypergeometric equation, an equation with two singular points. As Hautot points out $\frac{60}{}$ it seems that the number of singularities has been diminished by one. This phenomenon is not accidental. Hautot does not give an explanation of it $\underline{60} \underline{-63}$. To the best of our knowledge the first systematic study and explanation of this phenomenon was given by Craster and Shanin in $\underline{64}$. Interestingly enough, a recent study ${ }^{65}$ gives the prerequisites for a a 
group theoretical explanation of this phenomenon. We elaborate more on this issue in section IX,

- We give in some detail Hautot's derivation because in his paper it is incorrectly stated that expansion of the Polynomial solution $P_{\mathrm{n}}(u)$ to equation (146) into a finite sum of Laguerre polynomials leads to equation (312) and subsequently to condition (160). As the previous analysis shows equation (312) and subsequently condition (160) are derived starting from the ansatz that the Polynomial solution $P_{\mathrm{n}}(u)$ admits an expansion in terms of a finite sum of truncated confluent hypergeometric functions of the first kindand not in terms of Laguerre polynomials.

\section{Polynomial solutions in terms of associated Laguerre polynomials}

True the previous analysis can be repeated by expanding $P_{\mathrm{n}}(u)$ into a finite sum of associated Laguerre polynomials but then we are not led to equation (312) and subsequently to condition (160) as Hautot claims 60 .

Indeed starting with the ansatz

$$
\mathrm{P}_{\mathrm{n}}(u)=\sum_{k=0}^{j} B_{k} L_{\mathrm{n}-k}^{(a+b+j-1)}(u)
$$

where $L_{\mathrm{n}-k}^{(a+b+j-1)}(u)$ is the associated Laguerre polynomial which satisfies the equation

$$
u y^{\prime \prime}(u)+(a+b+j-u) y^{\prime}(u)+(\mathrm{n}-k) y(u)=0
$$

and substituting into equation (146) we obtain

$$
\sum_{k=0}^{j} B_{k}\left(-u j L_{\mathrm{n}-k}^{\prime(a+b+j-1)}(u)+(d-a k) L_{\mathrm{n}-k}^{(a+b+j-1)}(u)+u k L_{\mathrm{n}-k}^{(a+b+j-1)}(u)\right)=0 .
$$

The associated Laguerre polynomials $L_{\mathrm{n}-k}^{(a+b+j-1)}(u)$ satisfy the recurrence relations

$$
\begin{aligned}
u L_{\mathrm{n}-k}^{(a+b+j-1)}(u) & =(\mathrm{n}-k) L_{\mathrm{n}-k}^{(a+b+j-1)}(u)-(\mathrm{n}-k+a+b+j-1) \mathrm{L}_{\mathrm{n}-k}^{(a+b+j-1)}(\mathrm{u}) \\
u L_{\mathrm{n}-k}^{(a+b+j-1)}(u) & =-(\mathrm{n}-k+a+b+j-1) \mathrm{L}_{\mathrm{n}-k-1}^{(a+b+j-1)}(\mathrm{u}) \\
& +(2(\mathrm{n}-k)+a+b+j) L_{\mathrm{n}-k}^{(a+b+j-1)}(u) \\
& -(\mathrm{n}-k+1) \mathrm{L}_{\mathrm{n}-k+1}^{(a+b+j-1)}(\mathrm{u}) .
\end{aligned}
$$


We note that associated Laguerre polynomials $L_{\mathrm{n}-k}^{(a+b+j-1)}(u)$ and confluent hypergeometric functions $F(k-\mathrm{n}, a+b+j, u)$ are related via

$$
L_{\mathrm{n}-k}^{(a+b+j-1)}(u)=\left(\begin{array}{c}
\mathrm{n}-k+a+b+j-1 \\
\mathrm{n}-k
\end{array}\right) F(k-\mathrm{n}, a+b+j, u),
$$

where

$$
\left(\begin{array}{l}
N \\
K
\end{array}\right)=\frac{N(N-1)(N-2) \cdots(N-K+1)}{K !}
$$

is the binomial coefficient.

The recurrence relations (168) and (169) can be derived from the recurrence relations (157) and (158) by using the relation (170). With the use of the recurrence relations (168) and (169) equation (313) yields

$$
\begin{aligned}
& \sum_{k=0}^{j}\left\{(k-1-j)(k-\mathrm{n}-a-b-j) B_{k-1}+(d-j \mathrm{n}+k(b+2 j-2 k+2 \mathrm{n})) B_{k}\right. \\
& \left.\quad+(k+1)(k-\mathrm{n}) B_{k+1}\right\} L_{\mathrm{n}-k}^{(a+b+j-1)}(u)=0 .
\end{aligned}
$$

The last condition can be satisfied for non zero values of the coefficients $B_{k}, k=0,1, \ldots, j$, if and only if

$$
\left|\begin{array}{ccccccc}
\mathcal{S}_{0} & \mathcal{W}_{0} & 0 & \cdots & 0 & 0 & 0 \\
\mathcal{Q}_{1} & \mathcal{S}_{1} & \mathcal{W}_{1} & \cdots & 0 & 0 & 0 \\
\cdot & \cdot & \cdot & \cdot & \cdot & \cdot & \cdot \\
\cdot & \cdot & \cdot & \cdot & \cdot & \cdot & \cdot \\
\cdot & \cdot & \cdot & \cdots & \cdot & \cdot & \cdot \\
\cdot & \cdot & \cdot & \cdots & \cdot & \cdot & \cdot \\
0 & \cdot & \cdot & \cdot & \mathcal{Q}_{j-1} & \mathcal{S}_{j-1} & \mathcal{W}_{j-1} \\
0 & \cdot & \cdot & \cdot & \cdot & \mathcal{Q}_{j} & \mathcal{S}_{j}
\end{array}\right|=0
$$

where

$$
\begin{aligned}
\mathcal{Q}_{k} & =(k-1-j)(k-\mathrm{n}-a-b-j), \\
\mathcal{S}_{k} & =d-j \mathrm{n}+k(b+2 j-2 k+2 \mathrm{n}), \text { and, } \\
\mathcal{W}_{k} & =(k+1)(k-\mathrm{n}) .
\end{aligned}
$$

We conclude that when condition (304) is satisfied then equation (146), and therefore equation (144), admits a polynomial solution of degree n. Therefore condition (304) is 
equivalent to condition (136). As in the case of confluent hypergeometric functions, it can be verified that for every value of $j=0,1,2, \ldots$, the following equation holds

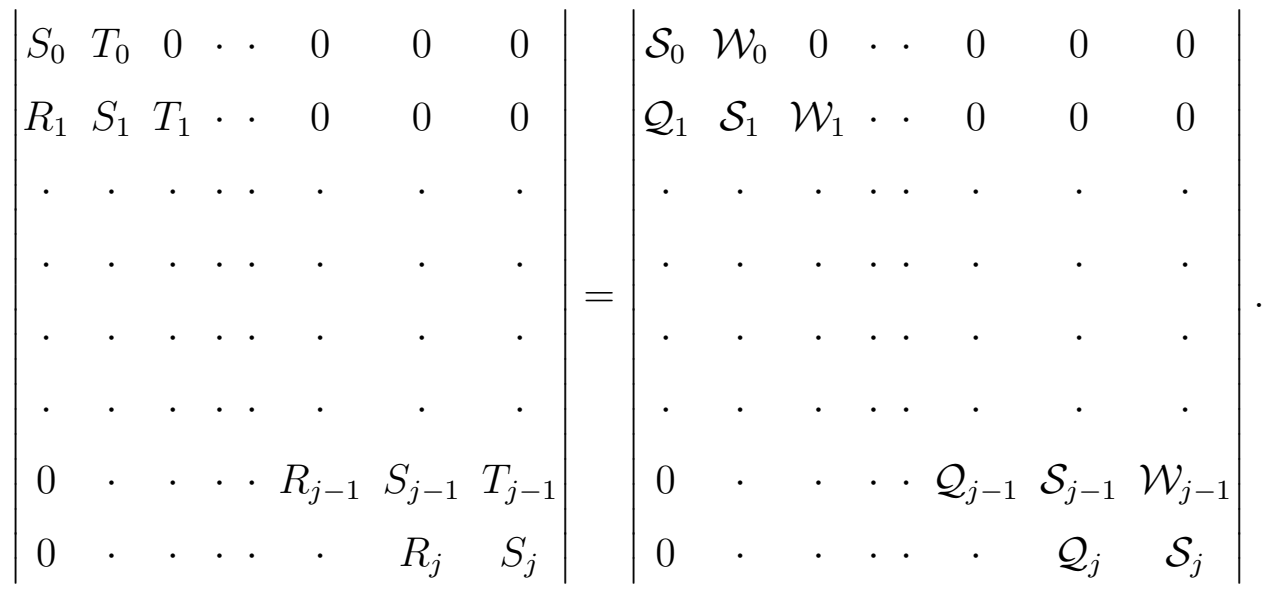

The coefficients $B_{k}, k=0,1, \ldots, j$, in the expansion $\mathrm{P}_{\mathrm{n}}(u)=\sum_{k=0}^{j} B_{k} L_{\mathrm{n}-k}^{(a+b+j-1)}(u)$, are determined up to a multiplicative constant, since $\mathrm{P}_{\mathrm{n}}(u)$ satisfies the linear homogeneous equation (146).

To summarize we conclude that equation (144), $z(z-1) \mathrm{P}_{\mathrm{n}}^{\prime \prime}(z)+\left(a z^{2}+b z+j\right) \mathrm{P}_{\mathrm{n}}^{\prime}(z)+$ $(d-a \mathrm{n} z) \mathrm{P}_{\mathrm{n}}(z)=0$, when Hautot's sufficient condition (136) is satisfied, admits polynomial solution $\mathrm{P}_{\mathrm{n}}(z)$ of degree $\mathrm{n}$, which when it is written as a polynomial in the variable $u=a(1-$ z) admits an expansion both in terms of a finite sum of truncated confluent hypergeometric functions of the first kindand in terms of a finite sum of associated Laguerre polynomials.

\section{VII. $\quad \chi \int \frac{\mathrm{d} r_{*}}{\chi^{2}}$ IN TERMS OF SPECIAL FUNCTIONS}

In subsection $\mathrm{VC}$ using Kovacic's algorithm we showed that Chandrasekhar's Liouvillian solution $\chi \int \frac{\mathrm{d} r_{*}}{\chi^{2}}$ can be put into "closed-form" which involves a polynomial solution of equation (87) which we were also able to give in "closed-form". Equation (87) after a change of the independent variable from $r$ to $z=\frac{r}{2}$ falls into the confluent Heun class, and in particular it falls into the form (144).

This fact provides the link between the results of subsection $\mathrm{VC}$ and the results of the subsections VID 1 and VID2. As a result we expect to be able to express the polynomial solution of equation (87), we found in section $\mathrm{VC}$, in terms of a finite sum of truncated confluent hypergeometric functions of the first kindin the form given in subsection VID 1 or in terms of a finite sum of associated Laguerre polynomials in the form given in subsection VID 2. 
To our surprise when we try to verify that the "closed-form" polynomial solution to equation (87) admits such an expansion, we encounter an obstruction, since, as we show in subsection VIIB, two of the four terms of the sum are not defined in the case of the Liouvillian solution $\eta \int \frac{e^{-\int a}}{\eta^{2}}$ initially found by Chanadrasekhar ${ }^{14}$.

So it appears, that oddly enough, although in the case of the the Liouvillian solution $\eta \int \frac{e^{-\int a}}{\eta^{2}}$, initially found by Chanadrasekhar $\stackrel{14}{ }$, Hautot's sufficient conditions are satisfied, Hautot's polynomial solution to the associated confluent Heun equation does not arise in this case.

We resolve this apparent paradox in subsections VIIB, VIIC and VIID, where we introduce an extension of Hautot's results $\underline{60}^{-63}$, and use this extension in order to prove that the confluent Heun equation, associated with Chandrasekhar's Liouvillian solution $\eta \int \frac{e^{-\int a}}{\eta^{2}}$, does admit polynomial solution which can be expressed both as a sum of truncated confluent hypergeometric functions of the first kind and as a sum of associated Laguerre polynomials, with appropriate function coefficients in each case. Both sums hold for every value of the angular harmonic index $l=2,3, \ldots$.

\section{A. $\chi \int \frac{\mathrm{d} r_{*}}{\chi^{2}}$ and Hautot's results}

In subsection $\mathrm{VC}$ we showed that in the case of the gravitational perturbations of the Schwarzschild geometry the family G7 gives rise to a liouvillian solution to the equation (6) governing the perturbations of the Schwarzschild geometry, namely to the solution $\chi \int \frac{\mathrm{d} r_{*}}{\chi^{2}}$ initially found by Chandrasekhar ${ }^{14}$.

In particular we showed that this solution is equal to (equation (93))

$$
y^{\prime}=\frac{\mathrm{P}(r) e^{\frac{s}{2} r}}{r(r-2)^{s}},
$$

where $\mathrm{P}(\mathrm{r})$ is a polynomial solution to equation (87)

$$
r(r-2) \mathrm{P}^{\prime \prime}(r)+\left(6-2 r-4 r s+r^{2} s\right) \mathrm{P}^{\prime}(r)+(2-l(l+1)+6 s-r s(1+2 s)) \mathrm{P}(r)=0 .
$$

With the change of the independent variable $r=w+2$ the last equation reads

$w(w+2) \mathrm{P}^{\prime \prime}(w)+\left(2-2 w+s\left(-4+w^{2}\right)\right) \mathrm{P}^{\prime}(w)+(2-l(l+1)+6 s-(w+2) s(1+2 s)) \mathrm{P}(w)=0$. 
In subsection $\mathrm{VC}$ we proved that the last equation admits polynomial solutions $\mathrm{P}(w)=$ $\sum_{\mathrm{n}=0}^{1+4 \sigma_{0}} \mathrm{P}_{\mathrm{n}} w^{\mathrm{n}}$ of degree $1+4 \sigma_{0}$ where $\mathrm{P}_{\mathrm{n}}$ are given by equations (109), (110), and (117)

$$
\begin{aligned}
\mathrm{P}_{\mathrm{n}} & =\frac{3\left(-2 \sigma_{0}\right)^{\mathrm{n}-4 \sigma_{0}-1}\left(4 \sigma_{0}\right) !\left(\mu^{2}-6 \sigma_{0}\right)\left[\left(\mathrm{n}-4 \sigma_{0}\right) \mu^{2}-12 \sigma_{0}\right]}{\mathrm{n} !\left(\mu^{2}+12 \sigma_{0}\right) \sigma_{0} \mu^{6}}, \quad 0 \leq \mathrm{n} \leq 4 \sigma_{0}-1, \\
\mathrm{P}_{4 \sigma_{0}} & =\frac{\mu^{2}-3}{\sigma_{0} \mu^{4}}, \quad \mathrm{P}_{4 \sigma_{0}+1}=\frac{1}{2 \sigma_{0} \mu^{2}},
\end{aligned}
$$

where $\mu^{2}=(l-1)(l+2)$ (equation $(\underline{78})$ ) and $s=2 \sigma_{0}=\frac{l(l-1)(l+1)(l+2)}{6}, \quad l=2,3, \ldots$.

Equation (87) after a change of the independent variable from $r$ to $z=\frac{r}{2}$ falls into the confluent Heun class, in particular it falls into the form (144), and it becomes equation (137) which reads

$z(z-1) \mathrm{P}^{\prime \prime}(z)+\left(2 s z^{2}-2(2 s+1) z+3\right) \mathrm{P}^{\prime}(z)+(-2 s(2 s+1) z+2-l(l+1)+6 s) \mathrm{P}(z)=0$.

This fact provides the link between the results of subsection $\mathrm{VC}$ and the results of the subsections VID 1 and VID 2, With a new change of the independent variable from $z$ to $u=2 s(1-z)$ equation (137) falls into the form (146) and in particular it becomes

$u(u-2 s) \mathrm{P}^{\prime \prime}(u)+\left(-u^{2}-2 u-2 s(-2 s+1)\right) \mathrm{P}^{\prime}(u)+((2 s+1) u+2-l(l+1)+4 s(1-s)) \mathrm{P}(u)=0$.

The change of the independent variable $w$ to

$$
u=-s w
$$

transforms equation (178) into equation (179).

We conclude that we can restate what we proved in subsection $\mathrm{VC}$ as follows: Equation (179) has polynomial solution $\mathrm{P}(u)=\sum_{\mathrm{n}=0}^{1+2 s} P_{\mathrm{n}} u^{\mathrm{n}}$ of degree $1+2 s$ with coefficients $P_{\mathrm{n}}$ given by

$$
\begin{aligned}
P_{\mathrm{n}} & =-\frac{6 s^{-2 s-1}(2 s) !((l-1)(l+2)-3 s)[(\mathrm{n}-2 s)(l-1)(l+2)-6 s]}{\mathrm{n} !((l-1)(l+2)+6 s) s(l-1)^{3}(l+2)^{3}}, \\
P_{2 s} & =\frac{2(l-1)(l+2)-6}{s^{2 s+1}(l-1)^{2}(l+2)^{2}}, \quad P_{2 s+1}=-\frac{1}{s^{2 s+2}(l-1)(l+2)},
\end{aligned}
$$

where $0 \leq \mathrm{n} \leq 2 s-1$ and $s=\frac{l(l-1)(l+1)(l+2)}{6}, \quad l=2,3, \ldots$.

We note that the coefficients $P_{\mathrm{n}}, 0 \leq \mathrm{n} \leq 2 s+1$, are related to the coefficients $\mathrm{P}_{\mathrm{n}}$, $0 \leq \mathrm{n} \leq 2 s+1$, given by equations (109), (110), and (117), of the polynomial solution $\mathrm{P}(w)=\sum_{\mathrm{n}=0}^{1+4 \sigma_{0}} \mathrm{P}_{\mathrm{n}} w^{\mathrm{n}}$, by

$$
P_{\mathrm{n}}=\frac{\mathrm{P}_{\mathrm{n}}}{(-s)^{\mathrm{n}}}, \quad 0 \leq \mathrm{n} \leq 2 s+1
$$


As explained at the end of subsection VIC and in subsection VID 1 Hautot proved that when $s=\frac{l(l-1)(l+1)(l+2)}{6}, \quad l=2,3, \ldots$, equation (179) has polynomial solution $\mathrm{P}_{2 \mathrm{~s}+1}(u)$ of degree $2 s+1$ which admits an expansion in terms of a finite sum of truncated confluent hypergeometric functions of the first kind. By comparing equations (137) and (144) we obtain the values of the parameters $a, b, j, \mathrm{n}$, and $d$,

$$
a=2 s, b=-2(1+2 s), j=3, \mathrm{n}=2 s+1, d=2-l(l+1)+6 s .
$$

This expansion is given in the general case by equation (154) which now (equation (184)) reads

$$
\mathrm{P}_{2 s+1}(u)=\sum_{k=0}^{3} A_{k} F(k-(2 s+1), 1-2 s ; u)
$$

The constants $A_{k}$ are determined, up to an arbitrary multiplicative constant, as solutions to the $4 \times 4$ linear homogeneous system resulting from equation (312).

Thus we expect that the polynomial solution $\mathrm{P}(u)$ of degree $2 s+1$ we found in subsection $\mathrm{VC}$ to equation (179), given by equations (181) and (182), admits expansion of the form (185) in terms of truncated confluent hypergeometric functions of the first kind. This brings us to the next subsection.

\section{B. Extension of Hautot's results}

As soon as we try to apply Hautot's results and form the sum $\sum_{k=0}^{3} A_{k} F(k-(2 s+1), 1-$ $2 s ; u)$ we encounter an obstruction since $F(k-(2 s+1), 1-2 s ; u)$ is not defined when $k=0$ and when $k=1$. So it appears, that oddly enough, although in the case of the the Liouvillian solution $\eta \int \frac{e^{-\int a}}{\eta^{2}}$, initially found by Chanadrasekhar $\stackrel{14}{ }$, Hautot's sufficient conditions are satisfied, Hautot's polynomial solution to the associated confluent Heun equation does not arise in this case.

In order to resolve this apparent paradox we need to appropriately extend Hautot's results $\underline{60} \underline{-63}$ and use this extension in order to prove that the confluent Heun equation, associated with Chandrasekhar's Liouvillian solution $\eta \int \frac{e^{-\int a}}{\eta^{2}}$, does admit polynomial solution which can be expressed as a sum of truncated confluent hypergeometric functions of the first kind. This is the content of the following Theorem.

Theorem 3 Equation (179)

$u(u-2 s) \mathrm{P}^{\prime \prime}(u)+\left(-u^{2}-2 u-2 s(-2 s+1)\right) \mathrm{P}^{\prime}(u)+((2 s+1) u+2-l(l+1)+4 s(1-s)) \mathrm{P}(u)=0$ 
admits a polynomial solution

$$
\mathrm{P}_{2 s+1}(u)=\sum_{k=0}^{1} A_{k} \phi((2 s+1)-k ; u)+\sum_{k=2}^{3} A_{k} F(k-(2 s+1), 1-2 s ; u)
$$

of degree $2 s+1$, if and only if

$$
s= \pm \frac{l(l-1)(l+1)(l+2)}{6}
$$

where

$$
\begin{aligned}
\phi(2 s+1 ; u) & =u^{2 s} F(-1,2 s+1 ; u)=u^{2 s}\left(1-\frac{1}{2 s+1} u\right), \\
\phi(2 s ; u) & =u^{2 s} F(0,2 s+1 ; u)=u^{2 s} \\
F(-(2 s-1), 1-2 s ; u) & =1+u+\frac{u^{2}}{2 !}+\frac{u^{3}}{3 !}+\ldots+\frac{u^{2 s-1}}{(2 s-1) !} \\
F(-(2 s-2), 1-2 s ; u) & =1+\frac{2-2 s}{1-2 s} u+\frac{3-2 s}{1-2 s} \frac{u^{2}}{2 !}+\ldots+\frac{(-1)}{1-2 s} \frac{u^{2 s-2}}{(2 s-2) !}
\end{aligned}
$$

and where,

$$
A_{1}=-\frac{l^{2}+l+1}{2 s+1} A_{0}, \quad A_{2}=-\frac{3(2 s) !}{2 s+1} A_{0}, \quad A_{3}=-\frac{\left(l^{2}+l-3\right)(2 s) !}{2 s+1} A_{0},
$$

$A_{0}$ is arbitrary.

\section{Proof}

The confluent Heun equation associated with the family G7, and consequently associated with Chandrasekhar's Liouvillian solution $\eta \int \frac{e^{-\int a}}{\eta^{2}}$, is equation (137) which reads $z(z-1) \mathrm{P}^{\prime \prime}(z)+\left(2 s z^{2}-2(2 s+1) z+3\right) \mathrm{P}^{\prime}(z)+(-2 s(2 s+1) z+2-l(l+1)+6 s) \mathrm{P}(z)=0$.

As we stated in subsection VIIA with a new change of the independent variable from $z$ to $u=2 s(1-z)$ equation (137) becomes equation (179) which reads

$u(u-2 s) \mathrm{P}^{\prime \prime}(u)+\left(-u^{2}-2 u-2 s(-2 s+1)\right) \mathrm{P}^{\prime}(u)+((2 s+1) u+2-l(l+1)+4 s(1-s)) \mathrm{P}(u)=0$.

We rewrite the last equation as follows (see equations (144) and (146))

$$
(u-2 s)\left(u \mathrm{P}^{\prime \prime}(u)+(1-2 s-u) \mathrm{P}^{\prime}(u)+(2 s+1) \mathrm{P}(u)\right)-3 u \mathrm{P}^{\prime}(u)+(2-l(l+1)+6 s) \mathrm{P}(u)=0 .
$$

Of crucial importance is the solution space of the confluent hypergeometric equation (equation (VIIIA)

$$
u y^{\prime \prime}(u)+(1-2 s-u) y^{\prime}(u)-e y(u)=0
$$


$e=k-(2 s+1), k=0,1,2,3$, which appears in equation (186). Strictly speaking in (186) appears only the confluent hypergeometric equation (187) with $k=0$ but the confluent hypergeometric equations with $k=1,2,3$ are also going to be useful in our proof of this Theorem (compare equations (VIIIA), (154), and (155)), so we include them here.

The key observation is that the confluent hypergeometric equation (187) admits a polynomial solution of degree $2 s+1$ when $k=0$ and a polynomial solution of degree $2 s$ when $k=1$. We denote these solutions by $\phi(2 s+1 ; u)$ and $\phi(2 s ; u)$ respectively.

This suggests, as a possible way out to the aforementioned obstruction, to replace $F(-(2 s+1), 1-2 s ; u)$ and $F(-2 s, 1-2 s ; u)$ in the sum $\sum_{k=0}^{3} A_{k} F(k-(2 s+1), 1-2 s ; u)$ by $\phi(2 s+1 ; u)$ and $\phi(2 s ; u)$ respectively. The polynomial solutions $\phi(2 s ; u)$ and $\phi(2 s+1 ; u)$ are not truncated confluent hypergeometric functions of the first kind but they involve in their expressions such functions.

On the other hand the polynomials $F(-(2 s-1), 1-2 s ; u)$ and $F(-(2 s-2), 1-2 s ; u)$ are truncated confluent hypergeometric functions of the first kind defined by equation (272), and are given explicitly by

$$
\begin{aligned}
& F(-(2 s-1), 1-2 s ; u)=1+u+\frac{u^{2}}{2 !}+\frac{u^{3}}{3 !}+\ldots+\frac{u^{2 s-1}}{(2 s-1) !} \\
& F(-(2 s-2), 1-2 s ; u)=1+\frac{2-2 s}{1-2 s} u+\frac{3-2 s}{1-2 s} \frac{u^{2}}{2 !}+\ldots+\frac{(-1)}{1-2 s} \frac{u^{2 s-2}}{(2 s-2) !} .
\end{aligned}
$$

It is useful to examine how the four polynomial solutions $\phi(2 s+1 ; u), \phi(2 s ; u), F(-(2 s-$ $1), 1-2 s ; u), F(-(2 s-2), 1-2 s ; u)$ to the confluent hypergeometric equation $u y^{\prime \prime}(u)+(1-$ $2 s-u) y^{\prime}(u)-e y(u)=0, e=k-(2 s+1), k=0,1,2,3$, result from its two solutions $y_{1}(u)$, and $y_{2}(u)$, given by equations (149) and (150) respectively.

It will thus be made apparent that the confluent hypergeometric equation does not have any other polynomial solutions in the corresponding cases $e=-(2 s+1), e=-2 s, e=$ $-(2 s-1)$, and $e=-(2 s-2)$, and as a result, the uniqueness of the sum $\sum_{k=0}^{3} A_{k} F(k-$ $(2 s+1), 1-2 s ; u)$ will become evident.

We start with the polynomial solutions $\phi(2 s+1 ; u)$ and $\phi(2 s ; u)$. Since $1-q=2 s$ is a positive integer, according to equation (149), the confluent hypergeometric equation admits polynomial solutions when $e=-(2 s+1)(k=0)$ and when $e=-2 s(k=1)$ which are 
given respectively by

$$
\begin{aligned}
\phi(2 s+1 ; u) & =u^{2 s} F(-1,2 s+1 ; u)=u^{2 s}\left(1-\frac{1}{2 s+1} u\right), \text { and } \\
\phi(2 s ; u) & =u^{2 s} F(0,2 s+1 ; u)=u^{2 s} .
\end{aligned}
$$

It is worth mentioning that both polynomial solutions are obtained from the first solution $y_{1}(u)$, given by equation (149), which in both cases terminates to give solutions (190) and (191).

In none of the two cases the corresponding confluent hypergeometric equation admits another polynomial solution since it is easy to check that in neither of the two cases the second linearly independent solution $y_{2}(u)$, given by equation (150), terminates to a polynomial solution; in particular, in neither of the two cases the constant $\mathrm{C}$ which appears in equation (150) is equal to zero.

Thus we have:

When $e=-(2 s+1)(k=0)$,

$$
\begin{aligned}
& y_{1}(u)=\phi(2 s+1 ; u)=u^{2 s} F(-1,2 s+1 ; u)=u^{2 s}\left(1-\frac{1}{2 s+1} u\right), \text { and } \\
& y_{2}(u)=\sum_{\mathrm{n}=0}^{\infty} \mathrm{f}_{\mathrm{n}} u^{\mathrm{n}}+\mathrm{C} y_{1}(u) \ln |u|, \text { where } \mathrm{C} \neq 0 .
\end{aligned}
$$

When $e=-2 s(k=1)$,

$$
\begin{aligned}
& y_{1}(u)=\phi(2 s ; u)=u^{2 s} F(0,2 s+1 ; u)=u^{2 s}, \text { and } \\
& y_{2}(u)=\sum_{\mathrm{n}=0}^{\infty} \mathrm{g}_{\mathrm{n}} u^{\mathrm{n}}+\mathrm{C} y_{1}(u) \ln |u|, \text { where } \mathrm{C} \neq 0 .
\end{aligned}
$$

Moreover, the confluent hypergeometric equation admits polynomial solutions also in the two remaining cases, i.e., when $e=-(2 s-1)(k=2)$, and when $e=-(2 s-2)(k=3)$, which are given respectively by

$$
F(-(2 s-1), 1-2 s ; u) \text { and } F(-(2 s-2), 1-2 s ; u) .
$$

Both of these polynomial solutions result now from the second linearly independent solution $y_{2}(u)$ (equation (150)). 
In particular we have:

When $e=-(2 s-1)(k=2)$,

$$
\begin{aligned}
y_{1}(u)= & u^{2 s} F(1,2 s+1 ; u) \text { which does not terminate, and, } \\
y_{2}(u)= & \sum_{\mathrm{n}=0}^{\infty} \mathrm{h}_{\mathrm{n}} u^{\mathrm{n}}=\mathrm{h}_{0} F(-(2 s-1), 1-2 s ; u)+\mathrm{h}_{2 s} y_{1}(u), \\
& \text { where } \mathrm{h}_{0}, \mathrm{~h}_{2 s} \text { arbitrary and } \mathrm{C}=0 .
\end{aligned}
$$

Finally when $e=-(2 s-2)(k=3)$,

$$
\begin{aligned}
y_{1}(u)= & u^{2 s} F(2,2 s+1 ; u) \text { which does not terminate, and, } \\
y_{2}(u)= & \sum_{\mathrm{n}=0}^{\infty} \mathrm{j}_{\mathrm{n}} u^{\mathrm{n}}=\mathrm{j}_{0} F(-(2 s-2), 1-2 s ; u)+\mathrm{j}_{2 s} y_{1}(u), \\
& \text { where } \mathrm{j}_{0}, \mathrm{j}_{2 s} \text { arbitrary and } \mathrm{C}=0 .
\end{aligned}
$$

We showed how the four polynomial solutions $\phi(2 s+1 ; u), \phi(2 s ; u), F(-(2 s-1), 1-$ $2 s ; u), F(-(2 s-2), 1-2 s ; u)$ to the confluent hypergeometric equation result from its two solutions $y_{1}(u)$ and $y_{2}(u)$, given by equations (149) and (150) respectively. We follow through now Hautot's analysis by substituting $F(-(2 s+1), 1-2 s ; u)$ and $F(-2 s, 1-2 s ; u)$ in the $\operatorname{sum} \sum_{k=0}^{3} A_{k} F(k-(2 s+1), 1-2 s ; u)$ with $\phi(2 s+1 ; u)$ and $\phi(2 s ; u)$ respectively.

We thus substitute

$$
\mathrm{P}_{2 s+1}(u)=\sum_{k=0}^{1} A_{k} \phi((2 s+1)-k ; u)+\sum_{k=2}^{3} A_{k} F(k-(2 s+1), 1-2 s ; u)
$$

into equation (186) and we obtain

$$
\begin{aligned}
& \sum_{k=0}^{1} A_{k}\left(-3 u \phi^{\prime}((2 s+1)-k)+(d-2 s k) \phi((2 s+1)-k)+u k \phi((2 s+1)-k)\right)+ \\
& \sum_{k=2}^{3} A_{k}\left(-3 u F^{\prime}(k-(2 s+1))+(d-2 s k) F(k-(2 s+1))+u k F(k-(2 s+1))\right)=0,(202
\end{aligned}
$$

where $d=2-l(l+1)+6 s$, and where for simplicity, in the rest of this subsection, we write $\phi((2 s+1)-k)$ instead of $\phi((2 s+1)-k ; u)$, and $F(k-(2 s+1))$ instead of $F(k-(2 s+$ 1), $1-2 s ; u)$.

By using appropriate recurrence relations for the four polynomial solutions $\phi(2 s+$ $1), \phi(2 s), F(-(2 s-1)), F(-(2 s-2))$ the last equation yields a $4 \times 4$ homogeneous system for the coefficients $A_{k}, k=0,1,2,3$. We give in turn for the various values of $k$ the appropriate recurrence relations. These are the same with the recurrence relations (157) and (158) only when $k=3$, which, for completeness, we also give explicitly. 
We have, when $k=0$

$$
\begin{aligned}
u \phi^{\prime}(2 s+1) & =(2 s+1) \phi(2 s+1)-\phi(2 s), \\
u \phi(2 s+1) & =-\phi(2 s)+(2 s+3) \phi(2 s+1)-(2 s+2) \phi(2 s+2),
\end{aligned}
$$

when $k=1$

$$
\begin{aligned}
u \phi^{\prime}(2 s) & =2 s \phi(2 s), \\
u \phi(2 s) & =(2 s+1) \phi(2 s)-(2 s+1) \phi(2 s+1),
\end{aligned}
$$

when $k=2$

$$
\begin{aligned}
u F^{\prime}(-(2 s-1)) & =(2 s-1) F(-(2 s-1))-(2 s-1) F(-(2 s-2)) \\
u F(-(2 s-1)) & =-(2 s-1) F(-(2 s-2))-(1-2 s) F(-(2 s-1))+ \\
& +\frac{1}{(2 s-1) !} \phi(2 s), \text { and }
\end{aligned}
$$

when $k=3$,

$$
\begin{aligned}
u F^{\prime}(-(2 s-2)) & =(2 s-2) F(-(2 s-2))-(2 s-2) F(-(2 s-3)) \\
u F(-(2 s-2)) & =-(2 s-2) F(-(2 s-3))+(2 s-3) F(-(2 s-2))+ \\
& +F(-(2 s-1))
\end{aligned}
$$

By substituting recurrence relations (203) - (210) into equation (202) we obtain the following $4 \times 4$ homogeneous system for the coefficients $A_{k}, k=0,1,2,3$,

$$
\begin{array}{r}
-\left(1+l+l^{2}\right) A_{0}-(2 s+1) A_{1}=0 \\
3 A_{0}+(3-l(l+1)) A_{1}+\frac{2}{(2 s-1) !} A_{2}=0 \\
(3-l(l+1)) A_{2}+3 A_{3}=0 \\
(2 s-1) A_{2}+(-1-l(l+1)) A_{3}=0 .
\end{array}
$$

This system has non zero solutions $A_{k}, k=0,1,2,3$, if and only if

$$
\left|\begin{array}{cccc}
-\left(1+l+l^{2}\right) & -(2 s+1) & 0 & 0 \\
3 & 3-l(l+1) & \frac{2}{(2 s-1) !} & 0 \\
0 & 0 & 3-l(l+1) & 3 \\
0 & 0 & 2 s-1 & -1-l(l+1)
\end{array}\right|=0 .
$$


The last condition gives

$$
s= \pm \frac{l(l-1)(l+1)(l+2)}{6}, \quad l=2,3, \ldots
$$

which are precisely the frequencies $\frac{14,16}{16}$ of the algebraically special perturbations of the Schwarzschild geometry!; with the + sign, in accord with the convention we follow here for the time dependence of the perturbing field in its Fourier decomposition (equation (2)).

When condition (216) is satisfied the non zero solutions $A_{k}, k=0,1,2,3$, of the system of equations (211) - (214) is given by

$$
A_{1}=-\frac{l^{2}+l+1}{2 s+1} A_{0}, \quad A_{2}=-\frac{3(2 s) !}{2 s+1} A_{0}, \quad A_{3}=-\frac{\left(l^{2}+l-3\right)(2 s) !}{2 s+1} A_{0},
$$

where $A_{0}$ is arbitrary and where it is understood that $s=\frac{l(l-1)(l+1)(l+2)}{6}$. This completes the proof.

C. $\chi \int \frac{\mathrm{d} r_{*}}{\chi^{2}}$ in terms of truncated confluent hypergeometric functions of the first kind

Theorem 3 very strongly suggests that the polynomial solution $\mathrm{P}(u)$ of degree $2 s+1$ we found in subsection VC to equation (179), given by equations (181) and (182), does admit expansion of the form (201), in terms of truncated confluent hypergeometric functions of the first kind. This in turn implies that the polynomial $\mathrm{P}(w)$ which appears in the second Liouvillian solution $\chi \int \frac{\mathrm{d} r_{*}}{\chi^{2}}$, given by the family G7, can be expressed as a sum of truncated confluent hypergeometric functions of the first kind. It turns out that this is indeed the case, and it is the content of the following Theorem.

Theorem 4 The second Liouvillian solution $\chi \int \frac{\mathrm{d} r_{*}}{\chi^{2}}$ to the master equation (6), given by the family G7, in the case of the gravitational perturbations of the Schwarzschild geometry, is a product of elementary functions, one of them being a polynomial $\mathrm{P}(w)$ which admits an expansion in terms of truncated confluent hypergeometric functions of the first kind with appropriate function coefficients. In particular the following holds

$$
\begin{aligned}
\chi \int \frac{\mathrm{d} r_{*}}{\chi^{2}} & =\frac{\mathrm{P}(w) e^{\frac{s}{2} r}}{r(r-2)^{s}}= \\
& =\frac{e^{\frac{s}{2} r}}{r(r-2)^{s}}\left(\sum_{k=0}^{1} A_{k} \phi((2 s+1)-k ;-s w)+\sum_{k=2}^{3} A_{k} F(k-(2 s+1), 1-2 s ;-s w)\right),
\end{aligned}
$$


where $w=r-2$,

$$
\begin{gathered}
\phi(2 s+1 ;-s w)=(-s w)^{2 s} F(-1,2 s+1 ;-s w)=(s w)^{2 s}\left(1+\frac{s}{2 s+1} w\right), \\
\phi(2 s ;-s w)=(-s w)^{2 s} F(0,2 s+1 ;-s w)=(s w)^{2 s}, \\
F(-(2 s-1), 1-2 s ;-s w)=1-s w+\frac{(s w)^{2}}{2 !}-\frac{(s w)^{3}}{3 !}+\ldots-\frac{(s w)^{2 s-1}}{(2 s-1) !}, \\
F(-(2 s-2), 1-2 s ;-s w)=1-\frac{2-2 s}{1-2 s} s w+\frac{3-2 s}{1-2 s} \frac{(s w)^{2}}{2 !}+\ldots+\frac{(s w)^{2 s-2}}{(2 s-1) !} \\
A_{1}=-\frac{l^{2}+l+1}{2 s+1} A_{0}, A_{2}=-\frac{3(2 s) !}{2 s+1} A_{0}, A_{3}=-\frac{\left(l^{2}+l-3\right)(2 s) !}{2 s+1} A_{0}, A_{0}=\frac{s^{-2-2 s}(1+2 s)}{(l-1)(l+2)},
\end{gathered}
$$

where the coefficients $\mathrm{P}_{\mathrm{n}}$ of the polynomial $\mathrm{P}(w)=\sum_{\mathrm{n}=0}^{1+2 s} \mathrm{P}_{\mathrm{n}} w^{\mathrm{n}}$, of degree $2 s+1$, are given by equations (109), (110), and (117), and where $s=\frac{l(l-1)(l+1)(l+2)}{6}, l=2,3, \ldots$.

\section{Proof}

We can easily verify that if we choose

$$
A_{0}=\frac{s^{-2-2 s}(1+2 s)}{(l-1)(l+2)}
$$

then the expansion (equation (201))

$$
\mathrm{P}_{2 s+1}(u)=\sum_{k=0}^{1} A_{k} \phi((2 s+1)-k ; u)+\sum_{k=2}^{3} A_{k} F(k-(2 s+1), 1-2 s ; u)
$$

indeed holds, where $A_{1}, A_{2}, A_{3}$ are given by equation (218), and

$$
\mathrm{P}_{2 s+1}(u)=\sum_{\mathrm{n}=0}^{2 s+1} P_{\mathrm{n}} u^{\mathrm{n}}
$$

where the coefficients $P_{\mathrm{n}}$ are given by equations (181) and (182), and where it is understood that $s=\frac{l(l-1)(l+1)(l+2)}{6}$.

$\mathrm{P}_{2 s+1}(u)$ is a polynomial solution to equation (179). There is an associated polynomial solution $\mathrm{P}(w)=\sum_{\mathrm{n}=0}^{1+2 s} \mathrm{P}_{\mathrm{n}} w^{\mathrm{n}}$, of degree $2 s+1$, to equation (178), with coefficients $\mathrm{P}_{\mathrm{n}}$ given by equations (109), (110), and (117). From $u=-s w$ and $P_{\mathrm{n}}=\frac{\mathrm{P}_{\mathrm{n}}}{(-s)^{\mathrm{n}}}, 0 \leq \mathrm{n} \leq 2 s+1$, (equations (180) and (183) correspondingly), we conclude that $\mathrm{P}_{2 s+1}(u)$ and $\mathrm{P}(w)$ are equal when they are evaluated at $u=-s w$ and $w$ respectively. Indeed,

$$
\mathrm{P}_{2 s+1}(u)=\sum_{\mathrm{n}=0}^{2 s+1} P_{\mathrm{n}} u^{\mathrm{n}}=\sum_{\mathrm{n}=0}^{2 s+1} \frac{\mathrm{P}_{\mathrm{n}}}{(-s)^{\mathrm{n}}}(-s)^{\mathrm{n}} w^{\mathrm{n}}=\sum_{\mathrm{n}=0}^{2 s+1} \mathrm{P}_{\mathrm{n}} w^{\mathrm{n}}=\mathrm{P}(w) .
$$


Equations (201) and (220) yield

$$
\begin{aligned}
\mathrm{P}(w) & =\sum_{\mathrm{n}=0}^{2 s+1} \mathrm{P}_{\mathrm{n}} w^{\mathrm{n}}= \\
& =\sum_{k=0}^{1} A_{k} \phi((2 s+1)-k ;-s w)+\sum_{k=2}^{3} A_{k} F(k-(2 s+1), 1-2 s ;-s w) .
\end{aligned}
$$

$\mathrm{P}(w)$ is precisely the polynomial which appears in the second Liouvillian solution $\chi \int \frac{\mathrm{d} r_{*}}{\chi^{2}}$, initially found by Chandrasekhar $\stackrel{14}{ }$, to the RWE (RWE is the master equation (6) when $\beta=-3$ ), which describes the first order gravitational perturbations of the Schwarzschild geometry. Equations (93), (118) and (221) give

$$
\begin{aligned}
\chi \int \frac{\mathrm{d} r_{*}}{\chi^{2}} & =\frac{\mathrm{P}(w) e^{\frac{s}{2} r}}{r(r-2)^{s}}= \\
& =\frac{e^{\frac{s}{2} r}}{r(r-2)^{s}}\left(\sum_{k=0}^{1} A_{k} \phi((2 s+1)-k ;-s w)+\sum_{k=2}^{3} A_{k} F(k-(2 s+1), 1-2 s ;-s w)\right),
\end{aligned}
$$

where $w=r-2$. From equations (272), (190), and (191), we have

$$
\begin{aligned}
\phi(2 s+1 ;-s w) & =(-s w)^{2 s} F(-1,2 s+1 ;-s w)=(-s w)^{2 s}\left(1-\frac{1}{2 s+1}(-s w)\right)= \\
& =(s w)^{2 s}\left(1+\frac{s}{2 s+1} w\right), \\
\phi(2 s ;-s w) & =(-s w)^{2 s} F(0,2 s+1 ;-s w)=(s w)^{2 s}, \\
F(-(2 s-1), 1-2 s ;-s w) & =1+(-s w)+\frac{(-s w)^{2}}{2 !}+\frac{(-s w)^{3}}{3 !}+\ldots+\frac{(-s w)^{2 s-1}}{(2 s-1) !}= \\
& =1-s w+\frac{(s w)^{2}}{2 !}-\frac{(s w)^{3}}{3 !}+\ldots-\frac{(s w)^{2 s-1}}{(2 s-1) !}, \\
F(-(2 s-2), 1-2 s ;-s w) & =1+\frac{2-2 s}{1-2 s}(-s w)+\frac{3-2 s}{1-2 s} \frac{(-s w)^{2}}{2 !}+\ldots+\frac{(-1)}{1-2 s} \frac{(-s w)^{2 s-2}}{(2 s-2) !}= \\
& =1-\frac{2-2 s}{1-2 s} s w+\frac{3-2 s}{1-2 s} \frac{(s w)^{2}}{2 !}+\ldots+\frac{(s w)^{2 s-2}}{(2 s-1) !},
\end{aligned}
$$

and where, $A_{0}$, and $A_{1}, A_{2}, A_{3}$ are given respectively by equations (218) and (218). Moreover, it is understood that $s=\frac{l(l-1)(l+1)(l+2)}{6}, l=2,3, \ldots$. This completes the proof.

In subsections $\mathrm{VC}$ and $\mathrm{VD}$, by using Kovacic's algorithm, we showed that the second Liouvillian solution $\chi \int \frac{\mathrm{d} r_{*}}{\chi^{2}}$ has an elementary functions answer for every $l=2,3, \ldots$. In this subsection we go one step further and combine the results, derived in subsections $\mathrm{VC}$ and VD, from applying Kovacic's algorithm to the RWE, with the extension of Hautot's results 
we obtained in subsection VIIB, and conclude, in equation (222), that the polynomial $\mathrm{P}(w)$ appearing in the elementary functions answer of $\chi \int \frac{\mathrm{d} r_{*}}{\chi^{2}}$, is in fact a linear combination of truncated confluent hypergeometric functions of the first kind, with appropriate function coefficients.

\section{The simplest case $l=2$}

To give an example we consider the case where $l$ takes its lowest value $l=2$. Equation (216), with the $+\operatorname{sign}$, gives $s=4$ when $l=2$, and, as a result, equation (222) gives

$$
\begin{aligned}
\chi \int \frac{\mathrm{d} r_{*}}{\chi^{2}} & =\frac{\mathrm{P}(w) e^{2 r}}{r(r-2)^{4}}= \\
& =\frac{e^{2 r}}{r(r-2)^{4}}\left(A_{0} \phi(9 ;-4 w)+A_{1} \phi(8 ;-4 w)+A_{2} F(-7,-7 ;-4 w)+A_{3} F(-6,-7 ;-4 w)\right),
\end{aligned}
$$

where $w=r-2$.

The coefficients $\mathrm{P}_{\mathrm{n}}$ of the polynomial $\mathrm{P}(w)=\sum_{\mathrm{n}=0}^{1+2 s} \mathrm{P}_{\mathrm{n}} w^{\mathrm{n}}$, appearing in the elementary functions answer (227) of $\chi \int \frac{\mathrm{d} r_{*}}{\chi^{2}}$, are given by equations (109), (110), and (117), and so we obtain

$\mathrm{P}_{9}(w)=-\frac{945}{16384}+\frac{1755}{8192} w-\frac{405}{1024} w^{2}+\frac{495}{1024} w^{3}-\frac{225}{512} w^{4}+\frac{81}{256} w^{5}-\frac{3}{16} w^{6}+\frac{3}{32} w^{7}+\frac{1}{32} w^{8}+\frac{1}{16} w^{9}$,

where the subscript 9 in $\mathrm{P}_{9}(w)$ indicates the degree of the polynomial $\mathrm{P}_{9}(w)$. When $l=2$

the coefficients $A_{0}$, and $A_{1}, A_{2}, A_{3}$, given respectively by equations (218) and (218), are equal to

$$
A_{0}=\frac{9}{4194304}, A_{1}=-\frac{7}{4194304}, A_{2}=-\frac{945}{32768}, A_{3}=-\frac{945}{32768}
$$

When $s=4$ the polynomials $\phi(2 s+1 ;-s w), \phi(2 s ;-s w), F(-(2 s-1), 1-2 s ;-s w)$, and $F(-(2 s-2), 1-2 s ;-s w)$, given respectively by equations (223), (224), (225), and (226), 
are equal to

$$
\begin{aligned}
\phi(2 s+1 ;-s w) & =\phi(9 ;-4 w)=(4 w)^{8}\left(1+\frac{4}{9} w\right), \\
\phi(2 s ;-s w) & =\phi(8 ;-4 w)=(4 w)^{8} \\
F(-(2 s-1), 1-2 s ;-s w) & =F(-7,-7 ;-4 w)= \\
& =1-4 w+\frac{(4 w)^{2}}{2 !}-\frac{(4 w)^{3}}{3 !}+\frac{(4 w)^{4}}{4 !}-\frac{(4 w)^{5}}{5 !}+\frac{(4 w)^{6}}{6 !}-\frac{(4 w)^{7}}{7 !}, \\
F(-(2 s-2), 1-2 s ;-s w) & =F(-6,-7 ;-4 w)= \\
& =1-\frac{6}{7} 4 w+\frac{5}{7} \frac{(4 w)^{2}}{2 !}-\frac{4}{7} \frac{(4 w)^{3}}{3 !}+\frac{3}{7} \frac{(4 w)^{4}}{4 !}-\frac{2}{7} \frac{(4 w)^{5}}{5 !}+\frac{1}{7} \frac{(4 w)^{6}}{6 !},
\end{aligned}
$$

We easily check that (221) is indeed valid when $s=4$, i.e., we easily check that

$$
\mathrm{P}_{9}(w)=A_{0} \phi(9 ;-4 w)+A_{1} \phi(8 ;-4 w)+A_{2} F(-7,-7 ;-4 w)+A_{3} F(-6,-7 ;-4 w),
$$

where $\mathrm{P}_{9}(w)$ is given by equation (228), $A_{0}, A_{1}, A_{2}, A_{3}$ are given by equation (229), and $\phi(9 ;-4 w), \phi(8 ;-4 w), F(-7,-7 ;-4 w)$, and $F(-6,-7 ;-4 w)$, are given respectively by equations (230), (231), (232), and (233).

Thus, by combining the results of subsections $\mathrm{VC}$, $\mathrm{VD}$ and $\mathrm{VIIB}$, with the results of this subsection, we conclude that when $l=2$ the second Liouvillian solution is

$$
\begin{aligned}
\chi \int \frac{\mathrm{d} r_{*}}{\chi^{2}} & =\frac{e^{2 r}}{r(r-2)^{4}}\left(\frac{9}{4194304}(4 w)^{8}\left(1+\frac{4}{9} w\right)-\frac{7}{4194304}(4 w)^{8}-\right. \\
& -\frac{945}{32768}\left(1-4 w+\frac{(4 w)^{2}}{2 !}-\frac{(4 w)^{3}}{3 !}+\frac{(4 w)^{4}}{4 !}-\frac{(4 w)^{5}}{5 !}+\frac{(4 w)^{6}}{6 !}-\frac{(4 w)^{7}}{7 !}\right)- \\
& \left.-\frac{945}{32768}\left(1-\frac{6}{7} 4 w+\frac{5}{7} \frac{(4 w)^{2}}{2 !}-\frac{4}{7} \frac{(4 w)^{3}}{3 !}+\frac{3}{7} \frac{(4 w)^{4}}{4 !}-\frac{2}{7} \frac{(4 w)^{5}}{5 !}+\frac{1}{7} \frac{(4 w)^{6}}{6 !}\right)\right)
\end{aligned}
$$

where $w=r-2$. 


\section{D. $\chi \int \frac{\mathrm{d} r_{*}}{\chi^{2}}$ in terms of associated Laguerre polynomials}

As we noted in subsection VID 2, associated Laguerre polynomials $L_{\mathrm{n}}^{(a)}(u)$ and confluent hypergeometric functions of the first kind $F(\mathrm{n}, a+1, u)$ are related via

$$
L_{\mathrm{n}}^{(a)}(u)=\left(\begin{array}{c}
\mathrm{n}+a \\
\mathrm{n}
\end{array}\right) F(\mathrm{n}, a+1, u)
$$

where

$$
\left(\begin{array}{l}
A \\
K
\end{array}\right)=\frac{A(A-1)(A-2) \cdots(A-K+1)}{K !}
$$

is the generalized binomial coefficient, i.e., $A \in R$ and $K \in N, R$ and $N$ are the set of real numbers and the set of natural numbers respectively.

Thus the associated Laguerre polynomials $L_{\mathrm{n}}^{(a)}(u)$ satisfy the differential equation

$$
u L_{\mathrm{n}}^{(a)^{\prime \prime}}(u)+(a+1-u) L_{\mathrm{n}}^{(a)^{\prime}}(u)+\mathrm{n} L_{\mathrm{n}}^{(a)}(u)=0 .
$$

Equation (236) is in fact equivalent to equation (170), given in subsection VID 2, which expresses the relation between associated Laguerre polynomials and confluent hypergeometric functions of the first kind.

Relation (236) suggests that the analysis of the previous subsection can be repeated by expanding $\mathrm{P}(w)$ not as a sum of confluent hypergeometric functions of the first kind, with appropriate function coefficients, as it is done in equation (221), but instead, by expanding $\mathrm{P}(w)$ as a sum of associated Laguerre polynomialls, with appropriate function coefficients, in the form

$$
\begin{aligned}
\mathrm{P}(w) & =\sum_{\mathrm{n}=0}^{2 s+1} \mathrm{P}_{\mathrm{n}} w^{\mathrm{n}}= \\
& =\sum_{k=0}^{1} \mathcal{A}_{k} \varphi((2 s+1)-k ;-s w)+\sum_{k=2}^{3} \mathcal{A}_{k} L_{(2 s+1)-k}^{(-2 s)}(-s w),
\end{aligned}
$$

where, from equation (236) we have

$$
\begin{aligned}
L_{1}^{(2 s)}(-s w) & =(2 s+1) F(-1,2 s+1 ;-s w), \\
L_{0}^{(2 s)}(-s w) & =F(0,2 s+1 ;-s w), \\
L_{2 s-1}^{(-2 s)}(-s w) & =-F(-(2 s-1), 1-2 s ;-s w), \\
L_{2 s-2}^{(-2 s)}(-s w) & =(2 s-1) F(-(2 s-2), 1-2 s ;-s w),
\end{aligned}
$$


and subsequently, by taking also into account equations (240), (241), (242), and (243), we have

$$
\begin{aligned}
\varphi(2 s+1 ;-s w) & =(-s w)^{2 s} L_{1}^{(2 s)}(-s w)=(s w)^{2 s}(s w+2 s+1), \\
\varphi(2 s ;-s w) & =(-s w)^{2 s} L_{0}^{(2 s)}(-s w)=(s w)^{2 s}, \\
L_{2 s-1}^{(-2 s)}(-s w) & =-(F(-(2 s-1), 1-2 s ;-s w))= \\
& =-\left(1-s w+\frac{(s w)^{2}}{2 !}-\frac{(s w)^{3}}{3 !}+\ldots-\frac{(s w)^{2 s-1}}{(2 s-1) !}\right), \\
L_{2 s-2}^{(-2 s)}(-s w) & =(2 s-1) F(-(2 s-2), 1-2 s ;-s w)= \\
& =(2 s-1)\left(1-\frac{2-2 s}{1-2 s} s w+\frac{3-2 s}{1-2 s} \frac{(s w)^{2}}{2 !}+\ldots+\frac{(s w)^{2 s-2}}{(2 s-1) !}\right) .
\end{aligned}
$$

$\mathrm{P}(w)$ is the polynomial solution of degree $2 s+1$, to equation (178), with coefficients $\mathrm{P}_{\mathrm{n}}$ given by equations (109), (110), and (117).

We follow through the analysis carried out in subsections VIIB and VIIC: By taking into account equation (220), by substituting the ansatz (239) into equation (146) and by using appropriate recurrence relations for the four polynomial solutions $\varphi(2 s+1 ;-s w)$, $\varphi(2 s ;-s w), L_{2 s-1}^{(-2 s)}(-s w)$, and $L_{2 s-2}^{(-2 s)}(-s w)$, we obtain a $4 \times 4$ homogeneous system for the coefficients $\mathcal{A}_{k}, k=0,1,2,3$.

It is straightforward to verify that the necessary and sufficient condition for obtaining non zero solutions to this system is $s=\frac{l(l-1)(l+1)(l+2)}{6}, l=2,3, \ldots$. When this condition is satisfied we obtain

$$
\mathcal{A}_{1}=-\left(l^{2}+l+1\right) \mathcal{A}_{0}, \quad \mathcal{A}_{2}=3(2 s) ! \mathcal{A}_{0}, \quad \mathcal{A}_{3}=-\frac{\left(l^{2}+l-3\right)(2 s) !}{2 s-1} \mathcal{A}_{0},
$$

We easily find that if we choose

$$
\mathcal{A}_{0}=\frac{s^{-2-2 s}}{(l-1)(l+2)}
$$

then the expansion (equation (239))

$$
\mathrm{P}(w)=\sum_{k=0}^{1} \mathcal{A}_{k} \varphi((2 s+1)-k ;-s w)+\sum_{k=2}^{3} \mathcal{A}_{k} L_{(2 s+1)-k}^{(-2 s)}(-s w),
$$

indeed holds, where $\mathcal{A}_{1}, \mathcal{A}_{2}, \mathcal{A}_{3}$ are given by equation (248), and

$$
\mathrm{P}(w)=\sum_{\mathrm{n}=0}^{2 s+1} \mathrm{P}_{\mathrm{n}} w^{\mathrm{n}}
$$


where the coefficients $\mathrm{P}_{\mathrm{n}}$ are given by equations (109), (110), and (117), and where it is understood that $s=\frac{l(l-1)(l+1)(l+2)}{6}$.

$\mathrm{P}(w)$ is the polynomial which appears in the second Liouvillian solution $\chi \int \frac{\mathrm{d} r_{*}}{\chi^{2}}$, initially found by Chandrasekhar ${ }^{14}$, to the RWE. Equations (93), (118) and (239) give

$$
\begin{aligned}
\chi \int \frac{\mathrm{d} r_{*}}{\chi^{2}} & =\frac{\mathrm{P}(w) e^{\frac{s}{2} r}}{r(r-2)^{s}}= \\
& =\frac{e^{\frac{s}{2} r}}{r(r-2)^{s}}\left(\sum_{k=0}^{1} \mathcal{A}_{k} \varphi((2 s+1)-k ;-s w)+\sum_{k=2}^{3} \mathcal{A}_{k} L_{(2 s+1)-k}^{(-2 s)}(-s w)\right),
\end{aligned}
$$

where $w=r-2$. The four polynomials $\varphi(2 s+1 ;-s w), \varphi(2 s ;-s w), L_{2 s-1}^{(-2 s)}(-s w)$, and $L_{2 s-2}^{(-2 s)}(-s w)$, are given respectively by equations (244), (245), (246), and (247).

We conclude, in equation (251), that the polynomial $\mathrm{P}(w)$ appearing in the elementary functions answer of $\chi \int \frac{\mathrm{d} r_{*}}{\chi^{2}}$, admits an expansion in terms of associated Laguerre polynomials, with appropriate function coefficients. We thus have the following Theorem.

Theorem 5 The second Liouvillian solution $\chi \int \frac{\mathrm{d} r_{*}}{\chi^{2}}$ to the master equation (6), given by the family G\%, in the case of the gravitational perturbations of the Schwarzschild geometry, is a product of elementary functions, one of them being a polynomial $\mathrm{P}(w)$ which admits an expansion in terms of associated Laguerre polynomials with appropriate function coefficients. In particular the following holds

$$
\begin{aligned}
\chi \int \frac{\mathrm{d} r_{*}}{\chi^{2}} & =\frac{\mathrm{P}(w) e^{\frac{s}{2} r}}{r(r-2)^{s}}= \\
& =\frac{e^{\frac{s}{2} r}}{r(r-2)^{s}}\left(\sum_{k=0}^{1} \mathcal{A}_{k} \varphi((2 s+1)-k ;-s w)+\sum_{k=2}^{3} \mathcal{A}_{k} L_{(2 s+1)-k}^{(-2 s)}(-s w)\right),
\end{aligned}
$$

where $w=r-2$,

$$
\begin{gathered}
\varphi(2 s+1 ;-s w)=(-s w)^{2 s} L_{1}^{(2 s)}(-s w)=(s w)^{2 s}(s w+2 s+1), \\
\varphi(2 s ;-s w)=(-s w)^{2 s} L_{0}^{(2 s)}(-s w)=(s w)^{2 s}, \\
L_{2 s-1}^{(-2 s)}(-s w)=-\left(1-s w+\frac{(s w)^{2}}{2 !}-\frac{(s w)^{3}}{3 !}+\ldots-\frac{(s w)^{2 s-1}}{(2 s-1) !}\right), \\
L_{2 s-2}^{(-2 s)}(-s w)=(2 s-1)\left(1-\frac{2-2 s}{1-2 s} s w+\frac{3-2 s}{1-2 s} \frac{(s w)^{2}}{2 !}+\ldots+\frac{(s w)^{2 s-2}}{(2 s-1) !}\right), \\
\mathcal{A}_{1}=-\left(l^{2}+l+1\right) \mathcal{A}_{0}, \mathcal{A}_{2}=3(2 s) ! \mathcal{A}_{0}, \mathcal{A}_{3}=-\frac{\left(l^{2}+l-3\right)(2 s) !}{2 s-1} \mathcal{A}_{0}, \mathcal{A}_{0}=\frac{s^{-2-2 s}}{(l-1)(l+2)},
\end{gathered}
$$


where the coefficients $\mathrm{P}_{\mathrm{n}}$ of the polynomial $\mathrm{P}(w)=\sum_{\mathrm{n}=0}^{1+2 s} \mathrm{P}_{\mathrm{n}} w^{\mathrm{n}}$, of degree $2 s+1$, are given by equations (109), (110), and (117), and where $s=\frac{l(l-1)(l+1)(l+2)}{6}, l=2,3, \ldots$.

In subsection $\mathrm{VIIC}$ we also proved that $\mathrm{P}(w)$ admits also an expansion in terms of truncated confluent hypergeometric functions of the first kind with appropriate function coefficients.

\section{The simplest case $l=2$}

To give an example we consider the case where $l$ takes its lowest value $l=2$. Equation (216), gives $s=4$ when $l=2$, and, as a result, equation (251) gives

$$
\begin{aligned}
\chi \int \frac{\mathrm{d} r_{*}}{\chi^{2}} & =\frac{\mathrm{P}_{9}(w) e^{2 r}}{r(r-2)^{4}}= \\
& =\frac{e^{2 r}}{r(r-2)^{4}}\left(\mathcal{A}_{0} \varphi(9 ;-4 w)+\mathcal{A}_{1} \varphi(8 ;-4 w)+\mathcal{A}_{2} L_{7}^{(-8)}(-4 w)+\mathcal{A}_{3} L_{6}^{(-8)}(-4 w)\right),
\end{aligned}
$$

where $w=r-2$. The polynomial $\mathrm{P}_{9}(w)$ of degree 9 is given by equation (228).

We evaluate the coefficients $\mathcal{A}_{0}, \mathcal{A}_{1}, \mathcal{A}_{2}, \mathcal{A}_{3}$ from equations (248) and (249), and we evaluate the polynomials $\varphi(9 ;-4 w), \varphi(8 ;-4 w), L_{7}^{(-8)}(-4 w)$, and $L_{6}^{(-8)}(-4 w)$ from equations (244), (245), (246), and (247) respectively, and we obtain

$$
\begin{aligned}
\chi \int \frac{\mathrm{d} r_{*}}{\chi^{2}} & =\frac{e^{2 r}}{r(r-2)^{4}}\left(\frac{1}{4194304}(4 w)^{8}(4 w+9)-\frac{7}{4194304}(4 w)^{8}-\right. \\
& -\frac{945}{32768}\left(1-4 w+\frac{(4 w)^{2}}{2 !}-\frac{(4 w)^{3}}{3 !}+\frac{(4 w)^{4}}{4 !}-\frac{(4 w)^{5}}{5 !}+\frac{(4 w)^{6}}{6 !}-\frac{(4 w)^{7}}{7 !}\right)- \\
& \left.-\frac{945}{229376}\left(7-6(4 w)+5 \frac{(4 w)^{2}}{2 !}-4 \frac{(4 w)^{3}}{3 !}+3 \frac{(4 w)^{4}}{4 !}-2 \frac{(4 w)^{5}}{5 !}+\frac{(4 w)^{6}}{6 !}\right)\right)
\end{aligned}
$$

where $w=r-2$.

\section{THE REMAINING FAMILIES G3, E3, E7 AND S3}

We examine now the remaining families G3, E3, E7 and S3. In subsection VIIIA we show that the ODEs associated with all the remaining families G3, E3, E7 and S3 fall into 
the confluent Heun class after a trivial change of the independent variable $r$. In subsection VIIIB we use the elementary symmetries of the confluent Heun equation to show that the families G7 and G3 are related with such an elementary symmetry, and also to show that the families E7 and E3 are related with such an elementary symmetry.

In subsection VIIIC we prove that the family S3 does not give rise to a Liouvillian solution to the master equation (6) . In subsection VIIID we give strong evidence which supports the thesis that the families G3, E7 and E3 do not give rise to Liouvillian solutions to the master equation (6). In subsection VIIIE we conclude that the four remaining families G3, E3, E7 and S3 are reduced into the two families G3 and E7 and we put forward the conjecture, by taking into account the evidence given in subsection VIIID, that the families G3 and E7 do not give rise to Liouvillian solutions to the master equation (6).

Thus in this section we consider problem 3 in the list of most difficult problems, mentioned in subsection IA, we have to solve when we apply Kovacic's algorithm. In fact problem 3 in subsection $\llbracket \mathrm{A}$, in the problem under consideration, amounts to proving that the families G3, E7, E3, and S3 do not give rise to Liouvillian solutions to the master equation (66).

Couch and Holder ${ }^{26}$ address also problem 3 . We improve the results derived by C.H. ${ }^{26}$ on the solution of problem 3. Namely, the results derived in subsections VIIIA, VIIIB, VIIIC, VIIID, and VIIIE, are new. There are some discrepancies between the results we derive on the solution of problem 3 and the results derived by C.H. ${ }^{26}$ on the solution of problem 3 . We compare our results on the solution of problem 3 with the results of C.H. on the solution of

problem 3 in subsection VIIIF. In the last subsection VIIIG we make some comments on the proper implementation of Kovacic's algorithm for any second order ODE with rational function coefficients.

\section{A. The remaining families fall into the confluent Heun class}

The first key observation is that the ODEs

$$
\mathrm{P}^{\prime \prime}+2 \theta \mathrm{P}^{\prime}+\left(\theta^{2}+\theta^{\prime}-\nu\right) \mathrm{P}=0
$$

(equation (73) ), where

$$
\nu(r)=\frac{\frac{s^{2}}{4} r^{4}+l(l+1) r^{2}+2[\beta-l(l+1)-1] r+3-4 \beta}{r^{2}(r-2)^{2}}
$$


(equation (65)), and $\beta=-3,0,1$ distinguishes the various types of external perturbations, gravitational, electromagnetic or massless scalar fields respectively, associated with the families G3, E3, E7 and S3 fall into the confluent Heun class after a trivial change of the independent variable. It is to be noted that the ODE associated with the family G7, which

gives rise to the Liouvillian solution $\chi \int \frac{\mathrm{d} r_{*}}{\chi^{2}}$, falls also into the confluent Heun class after the same trivial change of the independent variable. We also include the family G7 because it is going to be useful in subsection $\mathrm{VD}$.

\section{The family G7}

In the case of the family G7 we have (equation (66))

$$
\theta(r)=\frac{-3 / 2}{r}+\frac{\frac{1}{2}-s}{r-2}+\frac{s}{2}
$$

and therefore equation (173) reads

$$
r(r-2) \mathrm{P}^{\prime \prime}(r)+\left(6-2 r(2 s+1)+r^{2} s\right) \mathrm{P}^{\prime}(r)+(2-l(l+1)+6 s-r s(2 s+1)) \mathrm{P}(r)=0 .
$$

Equation (254) after the change of the independent variable $r=2 z$ becomes

$$
z(z-1) \mathrm{p}^{\prime \prime}(z)+\left(3-2 z(2 s+1)+2 z^{2} s\right) \mathrm{p}^{\prime}(z)+(2-l(l+1)+6 s-2 z s(2 s+1)) \mathrm{p}(z)=0
$$

which falls into the confluent Heun class. It is trivially verified that equation (254) admits a polynomial solution $\mathrm{P}(r)$ if and only if equation (255) admits a polynomial solution $\mathrm{p}(z)$. If such polynomial solutions exist then $\mathrm{p}(z)=\mathrm{P}(2 z)$.

We have in turn now for the remaining families G3, E3, E7 and S3:

\section{The family G3}

In the case of the family G3 we have (equation (65))

$$
\theta(r)=\frac{5 / 2}{r}+\frac{\frac{1}{2}-s}{r-2}+\frac{s}{2}
$$

and therefore equation (73) reads

$$
r(r-2) \mathrm{P}^{\prime \prime}(r)+\left(-10-2 r(2 s-3)+r^{2} s\right) \mathrm{P}^{\prime}(r)+(6-l(l+1)-10 s-r s(2 s-3)) \mathrm{P}(r)=0 .
$$

Equation (256) after the change of the independent variable $r=2 z$ becomes

$$
z(z-1) \mathrm{p}^{\prime \prime}(z)+\left(-5-2 z(2 s-3)+2 z^{2} s\right) \mathrm{p}^{\prime}(z)+(6-l(l+1)-10 s-2 z s(2 s-3)) \mathrm{p}(z)=0
$$


which falls into the confluent Heun class. It is trivially verified that equation (256) admits a polynomial solution $\mathrm{P}(r)$ if and only if equation (257) admits a polynomial solution $\mathrm{p}(z)$. If such polynomial solutions exist then $\mathrm{p}(z)=\mathrm{P}(2 z)$.

\section{The family E7}

In the case of the family E7 we have (equation (70) )

$$
\theta(r)=\frac{-1 / 2}{r}+\frac{\frac{1}{2}-s}{r-2}+\frac{s}{2},
$$

and therefore equation (73) reads

$$
r(r-2) \mathrm{P}^{\prime \prime}(r)+\left(2-4 r s+r^{2} s\right) \mathrm{P}^{\prime}(r)+\left(-l(l+1)+2 s-2 s^{2} r\right) \mathrm{P}(r)=0 .
$$

Equation (258) after the change of the independent variable $r=2 z$ becomes

$$
z(z-1) \mathrm{P}^{\prime \prime}(z)+\left(1-4 z s+2 z^{2} s\right) \mathrm{P}^{\prime}(z)+\left(-l(l+1)+2 s-4 s^{2} z\right) \mathrm{P}(z)=0
$$

which falls into the confluent Heun class. It is trivially verified that equation (258) admits a polynomial solution $\mathrm{P}(r)$ if and only if equation (259) admits a polynomial solution $\mathrm{p}(z)$. If such polynomial solutions exist then $\mathrm{p}(z)=\mathrm{P}(2 z)$.

\section{The family E3}

In the case of the family E3 we have (equation (69))

$$
\theta(r)=\frac{3 / 2}{r}+\frac{\frac{1}{2}-s}{r-2}+\frac{s}{2}
$$

and therefore equation (73) reads

$$
r(r-2) \mathrm{P}^{\prime \prime}(r)+\left(-6-2 r(2 s-2)+r^{2} s\right) \mathrm{P}^{\prime}(r)+(-l(l+1)-6 s+2-2 r s(2 s-2)) \mathrm{P}(r)=0 .
$$

Equation (260) after the change of the independent variable $r=2 z$ becomes

$$
z(z-1) \mathrm{P}^{\prime \prime}(z)+\left(-3-2 z(2 s-2)+2 z^{2} s\right) \mathrm{P}^{\prime}(z)+(-l(l+1)-6 s+2-4 z s(2 s-2)) \mathrm{P}(z)=0
$$

which falls into the confluent Heun class. It is trivially verified that equation (260) admits a polynomial solution $\mathrm{P}(r)$ if and only if equation (261) admits a polynomial solution $\mathrm{p}(z)$. If such polynomial solutions exist then $\mathrm{p}(z)=\mathrm{P}(2 z)$.

\section{The family S3}

In the case of the family S3 we have (equation (68))

$$
\theta(r)=\frac{1 / 2}{r}+\frac{\frac{1}{2}-s}{r-2}+\frac{s}{2}
$$


and therefore equation (73) reads

$$
r(r-2) \mathrm{P}^{\prime \prime}(r)+\left(-2-2 r(2 s-1)+r^{2} s\right) \mathrm{P}^{\prime}(r)+(-l(l+1)-2 s-r s(2 s-1)) \mathrm{P}(r)=0 .
$$

Equation (262) after the change of the independent variable $r=2 z$ becomes

$$
z(z-1) \mathrm{P}^{\prime \prime}(z)+\left(-1-2 z(2 s-1)+2 z^{2} s\right) \mathrm{P}^{\prime}(z)+(-l(l+1)-2 s-2 z s(2 s-1)) \mathrm{P}(z)=0
$$

which falls into the confluent Heun class. It is trivially verified that equation (262) admits a polynomial solution $\mathrm{P}(r)$ if and only if equation (263) admits a polynomial solution $\mathrm{p}(z)$. If such polynomial solutions exist then $\mathrm{p}(z)=\mathrm{P}(2 z)$.

\section{B. Elementary symmetries of the confluent Heun equation and the remaining families}

It is well known (see e.g. $\underline{78}, \underline{80}$ ) that there are 16 variable substitutions which preserve the form of the confluent Heun equation

$$
z(z-1) \mathrm{p}^{\prime \prime}(z)+\left(a z^{2}+b z+c\right) \mathrm{p}^{\prime}(z)+(d+e z) \mathrm{p}(z)=0 .
$$

For convenience, we rewrite equation (264) in the form

$$
\mathcal{R}_{(a, b, c, d, e)} \mathrm{p}(z)=0
$$

where $\mathcal{R}_{(a, b, c, d, e)}$ is the linear operator

$$
\mathcal{R}_{(a, b, c, d, e)}=z(z-1) \frac{\mathrm{d}^{2}}{\mathrm{~d} z^{2}}++\left(a z^{2}+b z+c\right) \frac{\mathrm{d}}{\mathrm{d} z}++(d+e z) .
$$

The subscript $(a, b, c, d, e)$ in $\mathcal{R}_{(a, b, c, d, e)}$ emphasizes the dependence of $\mathcal{R}_{(a, b, c, d, e)}$ on the parameters $a, b, c, d$ and $e$.

Out of these 16 variable substitutions the one which is the most relevant here is the following: The substitution

$$
\mathrm{p}(z)=z^{1+c} \mathrm{p}_{1}(z)
$$

in equation (264) yields

$$
R_{(a, b+2+2 c,-2-c, d+(b+c)(c+1), e+a c+a)} \mathrm{p}_{1}(z)=0 .
$$


Therefore we have the equivalence

$$
\mathcal{R}_{(a, b, c, d, e)} \mathrm{p}(z)=0 \Leftrightarrow R_{(a, b+2+2 c,-2-c, d+(b+c)(c+1), e+a c+a)} \mathrm{p}_{1}(z)=0 .
$$

We note that in transformation (267) there is no change of the independent variable $z$. Consequently the positions of the singular points remain fixed under this transformation. For this reason, transformation (267), and more generally transformations which do not

involve change of the independent variable are called homotopic ${ }^{54}$ (from the greek words "homo" = "same" and "topos"= "place".)

We prove now that the confluent Heun equations associated with the families G7 and G3 are related with the equivalence relation (269). The same applies to the confluent Heun equations associated with the families E7 and E3. This has implications for the solution of the problem we are considering in this section; in particular it gives useful equivalent formulation of the problem we need to solve for the family G3, and it reduces the consideration of the families E7 and E3 into the consideration of the family E7 only. The details are as follows:

\section{The families G7 and G3}

The confluent Heun equation associated with the family G7 is (equation (255))

$$
z(z-1) \mathrm{p}^{\prime \prime}(z)+\left(3-2 z(2 s+1)+2 z^{2} s\right) \mathrm{p}^{\prime}(z)+(2-l(l+1)+6 s-2 z s(2 s+1)) \mathrm{p}(z)=0 .
$$

Comparison with the general form of the confluent Heun equation (264) gives

$$
a=2 s, b=-2(2 s+1), c=3, d=2-l(l+1)+6 s, \text { and } e=-2 s(2 s+1) .
$$

According to the equivalence relation (269), the substitution

$$
\mathrm{p}(z)=z^{4} \mathrm{p}_{1}(z)
$$

into equation (255) yields

$z(z-1) \mathrm{p}_{1}^{\prime \prime}(z)+\left(-5-2 z(2 s-3)+2 z^{2} s\right) \mathrm{p}_{1}^{\prime}(z)+(6-l(l+1)-10 s-2 z s(2 s-3)) \mathrm{p}_{1}(z)=0$,

which is nothing but the confluent Heun equation associated with the family G3 (equation (257)). 
In subsections $\mathrm{VC}$ and $\mathrm{VII}$ we proved that the confluent Heun equation associated with the family G7 (equation (255)) admits a polynomial solution

$$
\mathrm{P}(u)=\sum_{\mathrm{n}=0}^{1+2 s} P_{\mathrm{n}} u^{\mathrm{n}}=\sum_{\mathrm{n}=0}^{1+2 s} P_{\mathrm{n}}(2 s(1-z))^{\mathrm{n}}=\mathrm{p}(z)=\mathrm{p}_{0}+\mathrm{p}_{1} z+\mathrm{p}_{2} z^{2}+\mathrm{p}_{3} z^{3}+\ldots+\mathrm{p}_{1+2 s} z^{1+2 s}
$$

of degree $1+2 s$ with coefficients $P_{\mathrm{n}}$ given by (equations (181) and (182) respectively)

$$
\begin{aligned}
P_{\mathrm{n}} & =-\frac{6 s^{-2 s-1}(2 s) !((l-1)(l+2)-3 s)[(\mathrm{n}-2 s)(l-1)(l+2)-6 s]}{\mathrm{n} !((l-1)(l+2)+6 s) s(l-1)^{3}(l+2)^{3}}, \\
P_{2 s} & =\frac{2(l-1)(l+2)-6}{s^{2 s+1}(l-1)^{2}(l+2)^{2}}, \quad P_{2 s+1}=-\frac{1}{s^{2 s+2}(l-1)(l+2)},
\end{aligned}
$$

where $0 \leq \mathrm{n} \leq 2 s-1$ and $s=\frac{l(l-1)(l+1)(l+2)}{6}, \quad l=2,3, \ldots$.

The key fact here is that the coefficients $\mathrm{p}_{0}, \mathrm{p}_{1}, \mathrm{p}_{2}, \mathrm{p}_{3}$ of the polynomial solution (272) to the confluent Heun equation (255) associated with the family G7 are different from zero for every value of the angular harmonic index $l=2,3, \ldots$. In fact we can easily show by using equations (181) and (182) that

$$
\operatorname{sgn}\left(\mathrm{p}_{\mathrm{n}}\right)=(-1)^{\mathrm{n}+1}, \mathrm{n}=0,1,2, \ldots, 2 s+1, \quad \forall \mathrm{l}=2,3, \ldots
$$

So in particular for the first four coefficients we have

$$
\mathrm{p}_{0}<0, \mathrm{p}_{1}>0, \mathrm{p}_{2}<0, \mathrm{p}_{3}>0, \forall l=2,3, \ldots
$$

In subsection VIIID we give strong evidence that the family G3 does not give rise to a Liouvillian solution to the master equation (6). We can reformulate the problem of proving that the family G3 does not give rise to a Liouvillian solution to the master equation (6) by using the equivalence of families G7 and G3 which was shown to exist by $\operatorname{setting~} \mathrm{p}(z)$ equal to $z^{4} \mathrm{p}_{1}(z)$ into equation (255). In the following two Corollaries we give two such reformulations relating the families G7 and G3.

Corollary 1 In order to prove that the the family G3 does not give rise to a Liouvillian solution to the master equation (6) it suffices to prove that the confluent Heun equation (255) associated with the family G7 admits only one polynomial solution, namely the polynomial solution we found in subsections $\overline{V C}$ and VIIA, given by equations (181), (182), and (272). 
Proof Let us assume that the confluent Heun equation (255) associated with the family G7 admits only one polynomial solution, namely the polynomial solution

$$
\mathrm{p}(z)=\sum_{\mathrm{n}=0}^{1+2 s} \mathrm{p}_{\mathrm{n}} z^{\mathrm{n}}=\mathrm{p}_{0}+\mathrm{p}_{1} z+\mathrm{p}_{2} z^{2}+\mathrm{p}_{3} z^{3}+\ldots+\mathrm{p}_{1+2 s} z^{1+2 s},
$$

we found in subsections $\mathrm{VC}$ and VIIA , given by equations (181), (182), and (272). The coefficients $\mathrm{p}_{0}, \mathrm{p}_{1}, \mathrm{p}_{2}, \mathrm{p}_{3}$ of this polynomial solution are different from zero, in particular we have $\mathrm{p}_{0}<0, \mathrm{p}_{1}>0, \mathrm{p}_{2}<0, \mathrm{p}_{3}>0, \forall l=2,3, \ldots$, (equation (274)).

This implies that the confluent Heun equation (257) associated with the family G3 does not admit any polynomial solution, because if it did, according to equation (277), the confluent Heun equation (255) associated with the family G3 would admit polynomial solution whose smallest power would be 4 . But this cannot happen since, according to equation (277), the coefficients $\mathrm{p}_{0}, \mathrm{p}_{1}, \mathrm{p}_{2}, \mathrm{p}_{3}$ are different from zero and we have assumed that the only polynomial solution to the confluent Heun equation (255) associated with the family G7 is the one given by equation (275). We conclude that the confluent Heun equation (257) associated with the family G3 does not admit any polynomial solution and therefore the family G3 does not give rise to any Liouvillian solution to the master equation (66). This completes the proof.

We note that, strictly speaking, for the proof of Corollary 1 we do not need equations (273) and (274), we only need to know that at least one of the coefficients $\mathrm{p}_{0}, \mathrm{p}_{1}, \mathrm{p}_{2}, \mathrm{p}_{3}$ is different from zero. This remark suggests a second reformulation of the problem of proving that the family G3 does not give rise to a Liouvillian solution to the master equation (6). This is the content of the next Corollary.

Corollary 2 In order to prove that the the family G3 does not give rise to a Liouvillian solution to the master equation (6) it suffices to prove that if the confluent Heun equation (255) associated with the family G7 admits a polynomial solution

$$
\mathrm{p}(z)=\sum_{\mathrm{n}=0}^{1+2 s} \mathrm{p}_{\mathrm{n}} z^{\mathrm{n}}=\mathrm{p}_{0}+\mathrm{p}_{1} z+\mathrm{p}_{2} z^{2}+\mathrm{p}_{3} z^{3}+\ldots+\mathrm{p}_{1+2 s} z^{1+2 s},
$$

then at least one of the coefficients $\mathrm{p}_{0}, \mathrm{p}_{1}, \mathrm{p}_{2}, \mathrm{p}_{3}$ is different from zero.

Proof The proof is similar to the proof of Corollary 1 and as such it is omitted.

The families E7 and E3 
The confluent Heun equation associated with the family E7 is (equation (259))

$$
z(z-1) \mathrm{p}^{\prime \prime}(z)+\left(1-4 z s+2 z^{2} s\right) \mathrm{p}^{\prime}(z)+\left(-l(l+1)+2 s-4 s^{2} z\right) \mathrm{p}(z)=0 .
$$

Comparison with the general form of the confluent Heun equation (264) gives

$$
a=2 s, b=-4 s, c=1, d=-l(l+1)+2 s, \text { and } e=-4 s^{2} .
$$

According to the equivalence relation (269), the substitution

$$
\mathrm{p}(z)=z^{2} \mathrm{p}_{1}(z)
$$

into equation (259) yields

$z(z-1) \mathrm{p}_{1}{ }^{\prime \prime}(z)+\left(-3-2 z(2 s-2)+2 z^{2} s\right) \mathrm{p}_{1}^{\prime}(z)+(-l(l+1)-6 s+2-4 z s(2 s-2)) \mathrm{p}_{1}(z)=0$

which is nothing but the confluent Heun equation associated with the family E3 (equation (261)).

In subsection VIIID we give strong evidence which supports the thesis that the families E7 and E3 do not give rise to Liouvillian solutions to the master equation (66). Remarkably the equivalence of the families E7 and E3, shown by the substitution (277) into equation (259), enables us to reduce the consideration of the two cases E7 and E3 into the consideration of the case E7 only. This is precisely the content of the next Corollary.

Corollary 3 In order to prove that the the family E3 does not give rise to a Liouvillian solution to the master equation (6) it suffices to prove that the family E7 does not give rise to a Liouvillian solution to the master equation (6).

Proof This is an immediate consequence of the equivalence of the families E7 and E3. Equation (277) implies that if the confluent Heun equation (261), associated with the family E3, admits a polynomial solution $\mathrm{p}_{1}(\mathrm{z})$, then the confluent Heun equation (259), associated with the family $\mathrm{E} 7$, admits also a polynomial solution $\mathrm{p}(z)=z^{2} \mathrm{p}_{1}(z)$. Thus if equation (259) does not have a polynomial solution, and therefore, the family E7 does not give rise to a Liouvillian solution to the master equation (6), then equation (261) does not have a polynomial solution either, and therefore, the family E3 does not give rise to a Liouvillian solution to the master equation (6). This completes the proof. 


\section{The family S3 does not give rise to a Liouvillian solution}

For the family S3 we prove the following

Theorem 6 The family S3 does not give rise to a Liouvillian solution to the master equation (6).

Proof A Liouvillian solution arises from the family S3 if there is polynomial solution $\mathrm{P}$ to equation (73)

$$
\mathrm{P}^{\prime \prime}+2 \theta \mathrm{P}^{\prime}+\left(\theta^{2}+\theta^{\prime}-\nu\right) \mathrm{P}=0
$$

which in this case (equations (45) and (68) with $\beta=1$ ) yields

$$
\mathrm{P}^{\prime \prime}(r)+\frac{s r^{2}-4 r s+2 r-2}{r(r-2)} \mathrm{P}^{\prime}(r)+\frac{-r s(2 s-1)-l(l+1)-2 s}{r(r-2)} \mathrm{P}(r)=0 .
$$

The last equation has two regular singular points at $r=0, r=2$, and, one irregular singular

point of Poincare rank 1 at $r=\infty$. The roots of the associated indicial equations are the following

$$
\begin{array}{cc}
\text { Singular points } & \text { Roots } \\
r=0 & \rho=0 \text { (double root) }, \\
r=2 & \rho_{1}=0, \rho_{2}=2 s, \\
r=\infty & \rho=2 s-1 .
\end{array}
$$

One can easily check that if equation (278) admits a polynomial solution at all then this solution will have degree $2 s-1$, which is precisely the degree determined by Kovacic's algorithm. One can easily check that equation (278) cannot admit polynomial solution of zero degree. Thus in this proof we will assume hereafter that $2 s$ is an integer which takes values in the set $\{2,3,4, \ldots\}$.

According to Fuchs' Theorem a basis of the space of solutions of equation (278) in the interval $(0,2)$, when the indicial equation associated to the singular point $r=0$ has a double root $\rho=0$, is given by

$$
\begin{aligned}
& p_{1}(r)=\sum_{\mathrm{n}=0}^{\infty} \mathrm{P}_{\mathrm{n}} r^{\mathrm{n}}, \quad \mathrm{P}_{0} \neq 0, \quad \text { and } \\
& p_{2}(r)=\sum_{\mathrm{n}=0}^{\infty} \mathrm{k}_{\mathrm{n}} r^{\mathrm{n}}+p_{1} \ln r, \quad \mathrm{k}_{0} \neq 0 .
\end{aligned}
$$


By substituting $\mathrm{P}(r)=\sum_{\mathrm{n}=0}^{+\infty} \mathrm{P}_{\mathrm{n}} \mathrm{r}^{\mathrm{n}}$ into equation (278), we find that the coefficients $\mathrm{P}_{\mathrm{n}}$ satisfy the following three-term recurrence relation

$$
\begin{aligned}
& s(\mathrm{n}-2 s) \mathrm{P}_{\mathrm{n}-1}+\left(\mathrm{n}^{2}+\mathrm{n}-4 s \mathrm{n}-l(l+1)-2 s\right) \mathrm{P}_{\mathrm{n}} \\
& -2(\mathrm{n}+1)^{2} \mathrm{P}_{\mathrm{n}+1}=0, \quad \text { where, } \quad \mathrm{P}_{-1}=0 .
\end{aligned}
$$

Equation (281) gives the three-term recurrence relation satisfied by the coefficients $\mathrm{P}_{\mathrm{n}}$ of the power series solution $p_{1}(r)=\sum_{\mathrm{n}=0}^{\infty} \mathrm{P}_{\mathrm{n}} r^{\mathrm{n}}, \mathrm{P}_{0} \neq 0$, given by equation (279).

If equation (278) admits a polynomial solution $\mathcal{P}(r)=\sum_{\mathrm{n}=0}^{2 s-1} \mathrm{P}_{\mathrm{n}} \mathrm{r}^{\mathrm{n}}$ then this solution in the interval $(0,2)$ will admit an expansion in terms of $p_{1}(r)$ and $p_{2}(r)$ which span the solution space in this interval. Therefore

$$
\mathcal{P}(r)=\alpha p_{1}(r)+\beta p_{2}(r)
$$

for some $\alpha, \beta \in R$. The functional forms (279) and (280) of $p_{1}(r)$ and $p_{2}(r)$ respectively imply that $\beta=0$ and that $p_{1}(r)$ has to truncate so that to give a polynomial solution of degree $2 s-1$. Therefore

$$
\mathcal{P}(r)=\alpha \sum_{\mathrm{n}=0}^{2 s-1} \mathrm{P}_{\mathrm{n}} r^{\mathrm{n}}, \quad \mathrm{P}_{0} \neq 0,
$$

for some $\alpha \in R$.

The coefficients $\mathrm{P}_{\mathrm{n}}$ of the power series solution $p_{1}(r)$ (equation (279)) satisfy the three-term recurrence relation (281). In particular the three-term recurrence relation (281) for $\mathrm{n}=2 s-1$ and for $\mathrm{n}=2 s$ gives respectively

$$
\begin{gathered}
-s \mathrm{P}_{2 s-2}-\left(l(l+1)+4 s^{2}\right) \mathrm{P}_{2 s-1}-8 s^{2} \mathrm{P}_{2 s}=0, \quad \text { and } \\
-\left(l(l+1)+4 s^{2}\right) \mathrm{P}_{2 s}-2(2 s+1)^{2} \mathrm{P}_{2 s+1}=0 .
\end{gathered}
$$

From equations (284), (285) and the fact that the coefficient $-2(\mathrm{n}+1)^{2}$ of $\mathrm{P}_{\mathrm{n}+1}$ in the three-term recurrence relation (281) is different from zero for every n, and in particular for every $\mathrm{n}=2 s+1,2 s+2, \ldots$, we infer that the power series solution $p_{1}(r)$, given by equation (279), does indeed terminate and gives a polynomial solution of degree $2 s-1$ if and only if

$$
-s \mathrm{P}_{2 s-2}-\left(l(l+1)+4 s^{2}\right) \mathrm{P}_{2 s-1}=0 .
$$

In this case the coefficients of the polynomials $p_{1}(r)$ and of $\mathcal{P}(r)$ are proportional as equation (283) implies. In any case the coefficients of $\mathcal{P}(r)$ can only be determined up to a 
multiplicative constant. Equation (286) gives

$$
\frac{\mathrm{P}_{2 s-1}}{\mathrm{P}_{2 s-2}}=-\frac{s}{l(l+1)+4 s^{2}} \text {. }
$$

Moreover, according to Fuchs' Theorem, another basis of the space of solutions of equation (278) in the punctured neighbourhood $(0,2) \cup(2,4)$ of 2 , when the difference $\rho_{2}-\rho_{1}=2 s-0=$ $2 s \in\{2,3,4, \ldots\}$ of the roots $\rho_{1}, \rho_{2}$ of the indicial equation associated to the singular point $r=2$ is a positive integer, is given by

$$
\begin{aligned}
& p_{3}(r)=(r-2)^{2 s} \sum_{\mathrm{n}=0}^{\infty} P_{\mathrm{n}}(r-2)^{\mathrm{n}}, \quad P_{0} \neq 0, \quad \text { and }, \\
& p_{4}(r)=\sum_{\mathrm{n}=0}^{\infty} \mathrm{m}_{\mathrm{n}}(r-2)^{\mathrm{n}}+\mathrm{C} p_{1} \ln |r-2|, \quad \mathrm{m}_{0} \neq 0 .
\end{aligned}
$$

The constant $\mathrm{C}$ may turn out to be zero.

By substituting $\mathrm{P}(r)=(r-2)^{\rho} \sum_{\mathrm{n}=0}^{+\infty} P_{\mathrm{n}}(r-2)^{\mathrm{n}}$ into equation (278), where $\rho=0$ or $\rho=2 s$, we find that the coefficients $P_{\mathrm{n}}$ satisfy the following three-term recurrence relation

$$
\begin{aligned}
& s(\mathrm{n}+\rho-2 s) P_{\mathrm{n}-1}+\left((\mathrm{n}+\rho)(\mathrm{n}+\rho+1-2 s)-l(l+1)-4 s^{2}\right) P_{\mathrm{n}} \\
& +(\mathrm{n}+\rho+1)(2(\mathrm{n}+\rho+1)-4 s) P_{\mathrm{n}+1}=0, \quad \text { where, } \quad P_{-1}=0 .
\end{aligned}
$$

When $\rho=2 s$ equation (290) gives the three-term recurrence relation satisfied by the coefficients $P_{\mathrm{n}}$ of the power series solution $p_{3}(r)=(r-2)^{2 s} \sum_{\mathrm{n}=0}^{\infty} P_{\mathrm{n}}(r-2)^{\mathrm{n}}, P_{0} \neq 0$, given by equation (288). When $\rho=0$ equation (290) gives the three-term recurrence relation satisfied by the coefficients $\mathrm{m}_{\mathrm{n}}$ of the solution $p_{4}(r)=\sum_{\mathrm{n}=0}^{\infty} \mathrm{m}_{\mathrm{n}}(r-2)^{\mathrm{n}}+\mathrm{C} p_{1} \ln |r-2|, \mathrm{m}_{0} \neq 0$, given by equation (289), only when $\mathrm{C}=0$. When $\mathrm{C} \neq 0$ the coefficients $\mathrm{m}_{\mathrm{n}}$ of the power series $\sum_{\mathrm{n}=0}^{\infty} \mathrm{m}_{\mathrm{n}}(r-2)^{\mathrm{n}}$ appearing in the solution $p_{4}(r)$, given by equation (289), are determined by substituting $p_{4}(r)$ into equation (278).

The polynomial solution $\mathcal{P}(r)=\sum_{\mathrm{n}=0}^{2 s-1} \mathrm{P}_{\mathrm{n}} \mathrm{r}^{\mathrm{n}}$ will also admit an expansion in terms of $p_{3}(r)$ and $p_{4}(r)$ in the interval $(0,2)$, since $p_{3}(r)$ and $p_{4}(r)$ also span the solution space in this interval. Therefore

$$
\mathcal{P}(r)=\gamma p_{3}(r)+\delta p_{4}(r)
$$

for some $\gamma, \delta \in R$. The functional forms (288) and (289) of $p_{3}(r)$ and $p_{4}(r)$ imply that $\gamma=0$, $\mathrm{C}=0$, and that the power series $\sum_{\mathrm{n}=0}^{\infty} \mathrm{m}_{\mathrm{n}}(r-2)^{\mathrm{n}}, \mathrm{m}_{0} \neq 0$, which appears in $p_{4}(r)$ has to truncate so that to give a polynomial solution of degree $2 s-1$. Therefore

$$
\mathcal{P}(r)=\delta \sum_{\mathrm{n}=0}^{2 s-1} \mathrm{~m}_{\mathrm{n}}(r-2)^{\mathrm{n}}, \mathrm{m}_{0} \neq 0,
$$


for some $\delta \in R$. The coefficients $P_{\mathrm{n}}$ of the power series solution $p_{3}(r)$ (equation (288)) satisfy the three-term recurrence relation (290) when $\rho=2 s$. When $\mathrm{C}=0$ the coefficients $\mathrm{m}_{\mathrm{n}}$ of the power series $\sum_{\mathrm{n}=0}^{\infty} \mathrm{m}_{\mathrm{n}}(r-2)^{\mathrm{n}}, \mathrm{m}_{0} \neq 0$, satisfy also the three-term recurrence relation (290) with $\rho=0$.

In particular the three-term recurrence relation (290) for $\mathrm{n}=2 s-1$ and for $\mathrm{n}=2 s$, when $\rho=0$, gives respectively

$$
\begin{aligned}
-s \mathrm{~m}_{2 s-2}-\left(l(l+1)+4 s^{2}\right) \mathrm{m}_{2 s-1}+0 \mathrm{~m}_{2 s} & =0, \quad \text { and } \\
0 \mathrm{~m}_{2 s-1}-(l(l+1)+2 s(2 s-1)) \mathrm{m}_{2 s}+2(2 s+1) \mathrm{m}_{2 s+1} & =0
\end{aligned}
$$

The following two remarks are now in order

1. The coefficients of $m_{n-1}, m_{n}$ and of $m_{n+1}$ of the three-term recurrence relation (290) contain $\mathrm{n}$ only when $\mathrm{n}$ is added to $\rho$. Consequently the coefficients of the relations resulting from (290) when $\rho=0$ and $\mathrm{n}=2 s, \mathrm{n}=2 s+1, \mathrm{n}=2 s+2$, and $\mathrm{n}=2 s+3, \ldots$, are the same with the coefficients of the relations resulting from (290) when $\rho=2 s$ and $\mathrm{n}=0, \mathrm{n}=1, \mathrm{n}=2$, and $\mathrm{n}=3, \ldots$.

2. Both the coefficient of $m_{2 s}$ in (293) and the coefficient of $m_{2 s-1}$ in (294) are zero.

From remarks 1 and 2 and equations (293) and (294) we conclude that when $\mathrm{C}=0$ we have

$$
p_{4}(r)=\sum_{\mathrm{n}=0}^{\infty} \mathrm{m}_{\mathrm{n}}(r-2)^{\mathrm{n}}=\sum_{\mathrm{n}=0}^{2 s-1} \mathrm{~m}_{\mathrm{n}}(r-2)^{\mathrm{n}}+p_{3}(r), \mathrm{m}_{0} \neq 0 .
$$

Since $p_{3}(r)$ is a solution to equation (278), and equation (278) is linear, the polynomial $\sum_{\mathrm{n}=0}^{2 s-1} \mathrm{~m}_{\mathrm{n}}(r-2)^{\mathrm{n}}$ of degree $2 s-1$ is also a solution to equation (278). This explains how the polynomial solution

$$
\mathcal{P}(r)=\delta \sum_{\mathrm{n}=0}^{2 s-1} \mathrm{~m}_{\mathrm{n}}(r-2)^{\mathrm{n}}, \mathrm{m}_{0} \neq 0,
$$

(equation (292)), if it exists, is derived from the basis $p_{3}(r), p_{4}(r)$, given by equations (288) and (289), spanning the solution space of equation (278) in the the punctured neighbourhood $(0,2) \cup(2,4)$ of 2 . We note that equation (293) gives

$$
\frac{\mathrm{m}_{2 s-1}}{\mathrm{~m}_{2 s-2}}=-\frac{s}{l(l+1)+4 s^{2}} .
$$

The key observation is that, according to Fuchs' Theorem, in the interval $(0,2)$, both $p_{1}(r), p_{2}(r)$, given by equations (279), (280) respectively, and $p_{3}(r), p_{4}(r)$, given by equations 
(288), (289) correspondingly, constitute a basis of the space of solutions of equation (278). Thus in the interval $(0,2)$ both equations (283) and (292) hold simultaneously. Therefore in the interval $(0,2)$ we obtain

$$
\mathcal{P}(r)=\alpha \sum_{\mathrm{n}=0}^{2 s-1} \mathrm{P}_{\mathrm{n}} r^{\mathrm{n}}=\delta \sum_{\mathrm{n}=0}^{2 s-1} \mathrm{~m}_{\mathrm{n}}(r-2)^{\mathrm{n}}, \quad \mathrm{P}_{0} \neq 0, \mathrm{~m}_{0} \neq 0
$$

where $\alpha, \delta$ are non zero real numbers.

From equation (297), by equating the coefficients of $r^{2 s-1}$ and $r^{2 s-2}$, we obtain

$$
\begin{aligned}
& \alpha \mathrm{P}_{2 s-1}=\delta \mathrm{m}_{2 s-1}, \\
& \alpha \mathrm{P}_{2 s-2}=\delta\left((-2)\left(\begin{array}{l}
2 s-1 \\
2 s-2
\end{array}\right) \mathrm{m}_{2 s-1}+\mathrm{m}_{2 s-2}\right) .
\end{aligned}
$$

Division of equations (298) and (299) by parts yields

$$
\frac{\mathrm{P}_{2 s-1}}{\mathrm{P}_{2 s-2}}=\frac{1}{2(1-2 s)+\frac{\mathrm{m}_{2 s-2}}{\mathrm{~m}_{2 s-1}}} .
$$

By combining equations (287), (296) and (300) we have

$$
s=0 \text { or } s=\frac{1}{2}
$$

Therefore the compatibility condition (297), which must be satisfied if a polynomial solution to equation (278) exists, can be satisfied if and only if $s=0$ or $s=\frac{1}{2}$. However, when $s=0$ the degree $\mathrm{d}_{\mathrm{s}}=2 s-1$ of the sought polynomial becomes negative, and when $s=\frac{1}{2}$ the degree $\mathrm{d}_{\mathrm{s}}$ of the sought polynomial becomes zero. One can easily check that equation (278) does not admit polynomial solution of zero degree when $s=\frac{1}{2}$. Consequently the requirement that the compatibility condition (297) must be satisfied leads to a contradiction. We conclude that there is no polynomial solution to equation (278). This completes the proof.

The method employed in the proof of Theorem [ applied to the other remaining cases G3, E3 and E7 does not lead to contradictions, as it happens in the case of the family S3, but leads instead to trivial identities, and thus no conclusions can be drawn for the families G3, E3 and E7 by applying the method employed in the proof of Theorem 6. For the families G3, E3 and E7, in the next subsection, we give strong evidence which supports the thesis that the families G3, E3 and E7 do not give rise to Liouvillian solutions to the master equation (6). 


\section{Evidence for the families G3, E3 and E7}

In this subsection we give strong evidence which supports the thesis that the families G3, E3 and E7 do not give rise to Liouvillian solutions to the master equation (6).

We start with the family G3. The family G3 will give rise to a Liouvillian solution to the master equation (6) if there is polynomial solution $\mathrm{P}$ to equation (73)

$$
\mathrm{P}^{\prime \prime}+2 \theta \mathrm{P}^{\prime}+\left(\theta^{2}+\theta^{\prime}-\nu\right) \mathrm{P}=0
$$

where, (equation (65)),

$$
\theta(r)=\frac{5 / 2}{r}+\frac{\frac{1}{2}-s}{r-2}+\frac{s}{2}
$$

and, (equation (45) ),

$$
\nu(r)=\frac{\frac{s^{2}}{4} r^{4}+l(l+1) r^{2}+2[\beta-l(l+1)-1] r+3-4 \beta}{r^{2}(r-2)^{2}},
$$

where, $\beta=-3$. One easily finds that if a a polynomial solution $\mathrm{P}$ to equation (73) exists at all, then this solution will have degree $\mathrm{d}=2 s-3$; this is precisely the degree of the sought polynomial dictated by Kovacic's algorithm.

By substituting $\mathrm{P}(r)=\sum_{\mathrm{n}=0}^{2 s-3} \mathrm{P}_{\mathrm{n}} r^{\mathrm{n}}$ into equation (73) we obtain that the following 3 -term recurrence relation holds for the coefficients $\mathrm{P}_{\mathrm{n}}$

$$
-s(2 s-2-\mathrm{n}) \mathrm{P}_{\mathrm{n}-1}+\left(\mathrm{n}^{2}+5 \mathrm{n}-4 s \mathrm{n}+6-l(l+1)-10 s\right) \mathrm{P}_{\mathrm{n}}-2(\mathrm{n}+1)(\mathrm{n}+5) \mathrm{P}_{\mathrm{n}+1}=0,
$$

where, $\mathrm{n} \in\{0,1,2, \ldots, 2 s-3\}$, and, $\mathrm{P}_{-1}=\mathrm{P}_{2 s-2}=0$.

We find that for polynomials of degree $\mathrm{d}=0, \ldots, 500$ the determinants $\mathrm{D}_{\mathrm{d}+1}$ of the coefficients of the associated $(d+1) \times(d+1)$ linear homogeneous systems are either strictly positive or strictly negative. In particular,

$$
\operatorname{sgn}\left(\mathrm{D}_{\mathrm{d}+1}\right)=(-1)^{\mathrm{d}+1}, \quad \mathrm{~d}=0,1,2, \ldots, 500 .
$$

We stopped at $\mathrm{d}=500$ because the required time for the calculation of determinants of matrices of order $n \times n$, with computers of medium performance, tends to become large when $\mathrm{n}$ approaches and exceeds the value 500 .

In general, from equation (302) we obtain that the determinant of the associated (2s$2) \times(2 s-2)$ linear homogeneous system is tridiagonal and has the form 


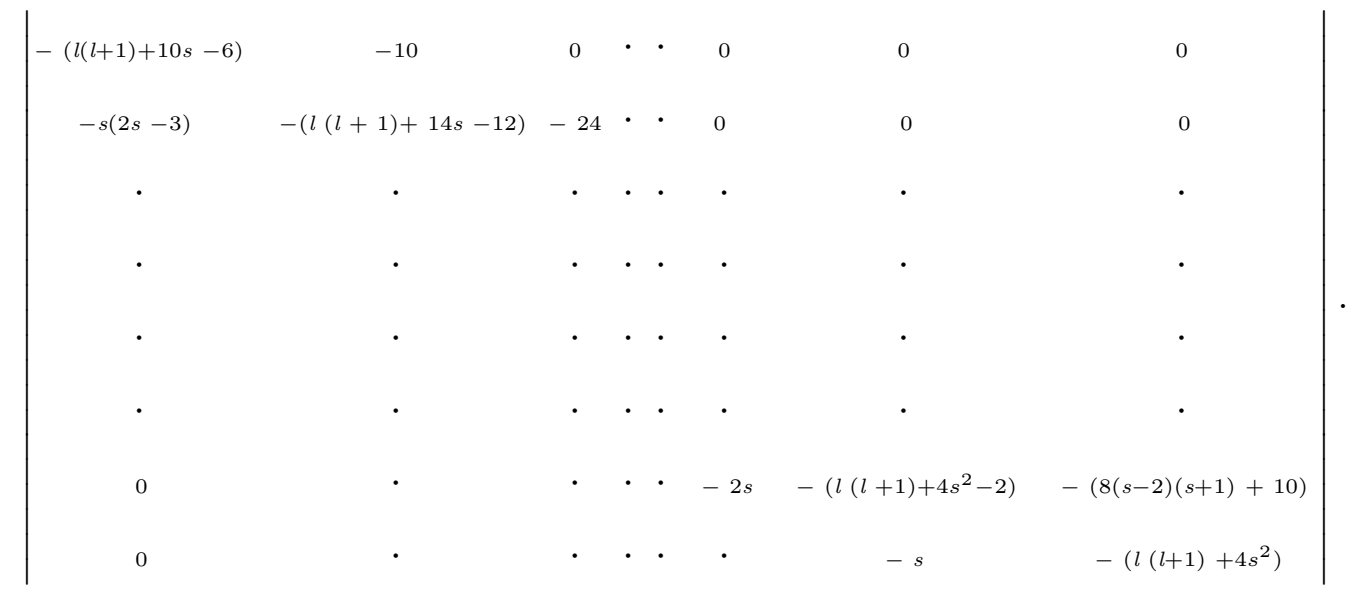

Equation (303) suggests that the determinant of the aforementioned tridiagonal matrix is different from zero for any value of $\mathrm{d}$. By expanding along the last column we find that this determinant satisfies the following 3 -term recurrence relation

$$
\mathrm{D}_{\mathrm{n}+1}=\left(\mathrm{n}^{2}+5 \mathrm{n}-4 s \mathrm{n}+6-l(l+1)-10 s\right) \mathrm{D}_{\mathrm{n}}-2 \mathrm{n} s(\mathrm{n}+4)(2 s-2-\mathrm{n}) \mathrm{D}_{\mathrm{n}-1},
$$

where it is understood that $\mathrm{n}=2 s-3, \mathrm{n} \in\{0,1,2, \ldots\}, \mathrm{D}_{0}=1$, and $\mathrm{D}_{-1}=0$.

We examine the similarly the remaining families cases E3 and E7. By substituting $\mathrm{P}(r)=$ $\sum_{\mathrm{n}=0}^{2 s-2} \mathrm{P}_{\mathrm{n}} r^{\mathrm{n}}$, and, $\mathrm{P}(r)=\sum_{\mathrm{n}=0}^{2 s} \mathrm{P}_{\mathrm{n}} r^{\mathrm{n}}$ into equation (73) we obtain that the coefficients $\mathrm{P}_{\mathrm{n}}$ satisfy respectively the following 3 -term recurrence relations

$$
-s(2 s-1-\mathrm{n}) \mathrm{P}_{\mathrm{n}-1}+\left(\mathrm{n}^{2}+3 \mathrm{n}-4 s \mathrm{n}+2-l(l+1)-6 s\right) \mathrm{P}_{\mathrm{n}}-2(\mathrm{n}+1)(\mathrm{n}+3) \mathrm{P}_{\mathrm{n}+1}=0,
$$

where, $\mathrm{n} \in\{0,1,2, \ldots, 2 s-2\}$, and, $\mathrm{P}_{-1}=\mathrm{P}_{2 s-1}=0$,

$$
-s(2 s+1-\mathrm{n}) \mathrm{P}_{\mathrm{n}-1}+\left(\mathrm{n}^{2}-\mathrm{n}-4 s \mathrm{n}-l(l+1)+2 s\right) \mathrm{P}_{\mathrm{n}}-2(\mathrm{n}+1)(\mathrm{n}-1) \mathrm{P}_{\mathrm{n}+1}=0,
$$

where, $\mathrm{n} \in\{0,1,2, \ldots, 2 s\}$, and, $\mathrm{P}_{-1}=\mathrm{P}_{2 s+1}=0$.

We find again that for polynomials of degree $d=0, \ldots, 500$ the determinants $D_{d+1}$ of the coefficients of the associated $(\mathrm{d}+1) \times(\mathrm{d}+1)$ linear homogeneous systems are either strictly positive or strictly negative, i.e., we find that equation (303) holds in all the remaining cases G3, E3 and E7.

From equations (306) and (307) we obtain that the determinants $\mathrm{D}_{\mathrm{n}}$ of the associated linear homogeneous systems are tridiagonal and have respectively the form

$$
\begin{aligned}
\mathrm{d}_{(\mathrm{i}+1)(\mathrm{i})} & =-s(2 s-1-\mathrm{i}), \\
\mathrm{d}_{(\mathrm{i}+1)(\mathrm{i}+1)} & =\left(\mathrm{i}^{2}+3 \mathrm{i}-4 s \mathrm{i}+2-l(l+1)-6 s\right), \\
\mathrm{d}_{(\mathrm{i}+1)(\mathrm{i}+2)} & =-2(\mathrm{i}+1)(\mathrm{i}+3),
\end{aligned}
$$


where, $\mathrm{i} \in\{0,1,2, \ldots, 2 s-2\}$,

$$
\begin{aligned}
\mathrm{d}_{(\mathrm{i}+1)(\mathrm{i})} & =-s(2 s+1-\mathrm{i}), \\
\mathrm{d}_{(\mathrm{i}+1)(\mathrm{i}+1)} & =\left(\mathrm{i}^{2}-\mathrm{i}-4 s \mathrm{i}-l(l+1)+2 s\right), \\
\mathrm{d}_{(\mathrm{i}+1)(\mathrm{i}+2)} & =-2(\mathrm{i}+1)(\mathrm{i}-1),
\end{aligned}
$$

where, $\mathrm{i} \in\{0,1,2, \ldots, 2 s\}$. In both cases $\mathrm{d}_{(\mathrm{k})(\mathrm{j})}$ denotes the element of $\mathrm{D}_{\mathrm{n}}$ which belongs to the row $\mathrm{k}$ and the column $\mathrm{j}$.

By expanding the previous determinants along their last columns we find that they satisfy respectively the following 3 -term recurrence relations

$$
\mathrm{D}_{\mathrm{n}+1}=\left(\mathrm{n}^{2}+3 \mathrm{n}-4 s \mathrm{n}+2-l(l+1)-6 s\right) \mathrm{D}_{\mathrm{n}}-2 \mathrm{n} s(\mathrm{n}+2)(2 s-1-\mathrm{n}) \mathrm{D}_{\mathrm{n}-1},
$$

where it is understood that $\mathrm{n}=2 s-2, \mathrm{n} \in\{0,1,2, \ldots\}, \mathrm{D}_{0}=1$, and $\mathrm{D}_{-1}=0$, and,

$$
\mathrm{D}_{\mathrm{n}+1}=\left(\mathrm{n}^{2}-\mathrm{n}-4 s \mathrm{n}-l(l+1)+2 s\right) \mathrm{D}_{\mathrm{n}}-2 \mathrm{n} s(\mathrm{n}-2)(2 s+1-\mathrm{n}) \mathrm{D}_{\mathrm{n}-1},
$$

where it is understood that $\mathrm{n}=2 s, \mathrm{n} \in\{0,1,2, \ldots\}, \mathrm{D}_{0}=1, \mathrm{D}_{-1}=0$.

Equation (303) suggests that the remaining families G3, E3, E7 do not give any new Liouvillian solutions. Proving this amounts to proving that equations (73) in the remaining families do not have any polynomial solutions. This in fact is an intriguing and difficult problem and can be cast into different forms. To mention a few:

1. Employing (305), (310), (311), and the properties of the tridiagonal determinants, to prove that equation (303) holds for any value of $\mathrm{d}$.

2. Using (305), (310), (311), and the properties of continued fractions, to prove that equation (303) holds for any value of $\mathrm{d}$.

3. Proving that the 3 -term recurrence relations (302), (306), and (307) do not terminate.

4. Extending (73) to the complex plane and studying its monodromy group in order to prove that the existence of polynomial solutions is not compatible with the structure of its singularities.

The main difficulty in approaches 1,2 and to a lesser extent in 3, which complicates the analysis considerably, is that in all the remaining families G3, E3, and E7, the order 
of the associated tridiagonal determinants increases with the degree of the corresponding polynomial, e.g., in the family E7 the degree of the polynomial $\mathrm{P}(r)=\sum_{\mathrm{n}=0}^{2 s} \mathrm{P}_{\mathrm{n}} r^{\mathrm{n}}$ increases with the order of the determinant (309).

All approaches 1, 2, 3, 4, and variations thereof, were attempted with various degrees of success providing very useful insight into the nature of the problem but without any definite result.

\section{E. Reduction of the four remaining families to two families and a Conjecture}

Kovacic's algorithm (section III, Theorem 11) constructs all the Liouvillian solutions of equation $y^{\prime \prime}(x)=\nu(x) y(x)$ (equation (20)),$\nu(x)$ is a rational function over the field of complex numbers, in the form $\eta(x)=e^{\int \omega(x) \mathrm{d} x}$, where $\omega(x)$ is an algebraic function of $x$ of degree $\mathrm{n}$ which in general takes the values $1,2,4,6$ or 12 . The algebraic function $\omega(x)$ solves an irreducible algebraic equation $\Pi(\omega, x)=\sum_{\mathrm{i}=0}^{\mathrm{n}} \frac{\mathrm{P}_{\mathrm{i}}(x)}{(\mathrm{n}-\mathrm{i})} \omega^{\mathrm{i}}=0$, where $\mathrm{P}_{\mathrm{i}}(x)$ are rational functions of $x$, (equation (19)) .

The search of Kovacic's algorithm for $\Pi(\omega, x)$ is based on the knowledge of the poles of $\nu$ and consists in constructing and testing a finite number of possible candidates for $\Pi(\omega, x)$. If no $\Pi(\omega, x)$ is found then the differential equation (20) has no Liouvillian solutions. For each n Kovacic's algorithm constructs a different finite set of possible candidates for $\Pi(\omega, x)$.

Each candidate $\Pi(\omega, x)$, in particular the coefficients $\frac{\mathrm{P}_{\mathrm{i}}(x)}{(\mathrm{n}-\mathrm{i}) !}$ of the algebraic equation $\Pi(\omega, x)=0$, depend on a rational function $\theta(x)$ and an associated polynomial $\mathrm{P}$ of degree $\mathrm{d}$ which solves an ODE with rational function coefficients of order $n+1$. The coefficients of this ODE depend on $\theta$ and on $\nu$. For convenience we call a pair $(\mathrm{d}, \theta)$, a family.

To conclude the degree $\mathrm{n}$ of the algebraic function $\omega(x)$ depends on the structure of poles of $\nu(x)$ and can take the values 1,2,4,6 and 12. For each value of $\mathrm{n}$ Kovacic's algorithm constructs a finite set of families $(\mathrm{d}, \theta)$. For each family $(\mathrm{d}, \theta)$ Kovacic's algorithm constructs an ODE of order $\mathrm{n}+1$. If this ODE has a polynomial solution $\mathrm{P}$ of degree $\mathrm{d}$ then Kovacic's algorithm constructs, from this polynomial $\mathrm{P}$, an algebraic function $\omega(x)$, of degree $\mathrm{n}$, which solves the associated algebraic equation $\Pi(\omega, x)=0$.

Therefore, with running the risk of oversimplification, Kovacic's algorithm reduces the problem of finding Liouvillian solutions to equation (20), to the problem of finding polynomial solutions to an associated set $\mathcal{D}$ of linear ODEs whose coefficients are rational functions 
over the field of complex numbers.

In section IV we showed, that in the problem we are examining in this paper, the structure of poles of the master equation (6) dictates that the possible values of the degree $\mathrm{n}$ of the sought algebraic functions $\omega(x)$ are 1 and 2. In section IV we gave explicitly, in detail, the families $(\mathrm{d}, \theta)$ constructed by Kovacic's algorithm, when $\mathrm{n}=1$, and we showed that the only families remaining for consideration, when $\mathrm{n}=1$, are the families named G3, G7, G8, E3, E7, and S3.

In subsection $\mathrm{VB}$ we considered the family $\mathrm{G} 8$. In subsection $\mathrm{VC}$ we considered the family G7. Therefore the results of the section [V] and the results of the subsections $\mathrm{VB}$ and $\mathrm{VC}$ show that, the only families remaining for consideration, when $\mathrm{n}=1$, are the families G3, E3, E7, and S3. In this section we show that the four families G3, E3, E7, and S3 are reduced into two families G3 and E7. The case $n=2$ is only treated in appendix $\mathrm{A}$ where we show that, when $\mathrm{n}=2$, there are no families to consider.

We now explain how the four families G3, E3, E7, and S3 are reduced into two families G3 and E7. In subsection VIIIB, in Corollary 3, we showed that the examination of the two cases E3 and E7 can be reduced into the examination of the case E7 only: In order to prove that the the family E3 does not give rise to a Liouvillian solution to the master equation (6) it suffices to prove that the family E7 does not give rise to a Liouvillian solution to the master equation (6).

In subsection VIIIC we showed that the family S3 does not give rise to a Liouvillian solution to the master equation (6). Therefore the four remaining families G3, E3, E7, and S3 are reduced into two families, the families G3 and E7. In subsection VIIID we gave strong evidence which supports the thesis that the families G3, E7 (and E3) do not give rise to Liouvillian solutions to the master equation ([6]).

Therefore it is tempting to put forward the following

Conjecture The families G3 and E7 do not give rise to Liouvillian solutions to the master equation (6). Consequently the only "closed-form" solutions to the equations describing scalar, electromagnetic and gravitational radiative perturbations on the static part of the Schwarzschild space-time are those found in the gravitational case, in subsections $V B$ and $\overline{V C}$, and which were initially found by Chandrasekhar 14 .

We can examine if the family G3 gives rise to Liouvillian solutions to the master equation 
(6) either by using the methods 1, 2, 3, and 4 given in subsection VIIID or/and by using the Corollaries 1 and 2 given in subsection VD.

\section{F. Comparison of our results with the results of Couch and Holder}

Couch and Holder $\underline{26}$ also consider problem 3 , in the list of most difficult problems, mentioned in subsection [A, which we have to solve when we apply Kovacic's algorithm: They give a partial proof that there are no families which give rise to Liouvillian solutions to the equation which governs the evolution of first order perturbations, scalar, electromagnetic, or gravitational, to the Schwarzschild geometry apart from those we found in subsections $\mathrm{VB}$ and $\mathrm{VC}$, and which were found initially by Chandrasekhar $\underline{14}$.

The main differences between our results on the solution of problem 3 and of the results derived by C.H. $\underline{\underline{26}}$ on the solution of the same problem are the following:

1. In subsection VIIIE we showed that when $n=1$ the only families remaining for consideration are the families G3 and E7. In subsection VIIID we gave strong evidence which supports the thesis that the families G3 and E7 do not give rise to Liouvillian solutions to the master equation (6). Thus, we conjectured in subsection VIIIE, that the only Liouvillian solutions to the master equation ([6) are the Liouvillian solutions found in subsections $\mathrm{VB}$ and $\mathrm{VC}$. In order to prove that the Conjecture is true we need to prove that the families G3 and E7 do not give rise to Liouvillian solutions to the master equation (6). On the other hand C.H. prove ${ }^{26}$, in their consideration of the problem, that when $\mathrm{n}=1$ no families give rise to Liouvillian solutions to the master equation which governs the evolution of the first order perturbations of the Schwarzschild geometry apart from the family which gives rise to the Liouvillian solution we found in subsection $\mathrm{VB}$,

2. In our consideration of the problem we are examining in this paper, we proved, in subsection $\overline{\nabla B}$, that, in the case of the gravitational perturbations of the Schwarzschild geometry, the family G8 gives rise to the Liouvillian solution $\chi$ to the master equation (6), and in subsection $\mathrm{VC}$, we proved that, the family G7 gives rise to the Liouvillian

solution $\chi \int \frac{\mathrm{d} r_{*}}{\chi^{2}}$ to the master equation (6) $)$. On the other hand in the consideration of the problem by C.H. $\underline{\underline{26}}$ there exists a family which gives rise to the Liouvillian solution 
$\chi$ but it appears, curiously enough, that there exists no family which gives rise to the Liouvillian solution $\chi \int \frac{\mathrm{d} r_{*}}{\chi^{2}}$. Consequently, needless to say, the proof we gave in subsection $\mathrm{VC}$ is totally absent in the consideration of the problem by C.H. ${ }^{26}$.

3. In appendix $\mathrm{A}$ we show that when $\mathrm{n}=2$ there are no remaining families which could give rise to Liouvillian solutions to the master equation (66). On the other hand C.H. show $^{26}$, in their consideration of the problem, that when $n=2$ there are remaining families which could give rise to Liouvillian solutions to the master equation which governs the evolution of first order perturbations of the Schwarzschild geometry. As we already have pointed out in section II, to a great extent C.H. present their results $\mathrm{in}^{26}$ in a succinct form. Due to the succinct form of the presentation of the results $\mathrm{in}^{26}$ it is not easy by studying ${ }^{26}$ to figure out how many families could give rise to Liouvillian solutions in the study of the problem by C.H. ${ }^{26}$ when $n=2$. In any case, irrespective of the number of remaining families which could give rise to Liouvillian solutions in ${ }^{26}$ when $\mathrm{n}=2$ it is not easy to prove or disprove that these remaining families do not give rise to Liouvillian solutions to the master equation which governs the evolution of first order perturbations of the Schwarzschild geometry. To quote C.H.26 “... apparently, it is not straightforward to see how to settle the question of existence of type 2 Liouvillian solutions in the Schwarzschild case". In the terminology advocated by C.H. "type 2 Liouvillian solutions" are the Liouvillian solutions obtained when $\mathrm{n}=2$.

In relation with the differences 1,2 , and 3, we note the following: C.H. do not start from the master equation (6). There is an alternative treatment of the first order perturbations of the Schwarzschild geometry, via the Newman-Penrose formalism (chapter 29 in $^{72}$ ), which leads to a differential equation (equation (1) in ${ }^{26}$ ) different from the master equation (6) ). For convenience, let us call $\mathcal{P} \mathcal{E}$ the differential equation derived via the Newman-Penrose formalism. The exact form of the differential equation $\mathcal{P} \mathcal{E}$ is not needed.

The following statement, made by C.H. ${ }^{26}$, on the relation between the master equation (6) , which we use as our point of departure, and the differential equation $\mathcal{P} \mathcal{E}$, which is used by C.H. ${ }^{26}$ as their point of departure, is relevant here.

"...The equations resulting from different approaches are related, essentially by linear differential operators. Several of these relations are given in Ref. $\stackrel{72}{2}$ and from them it is easy 
to show that every Liouvillian solution to $\mathcal{P} \mathcal{E}$ generates a Liouvillian solution to the related differential equation (6), and, conversely. We omit the details of the essentially bookkeeping argument which proves this. Hence by finding all Liouvillian solutions of $\mathcal{P} \mathcal{E}$ one finds the Liouvillian solutions in other characterizations of the perturbations."

This statement is correct but it is not evident which are its implications for the differences 1, 2, and 3, unless one repeats all over again the analysis of C.H. ${ }^{26}$. The main reasons are: 1) There is no bijective correspondence between the families which arise when we apply Kovacic's algorithm to the master equation (6) and the families which arise when we apply Kovacic's algorithm to the differential equation $\mathcal{P E}$, neither when $\mathrm{n}=1$, nor when $\mathrm{n}=2$. 2) When we apply Kovacic's algorithm to the differential equation $y^{\prime \prime}+a y^{\prime}+b y=0$, (equation 1), where $a$ and $b$ are rational functions of a complex variable $x$, it is not generally true $\mathrm{e}^{20}$ that if one of the retained families gives rise to a Liouvillian solution $\eta$ to equation (1) then another retained family will give rise to $\eta \int \frac{e^{-\int a}}{\eta^{2}}$.

However, the previous statement quoted by C.H. suggests that in the study of the problem by C.H. ${ }^{26}$ there ought to exist a retained family which gives rise to the Liouvillian solution $\chi \int \frac{\mathrm{d} r_{*}}{\chi^{2}}$. But such a family does not appear in 26 . So either this can be attributed to the remarks 1) and 2) of the previous paragraph or there is a flaw in the consideration of the problem by C.H. $\stackrel{26}{ }$. To decide between the two alternatives we need to repeat all over again the analysis of C.H..$^{26}$, since the succinct form of the presentation of the results in ${ }^{26}$ does not allow us to reach a conclusion on the cause of this discrepancy.

Moreover, the previous statement quoted by C.H. suggests that in our consideration of the problem the families G3 and E7 do not give rise to Liouvillian solutions to the master equation (6). Furthermore, the same statement, in the consideration of the problem by C.H. ${ }^{26}$, suggests that when $n=2$ the remaining families which could give rise to Liouvillian solutions to the differential equation $\mathcal{P} \mathcal{E}$ do not in fact do so.

This is in accord with the evidence given in subsection VIIID which supports the thesis that the families families G3 and E7 do not give rise to Liouvillian solutions to the master equation ([6), and also, it is in accord with the evidence given by C.H. ${ }^{26}$ that the remaining families when $\mathrm{n}=2$ do not in fact give rise to Liouvillian solutions to the differential equation $\mathcal{P E}$.

What is even more interesting is that we could in principle use the bijective correspondence between the Liouvillian solutions of the master equation (6) and the Liouvillian so- 
lutions of the differential equation $\mathcal{P} \mathcal{E}$ in order to prove that, in our consideration of the problem, the families G3 and E7 do not give rise to Liouvillian solutions to the master equation ([6), and also, in order to prove that, in the consideration of the problem by C.H. $\stackrel{26}{ }$, the remaining families when $n=2$ do not give rise to Liouvillian solutions to the differential equation $\mathcal{P} \mathcal{E}$. We expect that the proof will be in line with the proof of the Corollaries 1, 2 and 3. In order to examine if this is indeed the case we need again to repeat all over the analysis of Couch and Holder in ${ }^{26}$. We leave for future investigation the problem of repeating all over the analysis of Couch and Holder in ${ }^{26}$ and of clarifying with this analysis the issues raised before.

\section{The proof of Couch and Holder}

The nicest thing in ${ }^{26}$ is the proof given by Couch and Holder that when $n=1$ no families give rise to Liouvillian solutions to the differential equation $\mathcal{P} \mathcal{E}$ apart from the family which gives rise to the Liouvillian solution we found in subsection $\mathrm{VB}$. The proof starts with a clever rearrangement of the three-term recurrence relations associated with the remaining families and is done with induction. Interestingly enough, in our consideration of the problem, when we try to prove, by following the method of the aforementioned proof given by C.H. ${ }^{26}$, that the remaining families G3, E3, E7 and S3 do not give rise to Liouvillian solutions, we fail bluntly.

The failure is caused by the structure of the three-term recurrence relations associated with the remaining families G3, E3, E7 and S3. For this reason we need to employ different methods in order to prove that the remaining families do not give rise to Liouvillian solutions to the master equation (6). One such method is used in subsection VIIIC where we proved that the family S3 does not give rise to Liouvillian solution to the master equation (6).

Proving that a retained family does not give rise to a Liouvillian solution is one of the most difficult problems we have to solve when we apply Kovacic's algorithm. This is problem 3 , in the list of most difficult problems, mentioned in subsection IA, which we have to solve when we apply Kovacic's algorithm.

This in fact is an intriguing and difficult problem, it can be cast into different forms, and it can be approached with a variety of solution methods. Four such methods are the methods 1, 2, 3, and 4, given in subsection VIIID. Another method is the one we employ in 
subsection VIIIC, A concrete realization of the method 3 given in subsection VIIID is the method of proof given by C.H. $\underline{26}$.

A key observation regarding the work of C.H. is that at the very beginning of their study they use the transformation (14), which leaves equation $\mathcal{P E}$ quasi-invariant. C.H. apply Kovacic's algorithm to the differential equation $\mathcal{P} \mathcal{E}$ by taking into account its quasi-invariance under the transformation (14).

The quasi-invariance of $\mathcal{P E}$ implies in particular (we quote C.H. ${ }^{26}$ ) "So, without loss of generality, we on occasion take $M=1$ or $\frac{1}{2}$ in order to simplify expressions." Throughout their long paper $\underline{26}$ C.H. take $M=1$ with one single exception, their proof that no families, when $n=1$, give rise to Liouvillian solutions to the differential equation $\mathcal{P E}$, apart from the family which gives rise to the Liouvillian solution we found in subsection $\overline{V B}$, where they take $M=\frac{1}{2}$. It is highly likely that the choice $M=\frac{1}{2}$ does not just simplify expressions, but instead, it is absolutely essential for the proof given by C.H..

It is interesting and also useful for future applications of Kovacic's algorithm to know if this is indeed the case. In principle the proof of C.H. can be given for one single value of $M$, or for a range of values of $M$. This is allowed by the quasi-invariance of the differential equation $\mathcal{P E}$ under the transformation (14). A study of the results of C.H., in their present succinct form, does not allow us to understand if this is indeed the case. For this to happen we need to repeat the analysis of C.H. $\underline{\underline{26}}$ all over again.

It is also interesting and useful for future applications of Kovacic's algorithm to clarify why the method of proof given by C.H. $\underline{26}^{\underline{6}}$ fails, for any value of $M$, when we try to apply it to the families G3, E3, E7, and S3, which arise in our consideration of the problem when $\mathrm{n}=1$.

All the remaining families in C.H., when $\mathrm{n}=1$, are associated with three-term recurrence relations which have the general form

$$
\mathcal{C}_{\mathrm{k}-1}(s, \mathrm{k}, l, M) \mathrm{P}_{\mathrm{k}-1}+\mathcal{C}_{\mathrm{k}}(s, \mathrm{k}, l, M) \mathrm{P}_{\mathrm{k}}+\mathcal{C}_{\mathrm{k}+1}(s, \mathrm{k}, l, M) \mathrm{P}_{\mathrm{k}+1}=0,
$$

where the coefficients $\mathcal{C}_{\mathrm{k}-1}(s, \mathrm{k}, l, M), \mathcal{C}_{\mathrm{k}}(s, \mathrm{k}, l, M)$, and $\mathcal{C}_{\mathrm{k}+1}(s, \mathrm{k}, l, M)$, depend on the index $\mathrm{k}$, on the parameter $s=2 \mathrm{i} \sigma, \sigma$ is the frequency of the perturbations, on the angular harmonic index $l$, and on the mass $M$ of the black hole.

C.H. start their proof ${ }^{26}$ with the three-term recurrence relation (312). They take $M=\frac{1}{2}$, assume that the recurrence relation terminates to give a polynomial solution of degree $n$, 
and they reach a contradiction. In the following relations we do not set $M=\frac{1}{2}$ only to bring at home why the method of proof of C.H. ${ }^{26}$ fails when we try to apply it to the remaining families G3, E3, E7, and S3, which arise when $\mathrm{n}=1$, in our consideration of the problem. Thus in the rest of the proof $M$ takes the value $\frac{1}{2}$, although this is not explicitly stated.

If the three-term recurrence relation (312) terminates to give a polynomial solution of degree $\mathrm{n}$, then, the coefficients $\mathcal{C}_{\mathrm{k}-1}(s, \mathrm{k}, l, M), \mathcal{C}_{\mathrm{k}}(s, \mathrm{k}, l, M)$, and $\mathcal{C}_{\mathrm{k}+1}(s, \mathrm{k}, l, M)$, are such that, if we choose $\mathrm{P}_{\mathrm{n}}>0$, the recurrence relation (312) gives

$$
\mathrm{P}_{\mathrm{n}-1}>0, \quad \mathrm{P}_{\mathrm{n}-1}-\mathrm{P}_{\mathrm{n}}>0
$$

The recurrence relation (312) may be written as

$$
\begin{aligned}
& \mathcal{C}_{\mathrm{k}-1}(s, \mathrm{k}, l, M)\left(\mathrm{P}_{\mathrm{k}-1}-\mathrm{P}_{\mathrm{k}}\right)=\mathcal{C}_{\mathrm{k}+1}(s, \mathrm{k}, l, M)\left(\mathrm{P}_{\mathrm{k}}-\mathrm{P}_{\mathrm{k}+1}\right)- \\
& \left(C_{\mathrm{k}-1}(s, \mathrm{k}, l, M)+C_{\mathrm{k}}(s, \mathrm{k}, l, M)+C_{\mathrm{k}+1}(s, \mathrm{k}, l, M)\right) \mathrm{P}_{\mathrm{k}} .
\end{aligned}
$$

What makes the proof given by ${ }^{26}$ tick is the structure of the coefficients $\mathcal{C}_{\mathrm{k}-1}(s, \mathrm{k}, l, M)$, $\mathcal{C}_{\mathrm{k}}(s, \mathrm{k}, l, M)$, and $\mathcal{C}_{\mathrm{k}+1}(s, \mathrm{k}, l, M)$. In particular, the coefficients $\mathcal{C}_{\mathrm{k}-1}(s, \mathrm{k}, l, M), \mathcal{C}_{\mathrm{k}}(s, \mathrm{k}, l, M)$, and $\mathcal{C}_{\mathrm{k}+1}(s, \mathrm{k}, l, M)$ are such that, when $M=\frac{1}{2}$, the following relations hold

$$
\begin{aligned}
& \mathcal{C}_{\mathrm{k}-1}(s, \mathrm{k}, l, M)>0, \quad \mathcal{C}_{\mathrm{k}+1}(s, \mathrm{k}, l, M)>0 \\
& -\left(C_{\mathrm{k}-1}(s, \mathrm{k}, l, M)+C_{\mathrm{k}}(s, \mathrm{k}, l, M)+C_{\mathrm{k}+1}(s, \mathrm{k}, l, M)\right)>0
\end{aligned}
$$

for every $s, \mathrm{k}$, and $l$.

Equations (313), (314), (315), and (316), imply

$$
\mathrm{P}_{\mathrm{n}-2}-\mathrm{P}_{\mathrm{n}-1}>0, \quad \mathrm{P}_{\mathrm{n}-2}>0
$$

It follows by induction from equations (313), (314), (315), (316), and (317), that

$$
\mathrm{P}_{\mathrm{k}-1}-\mathrm{P}_{\mathrm{k}}>0, \quad \mathrm{P}_{\mathrm{k}-1}>0
$$

for all $\mathrm{k}=\mathrm{n}, \mathrm{n}-1, \mathrm{n}-2, \ldots, 1$.

When $\mathrm{k}=1$ equation (318) reads

$$
\mathrm{P}_{0}-\mathrm{P}_{1}>0, \quad \mathrm{P}_{0}>0
$$

Moreover, when $\mathrm{k}=0$ the three-term recurrence relation (312) reads

$$
\mathcal{C}_{0}(s, 0, l, M) \mathrm{P}_{0}+\mathcal{C}_{1}(s, 0, l, M) \mathrm{P}_{1}=0
$$


We arrive at a contradiction, because equations (319) and (320) are incompatible: Equation (319), and in particular the inequality $\mathrm{P}_{0}-\mathrm{P}_{1}>0$, implies that

$$
\mathcal{C}_{0}(s, 0, l, M) \mathrm{P}_{0}+\mathcal{C}_{1}(s, 0, l, M) \mathrm{P}_{1}>0
$$

This completes the proof given by C.H. ${ }^{26}$.

This proof fails when we try to apply it to the remaining families G3, E3, E7, and S3, which arise when $n=1$, in our consideration of the problem. The reason is that in our consideration of the problem, for all the remaining families G3, E3, E7, and S3, equation (315) holds for every $s, \mathrm{k}, l$, and $M$, but equation (316) does not hold for any of the remaining families G3, E3, E', and S3. For all the remaining families G3, E3, E7, and S3, and for every value of $M$, the sign of $-\left(C_{\mathrm{k}-1}(s, \mathrm{k}, l, M)+C_{\mathrm{k}}(s, \mathrm{k}, l, M)+C_{\mathrm{k}+1}(s, \mathrm{k}, l, M)\right)$ depends on $s, \mathrm{k}$, and $\mathrm{l}$. Consequently, in our consideration of the problem, we cannot use, for any of the remaining families G3, E3, EY, and S3, equation (314) in order to derive from it the sign of the difference $\mathrm{P}_{\mathrm{k}-1}-\mathrm{P}_{\mathrm{k}}$ from the signs of $\mathrm{P}_{\mathrm{k}}-\mathrm{P}_{\mathrm{k}+1}$ and $\mathrm{P}_{\mathrm{k}}$.

\section{G. Comments on the implementation of Kovacic's algorithm}

Kovacic's algorithm is based in the study of the structural properties of the Galois group of the differential equation $y^{\prime \prime}=\nu y, \nu \in C(x)$ (equation (20) ). Our results and the results of C.H. $\stackrel{26}{ }$ suggest that the implementation of Kovacic's algorithm can become more efficient when it is properly combined with the search for other types of symmetry.

In our consideration of the problem, in subsection VIIIB, we showed by using the elementary symmetries of the confluent Heun equation, that the examination of the two cases E3 and E7 can be reduced into the examination of the case E7 only (Corollary 3).

For every Fuchsian differential equation with $\mathrm{n}$ singular points and its various confluent forms there is a finite group of transformations which acts on the parameter space of the equation and generates alternative expressions for its family of local solutions. This group is of order $n ! 2^{\mathrm{n}-1}$ for every Fuchsian differential equation with $\mathrm{n}$ singular points.

In particular, for the hypergeometric equation this group is of order 24, and for the Heun's equation is of order 192 (see e.g. ${ }^{81}$ ). Moreover, for the confluent Heun equation this group is of order $16 \frac{78}{}$. It is precisely this group of elementary symmetries we use in subsection VIIIB in order to reduce the examination of the two cases E3 and E7 into the examination 
of the case E7 only. This group, in any case, can be derived in a systematic fashion with the method expounded e.g. in 11 .

A key observation regarding the work of C.H. is that at the very beginning of their study they use the transformation (14), which leaves equation $\mathcal{P} \mathcal{E}$ quasi-invariant. In principle a proof, such that given by C.H. ${ }^{26}$, can be given for one single value of $M$, or for a range of values of $M$. This is allowed precisely by the quasi-invariance of the differential equation $\mathcal{P E}$ under the transformation (14).

In general, if not in the problem under consideration, transformations of the form (14) can be of crucial importance when we study retained families, and examine if they give rise to Liouvillian solutions. C.H. do not explain how the transformation (14) can be derived from first principles. What is not well known, and is of outmost importance for the implementation of Kovacic's algorithm, is that such transformations can be derived in a systematic way ${ }^{82}$ by using the theory of Lie groups as this is applied to differential equations (see e.g. ${ }^{83}$ ).

Proving that a retained family does not give rise to a Liouvillian solution is one of the most difficult problems we have to solve when we apply Kovacic's algorithm. This in fact is an intriguing and difficult problem, it can be cast into different forms, and it can be approached with a variety of solution methods. Four such methods are the methods 1, 2, 3, and 4 , given in subsection VIIID.

Another method is the one we employ in subsection VIIIC, This method is in general applicable not only to second order ODEs, which arise when $n=1$, but it is also applicable to ODEs of any order, and in particular it is applicable to ODEs of order $n+1$ which arise when $\mathrm{n}=2,4,6$, and 12 .

A concrete realization of the method 3 given in subsection VIIID is the method of proof given by C.H. ${ }^{26}$. This method is in general applicable not only to three-term recurrence relations, but it is also applicable to higher order recurrence relations which may well arise when $\mathrm{n}=2,4,6$, and 12 , if not when $\mathrm{n}=1$.

The previous remarks suggest that the implementation of Kovacic's algorithm becomes more efficient when we follow the next steps

1. By using the theory of Lie groups as this is applied to differential equations, we search for transformations of the form (14) which leave equation $y^{\prime \prime}=\nu y, \nu \in C(x)$, quasi-invariant. In general equation $y^{\prime \prime}=\nu y, \nu \in C(x)$, may contain accessory parameters. In the case of the perturbation theory of black hole geometries such 
accessory parameters are the mass $M$, the charge $Q$, and the angular momentum $J$ of the black hole. In this case, if such a group of transformations which leave equation $y^{\prime \prime}=\nu y, \quad \nu \in C(x)$, quasi-invariant does exist, then equation $y^{\prime \prime}=\nu y$ will retain its form under this group of transformations, whereas $M, Q$, and $J$, will be replaced respectively by $\mathcal{M}, \mathcal{Q}$, and $\mathcal{J}$, where

$$
\begin{aligned}
\mathcal{M} & =f_{\mathcal{M}}(M, Q, J), \\
\mathcal{Q} & =f_{\mathcal{Q}}(M, Q, J), \\
\mathcal{J} & =f_{\mathcal{J}}(M, Q, J) .
\end{aligned}
$$

2. We apply Kovacic's algorithm to equation $y^{\prime \prime}=\nu y, \nu \in C(x)$.

3. Every remaining family, which in principle could give rise to a Liouvillian solution is associated to an ODE, which for example when $n=1$ is of second order and has the form $\mathrm{P}^{\prime \prime}+2 \theta \mathrm{P}^{\prime}+\left(\theta^{2}+\theta^{\prime}-\nu\right) \mathrm{P}=0$ (equation (73) $)$. If this ODE, e.g. if equation (73) , has a polynomial solution then a Liouvillian solution arises to equation $y^{\prime \prime}=\nu y, \nu \in C(x)$. For every such ODE we find a polynomial solution, or else we prove that it does not admit a polynomial solution.

4. When $\mathrm{n}=1$ each family is associated with an ODE which has the form $\mathrm{P}^{\prime \prime}+2 \theta \mathrm{P}^{\prime}+$ $\left(\theta^{2}+\theta^{\prime}-\nu\right) \mathrm{P}=0$ (equation (73) $)$. When $\mathrm{n}=2$ each family is associated with an ODE which has the form $\mathrm{P}^{\prime \prime \prime}+3 \theta \mathrm{P}^{\prime \prime}+\left(3 \theta^{2}+3 \theta^{\prime}-4 \nu\right) \mathrm{P}^{\prime}+\left(\theta^{\prime \prime}+3 \theta \theta^{\prime}+\theta^{3}-4 \nu \theta-2 \nu^{\prime}\right) \mathrm{P}=0$ (as we easily find from equation (44) ). Similarly, from equation (44), we find the form of ODEs associated with the families which arise when $n=4,6$, and 12 . For each such ODE we find the finite group of transformations which acts on the parameter space of the equation and generates alternative expressions for its family of local solutions. By using this group we can reduce the number of remaining families which could give rise to Liouvillian solutions to equation $y^{\prime \prime}=\nu y, \nu \in C(x)$. In the problem we consider in this paper it is precisely this finite group of symmetries of the confluent Heun equation, of order 16, we use in subsection VIIIB in order to reduce the examination of the two families E3 and E7 into the examination of the family E7 only.

5. Proving that a retained family does not give rise to a Liouvillian solution is one of the most difficult problems we have to solve when we apply Kovacic's algorithm, and it can 
be approached with a variety of solution methods. Four such methods are the methods $1,2,3$, and 4 , given in subsection VIIID. A concrete realization of the method 3 given in subsection VIIID is the method of proof given by C.H. $\underline{26}$.

6. This method of proof, as well as the method of proof we employ in subsection VIIIC and the methods of proof $1,2,3$, and 4 , we give in subsection VIIID, can be greatly facilitated if we take into account the group of transformations, when such a group exists, which leaves equation $y^{\prime \prime}=\nu y, \nu \in C(x)$ quasi-invariant, and if we use the subsequent relations (322), (323), and (324). This group of transformations, and the subsequent relations (322), (323), and (324) allow us to give such proofs for specific values, or for specific ranges of values, of the parameters $M, Q$, and $J$. Even more, in general, we expect that some of these proofs cannot be given if we do not employ the quasi-invariance of $y^{\prime \prime}=\nu y, \nu \in C(x)$, and the subsequent relations (322), (323), and (324).

7. In the search for polynomial solutions to the ODEs associated to the various values of $\mathrm{n}$, and in particular in the search for Liouvillian pairs $\eta$ and $\eta \int \frac{e^{-\int a}}{\eta^{2}}$, we can also employ the method of proof given in subsection VC.

8. Furthermore, in the search for polynomial solutions to the ODEs associated to the various values of $\mathrm{n}$, both in other black hole geometries, 4-dim and higher $\underline{\underline{41}}$, and in every other application of Kovacic's algorithm, we examine, if under certain conditions (corresponding to the $c=j$ ( $=0,1,2, \ldots$, fixed) condition in the Schwarzschild case) the square matrix $\mathcal{M}$ of the linear homogeneous system associated to these ODEs takes the form $\mathcal{M}=\left(\begin{array}{ll}\mathcal{A} & \mathrm{O} \\ \mathcal{D} & \mathcal{B}\end{array}\right)$. If this is indeed the case then this will be an indication that a generalization of Hautot's analysis can be applied to these ODEs, generalization similar to the generalization given in subsections VIIB, VIIC and VIID, As a result of this analysis, the polynomials contained in the derived Liouvillian solutions, will be written as finite sums of special functions of Mathematical Physics. We elaborate more on this and related issues in section [X] which follows.

To the best of our knowledge, in the applications of Kovacic's algorithm so far, the steps $1,4,6,7$, and 8 which greatly facilitate its thorough and complete implementation, have not been employed. 


\section{DISCUSSION}

Regarding the results we obtained and the related open questions and problems the following remarks are now in order:

1. Our results are apt to generalization to other black hole geometries, 4-dim and higher $\underline{41}$. It is an interesting open problem to apply Kovacic's algorithm to the master equation which governs the evolution of first order perturbations of other black hole geometries, 4-dim and higher $\underline{41}$, identify Liouvillian pairs, if they exist at all, as we did in section $\nabla$, find the polynomial solutions to the differential equations (44) associated to the Liouvillian solutions in "closed form", as we did in subsection VC, and express them as a finite sum of special functions of Mathematical Physics as we did in subsections VIIC and VIID.

2. As we proved in sections $\mathrm{V}$ and VIII in the case of the perturbations of the Schwarzschild geometry, the differential equation (44) associated to the Liouvillian solutions turns out to be the confluent Heun equation (144). In the case of other black hole geometries, 4-dim and higher $\stackrel{41}{ }$, equation (44) will turn out to be, in general, other type of equation, even of third or higher order.

3. Also, it will be interesting to examine, if in the case of other black hole geometries, Hautot's results can be generalized, and extended, if necessary, as they were extended in the case of Schwarzschild geometry in subsections VIIB, VIIC and VIID, so that the polynomials contained in the corresponding Liouvillian solutions can be written as a finite sum of special functions of Mathematical Physics.

4. The crucial starting remark made by Hautot upon which his whole analysis is based is that if $c=j(=0,1,2, \ldots$, fixed $)$ in $z(z-1) \mathrm{P}^{\prime \prime}(z)+\left(a z^{2}+b z+c\right) \mathrm{P}^{\prime}(z)+(d+$ $\left.e z+f z^{2}\right) \mathrm{P}(z)=0$ (equation (121) $)$, then the square matrix $\mathcal{M}$ of the associated linear homogeneous system takes the form $\mathcal{M}=\left(\begin{array}{ll}\mathcal{A} & \mathrm{O} \\ \mathcal{D} & \mathcal{B}\end{array}\right)$, and consequently (equation (132) )

$$
\operatorname{det}(\mathcal{M})=\operatorname{det}(\mathcal{A}) \cdot \operatorname{det}(\mathcal{B})
$$

$\operatorname{det}(\mathcal{A})=0$ becomes one of Hautot's sufficient conditions (equation (136) $)$. 
5. Therefore, the starting point in the generalization of Hautot's results to other black hole geometries, 4-dim and higher $\stackrel{41}{ }$, will be to examine, if under certain conditions (corresponding to the $c=j(=0,1,2, \ldots$, fixed) condition in the Schwarzschild case) the square matrix $\mathcal{M}$ of the linear homogeneous system associated to the differential equation (73) takes the form $\mathcal{M}=\left(\begin{array}{ll}\mathcal{A} & \mathrm{O} \\ \mathcal{D} & \mathcal{B}\end{array}\right)$.

6. In the case of the gravitational perturbations of the Schwarzschild geometry, and in particular in case G7 of Kovacic's algorithm, Hautot's sufficient condition $\operatorname{det}(\mathcal{A})=0$ is equivalent to condition (216), i.e., to the condition that the frequency $s$ of the perturbations takes the values $s=\frac{l(l-1)(l+1)(l+2)}{6}, l=2,3, \ldots$, of the frequencies of the algebraically special perturbations ${ }^{14}, 16$ of the Schwarzschild geometry!

7. As we proved in subsections VIIB, VIIC andVIID, in the case G7 of the gravitational perturbations of the Schwarzschild geometry, which is the case of Chandrasekhar's Liouvillian solution $\chi \int \frac{\mathrm{d} r_{*}}{\chi^{2}}$, studied in subsection $\mathrm{VC}$, when $s=\frac{l(l-1)(l+1)(l+2)}{6}, l=$ $2,3, \ldots$, the differential equation (73), which is the confluent Heun equation, an equation with three singular points, admits polynomial solution which can be written as a finite sum of truncated confluent hypergeometric functions of the first kind, and also, as a sum of associated Laguerre polynomials; both the truncated confluent hypergeometric functions of the first kind, and the associated Laguerre polynomials satisfy differential equations with two singular points! So, it appears, as Hautot remarks that one singular point disappears!

8. The most important aspect in Hautot's work ${ }^{60}-\underline{63}$ is his proof that certain ODEs of second order with polynomial coefficients and three singular points, when certain necessary conditions are satisfied, admit polynomial solutions which can be expressed as a finite sum of polynomials which satisfy differential equations with two singular points. On the first sight it appears that this is a purely "mathematical" fact which does not have any "physical" underpinnings, and/or connections with problems coming from Physics.

9. One of the most important spin-offs of the work we present in this paper is that Hautot's sufficient conditions, in the case of the gravitational perturbations of the 
Schwarzschild geometry, are nothing but the condition that the frequency of the perturbations takes the values of the frequencies of the algebraically special perturbations $\underline{14}, \underline{16}$ of the Schwarzschild geometry. There is no reason whatsoever that we could have anticipated this result.

10. This suggests that, at least in the case of the gravitational perturbations of the Schwarzschild geometry, Hautot's remarkable result, when certain sufficient conditions are satisfied a singularity of an ODE be removed as a method of solving this differential equation, is inextricably interwoven with the existence of algebraically special gravitational perturbations in this geometry. Thus it is plausible to conjecture that the removal of the singularity has a "physical origin".

11. Thus the "physical origin", if it exists, is expected to be related to the gravitational algebraically special perturbations of the Schwarzschild geometry. A natural measure of the complexity of a (confluent) Fuchsian equation is the number of its singular points. This suggests that a possible candidate for the "physical origin" of the removal of the singularity in the confluent Heun equation (144) is the extremum of the correction to the entropy of the Schwarzschild black hole due to the presence of a gravitational algebraically special perturbation. Needless to say this is highly speculative.

12. The extremum is understood with respect to a general, not algebraically special, gravitational perturbation of the Schwarzschild geometry, and/or respect with other type of perturbations of the Schwarzschild geometry. The correction to the entropy of the Schwarzschild black hole due to the presence of gravitational algebraically special perturbations, or due to the presence of other type of perturbations, can be calculated with the brick wall model of 't Hooft (see e.g. ${ }^{.84}$ ).

13. Hautot makes his crucial observation about the confluent Heun equation, namely, when certain sufficient conditions are satisfied, the confluent Heun equation admits polynomial solution which can be expressed as a sum of truncated confluent hypergeometric functions of the first kind in ${ }^{60}$. Moreover, as we clarified in subsection VID 2 the aforementioned polynomial solution can also be expressed as a sum of associated Laguerre polynomials. The important point, as Hautot points out $\underline{60}$ is that the confluent Heun equation has three singular points, whereas, hypergeometric functions (and 
Laguerre polynomials) satisfy differential equations with two singular points. So it seems that a singular point has disappeared!

14. Hautot in a series of papers $\frac{61}{\underline{63}}$ gives other confluent Fuchsian equations of second order, with three singular points, where the same phenomenon occurs: They admit polynomial solutions which are linear combinations of polynomials which satisfy confluent Fuchsian equations of second order with two singular points. However, what is of outmost significance for our study, is that Hautot does not give an explanation of the phenomenon. This phenomenon, is important on its own right, and irrespective of any possible "physical origin" it might have, it needs to be explained mathematically.

15. Not surprisingly, this phenomenon, after its initial observation by Hautot $\underline{60}$, attracted further attention, and to the best of our knowledge, the most complete and thorough study with a mathematical explanation of it, was given by Craster and Shanin in $\underline{\underline{64}}$ (see also $\frac{85}{}$ for a subsequent study based on $\left.{ }^{64}\right)$. It is somehow surprising that it took thirty years before a systematic theory about Hautot's observation could be developed.

16. The key observation in ${ }^{64}$ is that a (confluent) Fuchsian ODE of second order may have false singular points; i.e., singular points which are singular points of the coefficients of the ODE but not of the solutions of the ODE. This suggests that when a (confluent) Fuchsian ODE has false singular points it may have solutions which are expressed as linear combinations of functions which solve ODEs with coefficients which do not have the false singular points as singular points, and in this sense they are simpler than the initial ODE.

17. $\operatorname{In}^{64}$ it is proved that this is indeed the case when the false singular points have roots of the associated indicial equations 0 and $N$, where $N$ is a positive integer, and the local expansions around these false singular points contain no logarithmic terms in their developments. Let us call these false singular points of type $\mathcal{A}$. In fact Shanin and Craster prove that false singular points of type $\mathcal{A}$ can be removed by using isomonodromy mappings of the solution space of the ODE and show that the same isomonodromy mappings can be used in order to find solutions of the ODE which are expressed as linear combinations of functions which solve simpler ODEs which do not have in their coefficients the false singular points of type $\mathcal{A}$ as singular points. 
18. Interestingly enough the singular point 1 of the confluent Heun equation (144) is a false singular point of type $\mathcal{A}$. Even more what lies behind Hautot's observation $\underline{60} \underline{-63}$ is the theory developed by Shanin and Craster in ${ }^{64}$. It will be interesting to derive Hautot's results, and in particular expansion (154) with coefficients $A_{k}$ given by the linear homogeneous system (312), when condition (160) is satisfied, by using the theory developed in $\underline{64}$. Moreover, it will be interesting to investigate if the extension of Hautot's results, given in subsections VIIB, VIIC and VIID, can be derived by using the theory developed in $\frac{64}{4}$, or if an extension and/or modification of this theory is needed, in order to derive the extension of subsections VIIB, VIIC and VIID, by using this theory.

19. Hautot's results, in conjunction with the underlying theory developed in $\underline{64}$, provide the means in order to address a whole new area of research pertaining to black hole physics and to the theory of solvability of ODEs. The objective of the part of research related to black hole physics will be to find solutions in quadratures of the first order perturbation equations of the black hole geometries in 4-dim and higher $\underline{41}$. The aim of the part of research related to the theory of solvability of ODEs will be to find solutions of (confluent) Fuchsian ODEs by removing false singular points from these equations and explore the connections with the solvability theory of other types of equations.

20. Guiding problems in the part of this research which pertains to black hole physics are:

(a) The application of Kovacic's algorithm to the first order perturbation equations of black hole space-times, 4-dim and higher, in order to find all solutions in quadratures of these equations.

(b) The application of Hautot's observation, in the way indicated in the remarks 4 and 5 in this section, with the simultaneous application of the theory developed $\mathrm{in}^{64}$, in order to solve the associated ODE, the associated ODE in the problem under consideration is the ODE given in equation (73), in each case.

Verifying that the theory in $\underline{\underline{64}}$ gives Hautot's results $\underline{60} \underline{\underline{63}}$ is the first natural step for the materialization of this research program. 
21. Guiding problems in the part of this research which pertains to the solvability theory of ODEs are:

(a) The extension of the theory developed by Shanin and Craster in $\underline{\underline{64}}$ to (confluent) Fuchsian equations of third order and higher. Shanin and Craster developed their theory for second order (confluent) Fuchsian equations.

(b) The study of the connections of the solvability theory of (confluent) Fuchsian equations of second order developed in $\underline{\underline{64}}$ with the solvability theory of other type of equations, e.g., with the solvability theory of Painlevé equations, and also, as Shanin and Craster point out $\underline{\underline{64}}$, with generalizations of the Darboux-Halphen system.

22. A different mathematical explanation of Hautot's observation can be given with the use of group theory, and in particular with the use of tridiagonalization ${ }^{65}$. Tridiagonalization is a cogent algebraic framework via which the Heun differential operator, and two of its confluent forms, the confluent Heun operator and the double confluent Heun operator can be obtained from the hypergeometric operator, the confluent hypergeometric operator, and the Hermite operator respectively.

23. It will be interesting to derive Hautot's results, and in particular expansion (154) with coefficients $A_{k}$ given by the linear homogeneous system (312), when condition (160) is satisfied, by using the algebraic framework of tridiagonalization $\underline{65}$. Moreover, it will be interesting to investigate if the extension of Hautot's results, given in subsections VIIB, VIIC and VIID, can be derived by using tridiagonalization, or if an extension and/or modification of this algebraic framework is needed in order to derive this extension.

24. The theory developed by Shanin and Craster in $\underline{64}$, is based on the notion of the false singular point of a (confluent) Fuchsian equation of second order, and uses isomonodromy mappings of its solution space in order to remove the false singular point from its coefficients, and derive solutions of this equation which are expressed as linear combinations of functions which solve simpler ODEs, i.e. ODEs which do not have the false singular point in their coefficients.

25. By doing so Shanin and Craster effectively construct more involved operators from simpler ones. They use the Heun equation, which is the more general Fuchsian equation 
of second order with four regular singular points to develop their theory. In the subsection 4.3 of their paper they also consider the confluent Heun equation. They effectively construct the Heun operator from the hypergeometric operator and the confluent Heun operator from the confluent hypergeometric operator.

26. On the other hand tridiagonalization constructs explicitly $\underline{65}$ more involved operators from simpler operators, and in particular, it constructs the Heun operator from the hypergeometric operator, the confluent Heun operator from the confluent hypergeometric operator, and the double confluent Heun operator from the Hermite operator. It will be interesting to compare the results of the two approaches, that of Shanin and Craster $\underline{\underline{64}}$ and that of tridiagonalization $\underline{\underline{65}}$, and see if their results coincide in the cases of the Heun operator, of the confluent Heun operator, and of the double confluent Heun operator.

27. Moreover, it will be interesting to examine the importance of the notion of the false singular point in tridiagonalization, and also it will be interesting to investigate how the two approaches complement each other and if they can merge in a coherent framework. Hautot's results $\underline{60} \underline{\underline{63}}$ and their extension, given in subsections VIIB, VIIC and VIID, provide guidance and a test bed for this investigation.

28. It is an open problem to find the whole set of polynomial solutions to the confluent Heun equation; some partial results have appeared in the literature $\underline{48,49}, \underline{60} \underline{63}$ but the whole set of polynomial solutions to this equation is missing. Hautot's results and their extension, given in subsections VIIB, VIIC and VIID, give only a subclass of the polynomial solutions to the confluent Heun equation, those solutions which can be expressed as a finite sum of truncated confluent hypergeometric functions of the first kind, and also, as a finite sum of associated Laguerre polynomials. 


\section{Appendix A: The case $n=2$}

Here the $n=2$ case is presented. Having set $n=2$ we go to step 2 .

\section{Second step:}

2a. It is not applicable since $n=2$.

2b. From equations (36), (49) it follows that

$$
\mathrm{E}_{0}=\{2-4 \sqrt{1-\beta}, 2,2+4 \sqrt{1-\beta}\} \cap Z
$$

Equation (A1) gives

$$
\begin{aligned}
& \text { For } \beta=-3 \quad \mathrm{E}_{0}=\{-6,2,10\} \\
& \text { For } \beta=0 \mathrm{E}_{0}=\{-2,2,6\} \\
& \text { For } \beta=1 \mathrm{E}_{0}=\{2\}
\end{aligned}
$$

For the second root $r=2$ we obtain

$$
\mathrm{E}_{2}=\{2-4 s, 2,2+4 s\} \cap Z .
$$

Equation (A5) implies

$$
\mathrm{E}_{2}=\{2-4 s, 2,2+4 s\} \quad \text { where } \quad s=\frac{\mathrm{k}}{4}-\frac{1}{2}, \mathrm{k} \in Z .
$$

2c. It is not applicable since $\mathrm{n}=2$.

2d. Since $\Gamma_{4}=\{\infty\}$ (see equation (47)), equation (41) implies

$$
\mathrm{E}_{\infty}=\{4\}
$$

\section{Third step:}

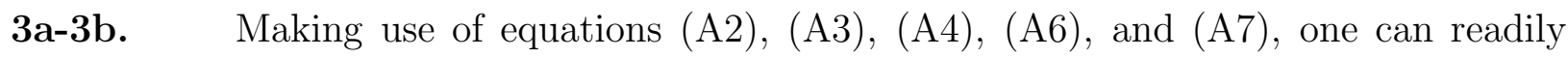
find the families $\underline{\mathrm{e}}=\left(\mathrm{e}_{c}\right)_{c \in \Gamma}$ of elements $\mathrm{e}_{c} \in \mathrm{E}_{c}$. Since $\mathrm{E}_{0}$ depends on the value of $\beta$ we distinguish three cases.

$$
\beta=-3 \quad \text { (Gravitational case) }
$$




\begin{tabular}{|c|c|c|}
\hline $\mathbf{e}_{0}$ & $\mathbf{e}_{2}$ & $\mathbf{e}_{\infty}$ \\
\hline-6 & $2-4 s$ & 4 \\
\hline-6 & 2 & 4 \\
\hline-6 & $2+4 s$ & 4 \\
\hline 2 & $2-4 s$ & 4 \\
\hline 2 & 2 & 4 \\
\hline 2 & $2+4 s$ & 4 \\
\hline 10 & $2-4 s$ & 4 \\
\hline 10 & 2 & 4 \\
\hline 10 & $2+4 s$ & 4 \\
\hline & (Scalar case) & \\
\hline $\mathbf{e}_{0}$ & $\mathbf{e}_{2}$ & $\mathbf{e}_{\infty}$ \\
\hline 2 & $2-4 s$ & 4 \\
\hline 2 & 2 & 4 \\
\hline 2 & $2+4 s$ & 4 \\
\hline & (Electromagnetic case) & \\
\hline $\mathbf{e}_{0}$ & $\mathbf{e}_{2}$ & $\mathbf{e}_{\infty}$ \\
\hline-2 & $2-4 s$ & 4 \\
\hline-2 & 2 & 4 \\
\hline-2 & $2+4 s$ & 4 \\
\hline 2 & $2-4 s$ & 4 \\
\hline 2 & 2 & 4 \\
\hline 2 & $2+4 s$ & 4 \\
\hline 6 & $2-4 s$ & 4 \\
\hline 6 & 2 & 4 \\
\hline 6 & $2+4 s$ & 4 \\
\hline
\end{tabular}


In none of these families one is to find two or more $\mathrm{e}_{c}$ which are odd numbers. Hence all of them are discarded.

Output:

CONTINUATION: It is not applicable since $\mathrm{n}=2$ is the largest element of $\mathrm{L}$.

OUTPUT2: The equation (9) does not have any Liouvillian solutions. 


\section{REFERENCES}

${ }^{1}$ Leaver E W 1986 Spectral decomposition of the perturbation response of the Schwarzschild geometry Phys. Rev. D. 34 384-407

${ }^{2}$ Regge T and Wheeler J A 1957 Stability of a Schwarzschild singularity Phys. Rev. 108 $1063-1069$

${ }^{3}$ Edelstein L A and Vishveshwara C V 1970 Differential Equations for Perturbations on the Schwarzschild Metric Phys. Rev. D. 1 3514-3517

${ }^{4}$ Vishveshwara C V 1970 Stability of the Schwarzschild Metric Phys. Rev. D. 1 2870-2879

${ }^{5}$ Chandrasekhar S and Detweiler S 1975 The quasinormal modes of the Schwarzschild black hole Proc. R. Soc. Lond. A. 344 441-452

${ }^{6}$ Leaver E W 1985 An analytic representation for the quasinormal modes of Kerr black holes Proc. R. Soc. Lond. A. 402 285-298

${ }^{7}$ Andersson N and Linnaus S 199 Quasinormal modes of a Schwarzschild black hole: Improved phase-integral treatment Phys. Rev. D. 46 4179-4187

${ }^{8}$ Nollert H P and Schmidt B G 1992 Quasinormal modes of Schwarzschild black hole: Defined and calculated via Laplace transformation Phys. Rev. D. 45 2617-2627

${ }^{9}$ Andersson N, Araújo M E and Schutz B F 1993 The phase-integral method and black hole normal modes Class. Quantum. Grav. 10 735-755

${ }^{10}$ Mashhoon B and Liu H 1996 On the spectrum of oscillations of a Schwarzschild black hole Class. Quantum. Grav. 13 233-251

${ }^{11}$ Onozawa H 1997 Detailed study of quasinormal frequencies of the Kerr black hole Phys. Rev. D. 55 3593-3602

12Zerilli F J 1970 Gravitational Field of a Particle Falling in a Schwarzschild Geometry Analysed in Tensor Harmonics Phys. Rev. D. 2 2141-2160

${ }^{13}$ Zerilli F J 1970 Effective potential for even-parity Regge-Wheeler gravitational perturbation equations Phys. Rev. Lett. 24, 737-738

${ }^{14}$ Chandrasekhar S 1984 On algebraically special perturbations of black holes Proc. R. Soc. Lond. A. 392 1-13

${ }^{15}$ Wald R M 1973 On perturbations of a Kerr black hole J. Math. Phys. 14 1453-1461

${ }^{16}$ Couch W E and Newman E T 1973 Algebraically secial perturbations of the Schwarzschild metric J. Math. Phys. 14285 
${ }^{17}$ Qi G and Schutz B 1993 Robinson-Trautman equations and Chandrasekhar's special perturbation of the Schwarzschild metric Gen. Rel. Gravit. 25 1185-1188

${ }^{18}$ Kovacic J 1986 An algorithm for solving second order linear homogeneous differential equations J. Symb. Comp. 2 3-43

${ }^{19}$ Duval A and Loday-Richaud M 1989 A propos de l'algotitme de Kovacic Tech. Rep. Universite de Paris-Sud Mathematiques Orsay France

${ }^{20}$ Duval A and Loday-Richaud M 1992 Kovacic's Algorithm and its Application to Some Families of Special Functions AAECC 3 211-246

${ }^{21}$ Ulmer F and Weil J A 1996 Note on Kovacic's algorithm J. Symbol. Comput. 22(2) $179-200$

${ }^{22}$ Singer M F and Ulmer F 1993 Liouvillian and algebraic solutions of second and third order linear differential equations J. Symbol. Comput. 16(1) 37-73

${ }^{23}$ Singer M F and Ulmer F 1995 Necessary conditions for Liouvillian solutions of (third order) linear differential equations $A A E C C \mathbf{6}(1) 1-22$

${ }^{24}$ van Hoeij M, Ragot J F, Ulmer F and Weil J A 1998 Liouvillian solutions of linear differential equations of order three and higher J. Symbol Comput. 28 589-609

${ }^{25}$ Melas E 2002 PhD Thesis University of London, QMW (unpublished)

${ }^{26}$ Couch W E and Holder C L 2007 Liouvillian perturbations of black holes J. Math. Phys. 48102502

${ }^{27}$ Couch W E 1981 Solutions to wave equations on black hole geometries J. Math. Phys. 22 $1457-1462$

${ }^{28}$ Couch W E 1985 Solutions to wave equations on black hole geometries. II J. Math. Phys. $262266-2296$

${ }^{29}$ Couch W E and Holder C L 2012 Solutions to wave equations on black hole geometries. II J. Math. Phys. 53062502

${ }^{30}$ Fiziev P P 2006 On the Exact Solutions of the Regge-Wheeler Equation in the Schwarzschild Black Hole Interior arXiv: 0603003v4

${ }^{31}$ Fiziev P P 2009 Classes of Exact Solutions to Regge-Wheeler and Teukolsky Equations arXiv: $0902.1277 \mathrm{v} 3$

${ }^{32}$ Borissov S R and Fiziev P P 2010 Exact Solutions of Teukolsky Master Equation with Continuous Spectrum arXiv: 0903.3617v3

${ }^{33}$ Lèa Jaccoud El-Jaick and Bartolomeu D B Figueiredo 2009 A limit of the confluent Heun 
equation and the Schrödinger equation for an inverted potential and for an electric dipole arXiv: 0902.3202v2

${ }^{34}$ Lèa Jaccoud El-Jaick and Bartolomeu D B Figueiredo 2011 ON CERTAIN SOLUTIONS FOR CONFLUENT AND DOUBLE-CONFLUENT HEUN EQUATIONS arXiv: $0807.2219 \mathrm{v} 3$

${ }^{35}$ Lèa Jaccoud El-Jaick and Bartolomeu D B Figueiredo 2011 Transformations of Heun's equation and its integral relations arXiv: 1002.4559v3

${ }^{36}$ Lèa Jaccoud El-Jaick and Bartolomeu D B Figueiredo 2012 Confluent and Double-Confluent Heun Equations: Convergence of Solutions in Series of Coulomb Wavefunctions arXiv: 1209.4673v1

${ }^{37}$ Lèa Jaccoud El-Jaick and Bartolomeu D B Figueiredo 2013 Integral relations for solutions of confluent Heun equations arXiv: 1311.3703v1

${ }^{38}$ Lèa Jaccoud El-Jaick and Bartolomeu D B Figueiredo 2013 New solutions to the confluent Heun equation and quasiexact solvability arXiv: $1311.7677 \mathrm{v} 1$

${ }^{39}$ Maasen A and Brink V D 2000 Analytic treatment of black-hole gravitational waves at the algebraically special frequency Phys. Rev. D 62064009

${ }^{40}$ Maciejewski A J, Strelcyn J-M and Szydlowski M 2001 Nonintegrability of Bianchi VIII Hamiltonian system J. Math. Phys. 42 1728-1743

${ }^{41}$ Ida D, Ishibashi A and Shiromizu T 2011 Topology and Uniqueness of Higher Dimensional Black Holes Progress of Theoretical Physics Supplement 189 52-92

${ }^{42}$ Landau L D and Lifshitz E M 1965 Quantum Mechanics Non-Relativistic Theory Oxford: Pergamon Press.

${ }^{43}$ Chee G 1994 Note on a solution of the algebraically special perturbation of the Schwarzschild black hole J. Math. Phys. 35 3025-3028. The author of this paper, misreading Chandrasekhar's article $\frac{14}{4}$, mistakenly concludes that the solution $\chi=\frac{\mu^{2} r+6}{r} \mathrm{e}^{-\mathrm{i} \sigma r_{*}}$ does not appear in the latter's paper. However, Chandrasekhar states (page 12 of his article): "The general (my italics) solution of equation (104) ... is given by (cf. equation (77)) $\mathrm{Z}^{( \pm)}=\chi \int \frac{\mathrm{d} r_{*}}{\chi^{2}}(107)$ ". Since an indefinite integral is defined up to an additive constant, the solution $\chi$ is included in the expression (107).

${ }^{44}$ Araujo M E and MacCallum M A H On the algebraically special perturbations of Schwarzschild black holes unpublished

${ }^{45}$ Gautschi W 1967 Computational aspects of three-term recurrence relations Siam Review 


\section{$924-82$}

${ }^{46}$ Gurappa N, Panigrahi P K and Shreecharan T 2003 A new perspective on single and multi-variate differential equations Journal of Computational and Applied Mathematics $160103-112$

${ }^{47}$ Gurappa N and Panigrahi P K 2004 On polynomial solutions of the Heun equation $J$. phys. A:Math. Gen. 37 L605-L608

${ }^{48}$ Ishkhanyan T A and Ishkhanyan A M Expansions of the solutions to the confluent Heun equation in terms of the Kummer confluent hypergeometric functions arXiv:1403.1833

${ }^{49}$ Ishkhanyan A 2005 Incomplete beta-functon expansions of the solutions to the confluent Heun equation J. phys. A: Math. Gen. 38 L491-L498

${ }^{50}$ Heun K 1889 Math. Ann. 33161

${ }^{51}$ Bateman H and Erd'elyi A 1955 Higher Transcendental Functions Vol. 3 (New York Toronto London: Mc Grow-Hill Comp. INC)

${ }^{52}$ Decarreau A, Dumont-Lepage M Cl, Maroni P, Robert A and Roneaux A 1978 Ann. Soc. Buxelles 9253

${ }^{53}$ Decarreau A, Maroni P and Robert A 1978 Ann. Soc. Buxelles 92151

${ }^{54}$ Heun's Differential Equations 1995 ed Roneaux A (Oxford: Oxford Univ. Press)

${ }^{55}$ Slavyanov S Y and Lay W Special Functions, A Unified Theory Based on Singularities 2000 (Oxford: Oxford Mathematical Monographs)

${ }^{56}$ Maier R S 2004 The 192 Solutions of Heun Equation arXiv: math/0408317

${ }^{57}$ Galtsov D V and Ershov A A 1989 Soviet Phys. Jour. 32 13-18

${ }^{58}$ Suziki H, Takasugi E and Umetsu H 1998 Progr. Theor. Phys. 100491

${ }^{59}$ Batic D and Schmid H 2007 JMP 48042502

${ }^{60}$ Hautot A 1969 Sur les solutions polynomiales de l' équation différentielle $z(z-1) P_{\mathrm{n}}^{\prime \prime}+$ $\left(a z^{2}+b z+c\right) P_{\mathrm{n}}^{\prime}+\left(d+e z+f z^{2}\right) P_{\mathrm{n}}^{\prime}=0$ Bulletin de la Sociètè Royale des Sciences de Liège 38 654-659

${ }^{61}$ Hautot A 1969 Sur les solutions polynomiales de l' équation différentielle $z P_{\mathrm{n}}^{\prime \prime}+\left(a z^{2}+\right.$ $b z+c) P_{\mathrm{n}}^{\prime}+\left(d+e z+f z^{2}\right) P_{\mathrm{n}}^{\prime}=0$ Bulletin de la Sociètè Royale des Sciences de Liège $\mathbf{3 8}$ 660-663

${ }^{62}$ Hautot A 1971 Sur les solutions polynomiales de l' équation différentielle $z(1-z)(a-$ $z) P_{\mathrm{n}}^{\prime \prime}+\left(a z^{2}+b z+c\right) P_{\mathrm{n}}^{\prime}+\left(d+e z+f z^{2}\right) P_{\mathrm{n}}^{\prime}=0$ Bulletin de la Sociètè Royale des Sciences de Liège 40 7-12 
${ }^{63}$ Hautot A 1971 Sur des combinaisons linéaires d'un nombre fini de fonctions transcendantes comme solutions d'équations différentielles du second ordre Bulletin de la Sociètè Royale des Sciences de Liège $\mathbf{4 0} 13-23$

${ }^{64}$ Shanin A V and Craster R V 2002 Removing false singular points as a method of solving ordinary differential equations Euro. Journal of Applied Mathematics 13 617-639

${ }^{65}$ Grünbaum F A, Vinet L and Zhedanov A Tridiagonalization and the Heun equation J. Math. Phys. 58, 031703 (2017); e-print arXiv:1602.04840v1

${ }^{66}$ Georgiev G N and Georgieva-Grosse M N 2005 Journal of Telecommunications and Information Technology 3112

${ }^{67}$ Ishkhanyan A M Schrödinger potentials solvable in terms of the general Heun functions arXiv:1601.03360

${ }^{68}$ Ishkhanyan A M 2016 Schrödinger potentials solvable in terms of the confluent Heun functions Theoretical and Mathematical Physics 188 980-993

${ }^{69}$ Ishkhanyan A M The third exactly solvable hypergeometric quantum-mechanical potential arXiv:1602.07685

${ }^{70}$ Dereziński J and Wrochna M Exactly solvable Schrödinger operators arXiv:1009.0541v2

${ }^{71}$ Chandrasekhar S 1975 On the equations governing the perturbations of the Schwarzschild black hole Proc. R. Soc. Lond. A. 343 289-298

${ }^{72}$ Chandrasekhar S 1983 The Mathematical Theory of black holes (New York: Oxford University Press)

${ }^{73}$ Wheeler J A 1955 Geons Phys. Rev. 97 511-536

${ }^{74}$ Ruffini R, Tiomno J and Vishveshwara C V 1972 Electromagnetic Field of a Particle Moving in a Spherically Symmetric Black-Hole Background Let. Al N. Cim. 3 211-215

${ }^{75}$ Price R H 1972 Nonspherical Perturbations of Relativistic Gravitational Collapse. I.Scalar and Gravitational Perturbations Phys. Rev. D. 5 2419-2438

${ }^{76}$ Bardeen J M and Press W H 1973 Radiation fields in the Schwarzschild background J. Math. Phys. 14 7-19

${ }^{77}$ Hanni R S and Ruffini R 1973 Lines of force of a point charge near a Schwarzschild black hole Black holes (Les Astres Occlus) page R57 DeWitt C and DeWitt B S (Editors) (Gordon and Breach Science Publishers)

${ }^{78}$ El-Jaick L J and Figueiredo B D B 2011 Transformations of Heun's equation and its integral relations J. Phys. A: Mathematical and Theoretical 44075204 
${ }^{79}$ Abramowitz A and Stegun I A (Eds.) 1964 Handbook of Mathematical Functions with Formulas, Graphs, and Mathematical Tables. National Bureau of Standards Applied Mathematics Series, U.S. Government Printing Office, Washington, D.C..

${ }^{80}$ Kazakov A Ya 2003 SYMMETRIES OF THE CONFLUENT HEUN EQUATION Journal of Mathematical Sciences 117 3918-3927

${ }^{81}$ Maier R S 2007 THE 192 SOLUTIONS OF THE HEUN EQUATION MATHEMATICS OF COMPUTATION $\mathbf{7 6} 811-843$

${ }^{82}$ Theodosios Christodoulakis (Private Communication)

${ }^{83}$ Olver P J Applications of Lie Groups to Differential Equations (New York: Springer)

${ }^{84}$ Ghosh K 2016 Near-horizon geometry and the entropy of a minimally coupled scalar field in the Kerr black hole Prog Theor Exp Phys 9 093E03

${ }^{85}$ Takemura K 2012 Heun's equation, generalized hypergeometric function and exceptional Jacobi polynomial Journal of Physics A: Mathematical and Theoretical 458085211 